University of Tennessee Health Science Center

UTHSC Digital Commons

\title{
$5-2013$
}

\section{A Series of Pharmaceutical Technology Concepts Applied to Pediatric Formulations}

Hao Lou

University of Tennessee Health Science Center

Follow this and additional works at: https://dc.uthsc.edu/dissertations

Part of the Medicinal and Pharmaceutical Chemistry Commons, and the Pharmaceutics and Drug Design Commons

\section{Recommended Citation}

Lou, Hao, "A Series of Pharmaceutical Technology Concepts Applied to Pediatric Formulations" (2013). Theses and Dissertations (ETD). Paper 153. http://dx.doi.org/10.21007/etd.cghs.2013.0190.

This Thesis is brought to you for free and open access by the College of Graduate Health Sciences at UTHSC Digital Commons. It has been accepted for inclusion in Theses and Dissertations (ETD) by an authorized administrator of UTHSC Digital Commons. For more information, please contact jwelch30@uthsc.edu. 


\title{
A Series of Pharmaceutical Technology Concepts Applied to Pediatric Formulations
}

\begin{abstract}
Pediatric formulations have received great attention from regulatory agencies in the United States and Europe. Since solid dosage forms such as tablets and capsules will not be able to improve pediatric patients' compliance, customized pediatric formulations are needed. In response, formulators in the pharmaceutical industry have made significant effort into developing various pediatric formulations. However, developing satisfactory pediatric formulations is a process that is guided by case by case basis. The objective of this current study was to apply the scientific pharmaceutical technology concepts into developing modern pediatric formulations. We have shown that the drug delivery design will be affected by the route of administration and by the type of dosage form utilized. We are hopeful that our approach will serve as a tool to assist formulations development. Three drug molecules and an anion were utilized in this work (Chlorpheniramine Maleate, Prednisone, Sulfamerazine, and lodide ion). Each drug molecule was utilized according to its physicochemical properties along with the pediatric patients' needs. As a result, several pediatric formulations were developed. Here is the list: taste masked orally disintegrating tablet, taste masked orally disintegrating film, taste masked mini-tablet, reconstituted nanocrystals and a transdermal microemulsion. We are hopeful that these dosage forms will serve as good examples for specialized pediatric formulations and will attract pharmaceutical industry to adapt some these delivery systems into their development strategy.
\end{abstract}

\section{Document Type}

Thesis

\section{Degree Name}

Master of Science (MS)

\section{Program}

Pharmaceutical Sciences

\section{Research Advisor}

Hassan Almoazen, Ph.D.

\section{Keywords}

Film; Formulation; Microemulsion; Nanocrystal; Pediatric; Tablet

\section{Subject Categories}

Medicinal and Pharmaceutical Chemistry | Medicine and Health Sciences | Pharmaceutics and Drug Design | Pharmacy and Pharmaceutical Sciences 


\title{
A SERIES OF PHARMACEUTICAL TECHNOLOGY CONCEPTS APPLIED TO PEDIATRIC FORMULATIONS
}

\author{
A Thesis \\ Presented for \\ The Graduate Studies Council \\ The University of Tennessee \\ Health Science Center
}

In Partial Fulfillment

Of the Requirements for the Degree

Master of Science

From The University of Tennessee

By

Hao Lou

May 2013 
Portions of Chapter 2 and 3 (C) 2013 by Informa Healthcare. Portions of Chapter 4 and 5 (c) 2013 by Springer.

All other material (C) 2013 by Hao Lou.

All rights reserved. 


\section{DEDICATION}

I dedicate this thesis to my parents, $\mathrm{Na}$ Shu and Xiaofeng Lou for their endless love and support. 


\section{ACKNOWLEDGEMENTS}

I would like to express my deepest gratitude to my research advisor, Dr. Hassan Almoazen, for his excellent guidance and training. I am also grateful to Dr. James Johnson, who gave me the support and the opportunity to work in "Tablet Technology" group. I would also like to thank the remaining members of my thesis committee:

Dr. Duane Miller, Dr. George Wood, and Dr. Gerald Rajan. Their valuable suggestions enabled me to accomplish this thesis.

I would also like to extend my special thanks to Mrs. Min Liu and Dr. Wen Qu, who genuinely helped me in developing some of these characterizing methods in this thesis. 


\begin{abstract}
Pediatric formulations have received great attention from regulatory agencies in the United States and Europe. Since solid dosage forms such as tablets and capsules will not be able to improve pediatric patients' compliance, customized pediatric formulations are needed. In response, formulators in the pharmaceutical industry have made significant effort into developing various pediatric formulations. However, developing satisfactory pediatric formulations is a process that is guided by case by case basis.

The objective of this current study was to apply the scientific pharmaceutical technology concepts into developing modern pediatric formulations. We have shown that the drug delivery design will be affected by the route of administration and by the type of dosage form utilized. We are hopeful that our approach will serve as a tool to assist formulations development. Three drug molecules and an anion were utilized in this work (Chlorpheniramine Maleate, Prednisone, Sulfamerazine, and Iodide ion). Each drug molecule was utilized according to its physicochemical properties along with the pediatric patients' needs. As a result, several pediatric formulations were developed. Here is the list: taste masked orally disintegrating tablet, taste masked orally disintegrating film, taste masked mini-tablet, reconstituted nanocrystals and a transdermal microemulsion. We are hopeful that these dosage forms will serve as good examples for specialized pediatric formulations and will attract pharmaceutical industry to adapt some these delivery systems into their development strategy.
\end{abstract}




\section{TABLE OF CONTENTS}

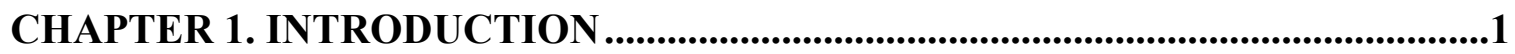

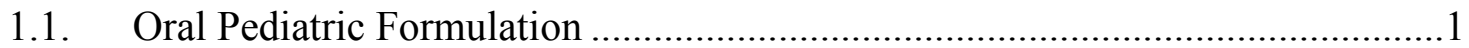

1.1.1. Orally disintegrating tablets (ODTs) .....................................................

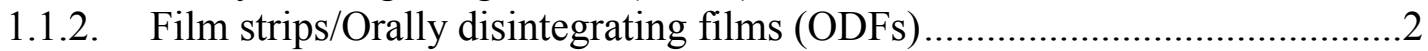

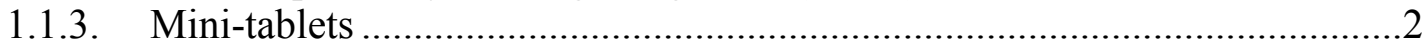

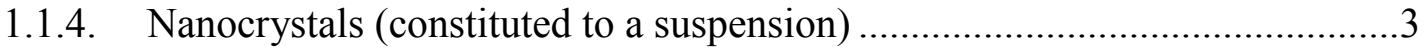

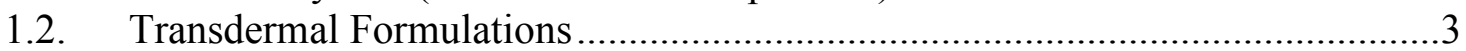

\section{CHAPTER 2. EVALUATION OF CHLORPHENIRAMINE MALEATE MICROPARTICLES IN ORALLY DISINTEGRATING FILM AND ORALLY

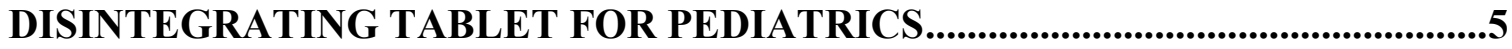

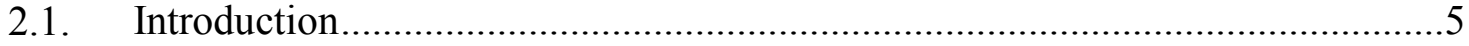

2.2. Experimental Procedures ..........................................................................6

2.2.1. Materials.............................................................................................. 6

2.2.2. Preparation of microparticles ..................................................................

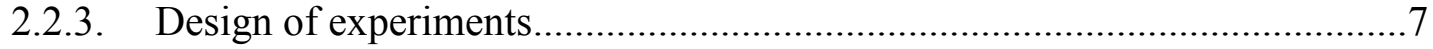

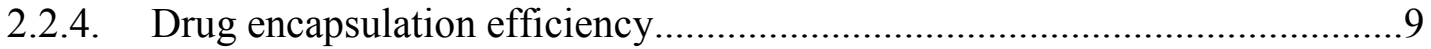

2.2.5. In vitro release testes .................................................................... 9

2.2.6. Checkpoint analysis .......................................................................... 9

2.2.7. Preparation of ODF and ODT ................................................................

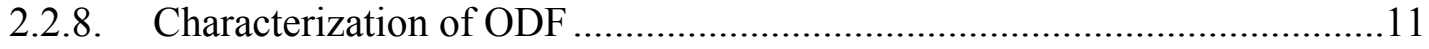

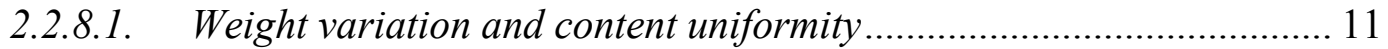

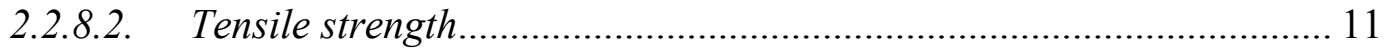

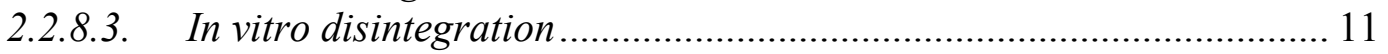

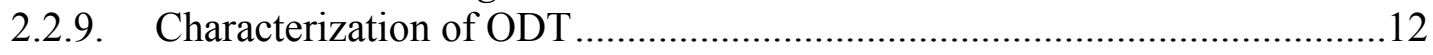

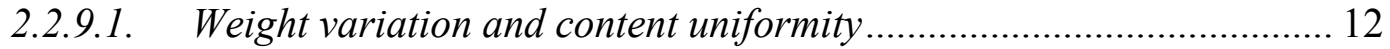

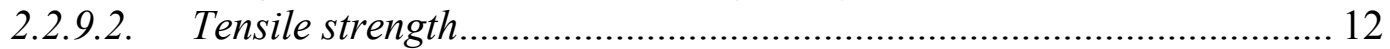

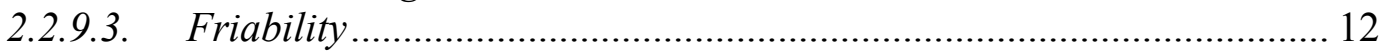

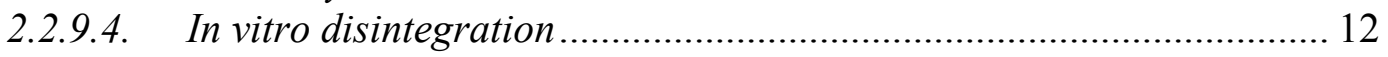

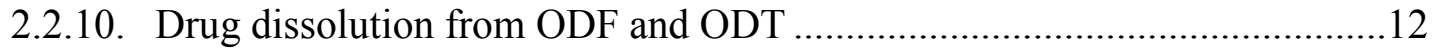

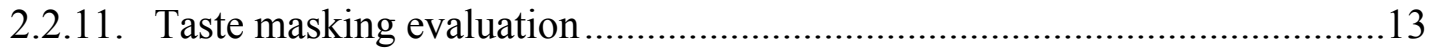

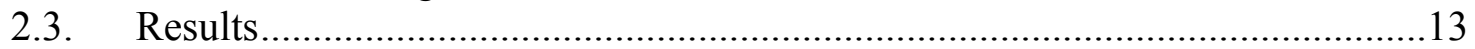

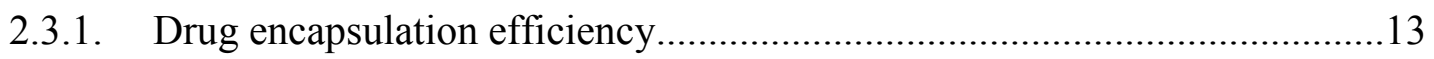

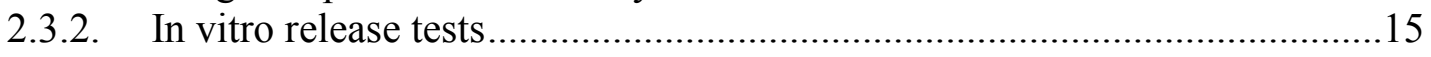

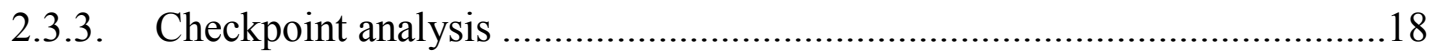

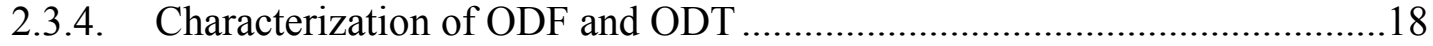

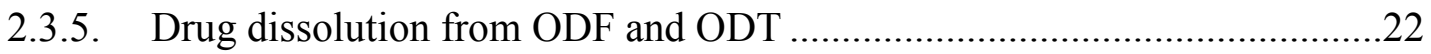

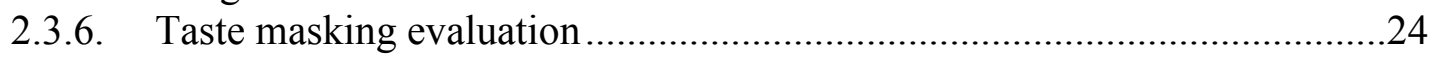

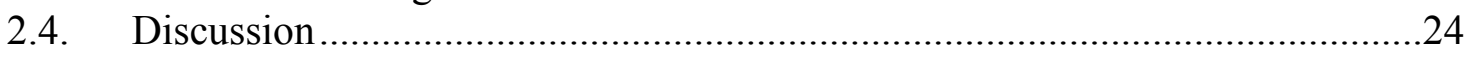

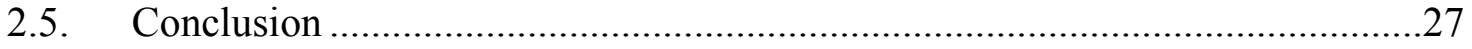




\section{CHAPTER 3. INFLUENCE OF SELECTED ANIONS ON MILLING OF SULFAMERAZINE NANOCRYSTAL}

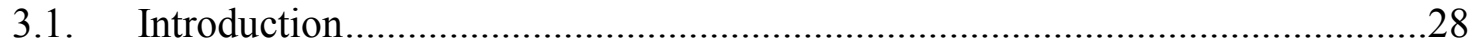

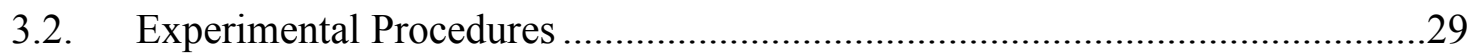

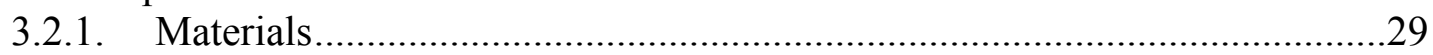

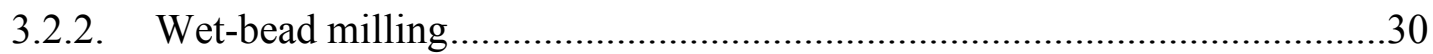

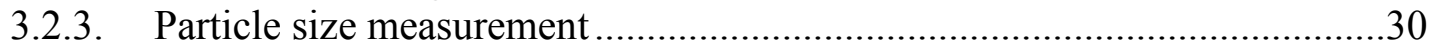

3.2.4. Determination of PVP adsorption on particle surface.................................30

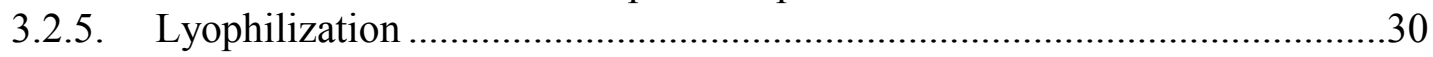

3.2.6. Determination of drug content in lyophilized powder blends..................... 31

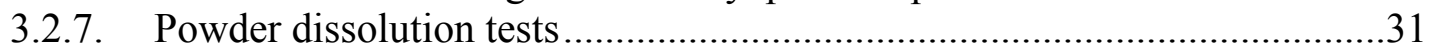

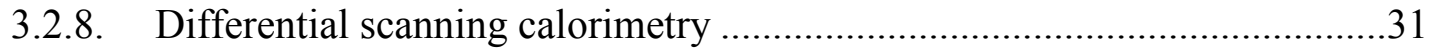

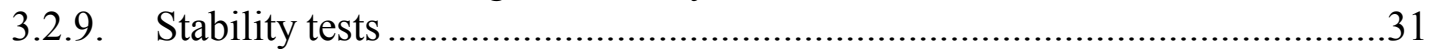

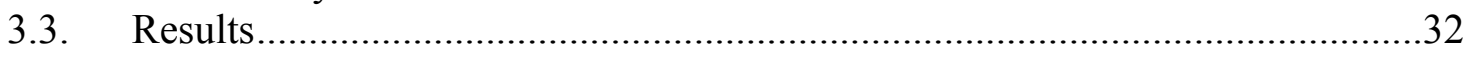

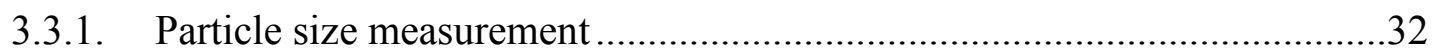

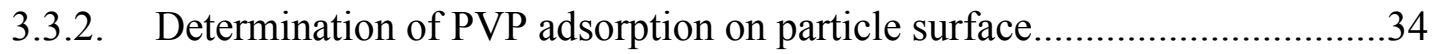

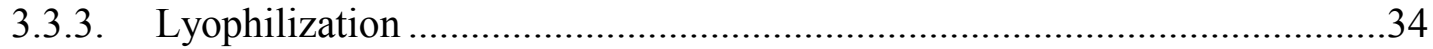

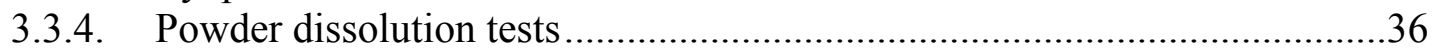

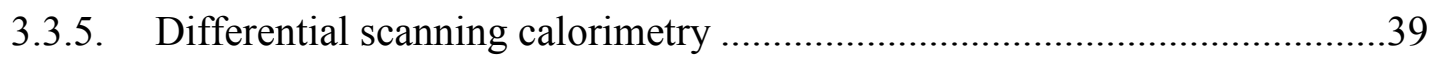

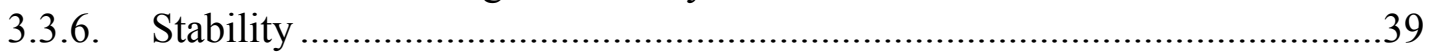

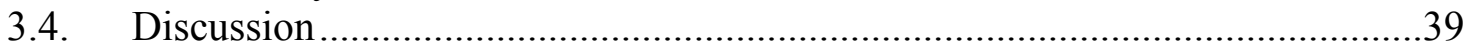

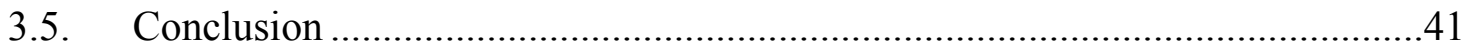

\section{CHAPTER 4. DEVELOPMENT OF A COATED MINI-TABLET OF CO- GRINDED PREDNISONE-NEUSILIN COMPLEX FOR PEDIATRIC USE .........42}

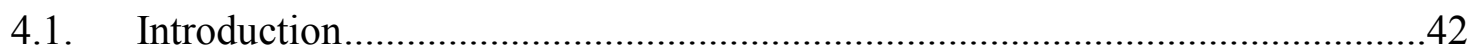

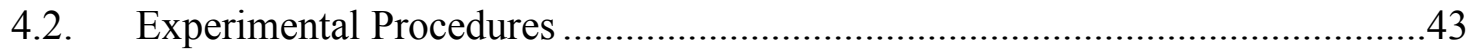

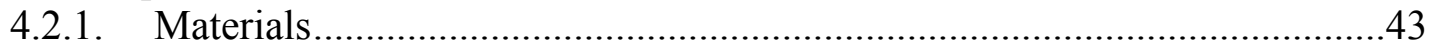

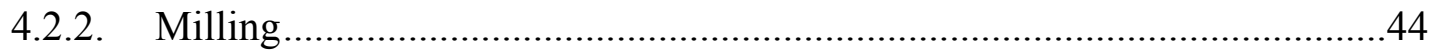

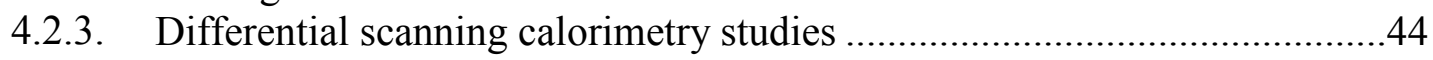

4.2.4. X-ray powder diffraction and percent crystallinity .................................44

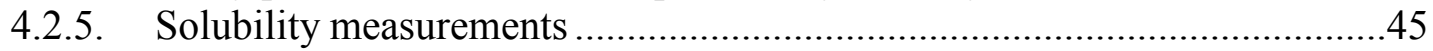

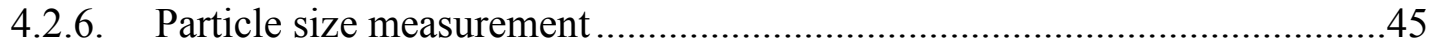

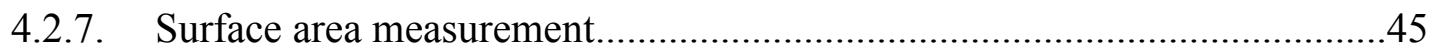

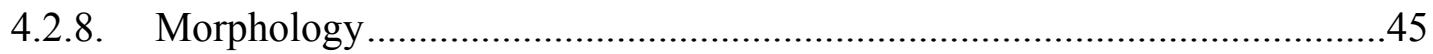

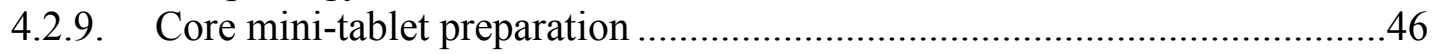

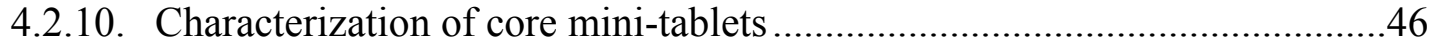

4.2.10.1. Thickness, weight variation and unit dose strength .......................... 46

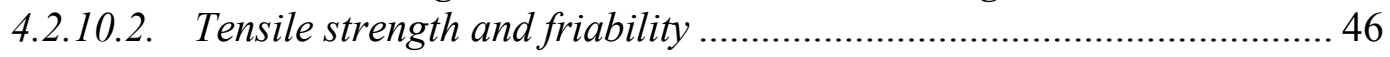

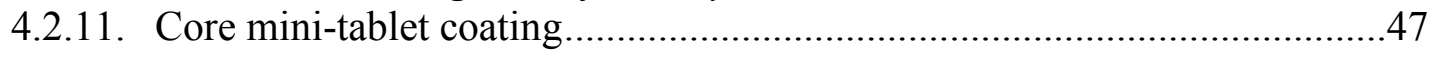

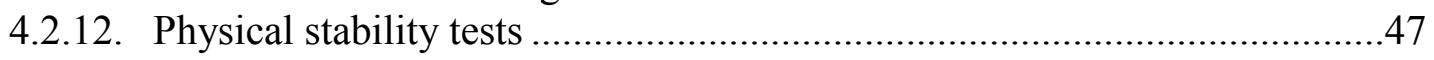

4.2.13. Powder and mini-tablet dissolution tests..............................................47

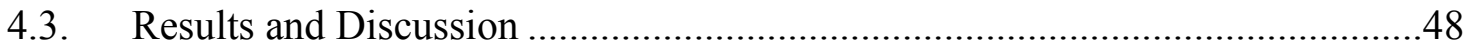

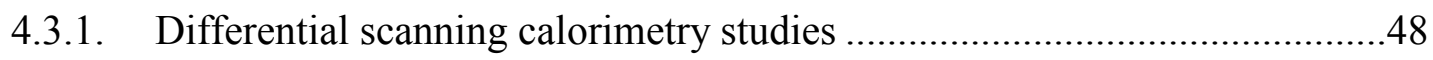

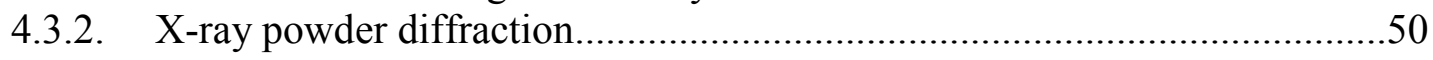

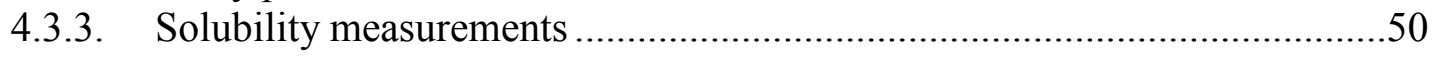


4.3.4. Surface area and particle size measurements ............................................53

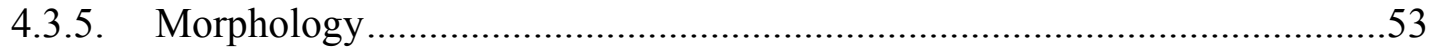

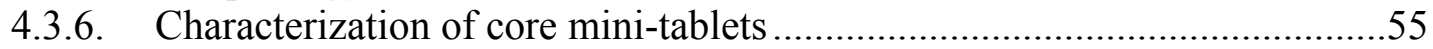

4.3.7. Powder and core mini-tablet dissolution profiles..........................................55

4.3.8. Coated mini-tablets dissolution profiles...................................................58

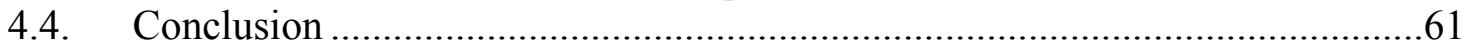

\section{CHAPTER 5. DEVELOPMENT OF W/O MICROEMULSION FOR TRANSDERMAL DELIVERY OF IODIDE IONS .....................................................64}

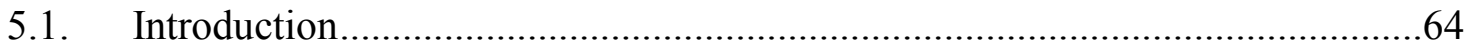

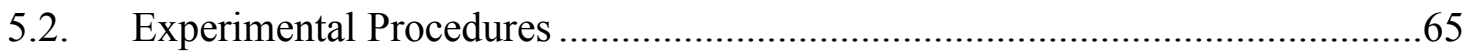

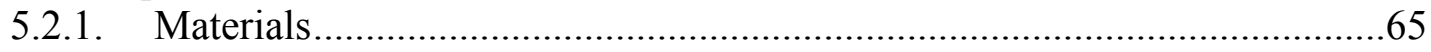

5.2.2. Construction of pseudo-ternary phase diagrams .........................................65

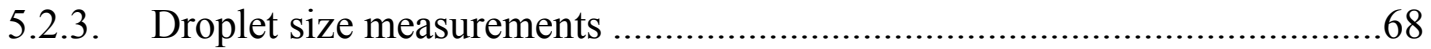

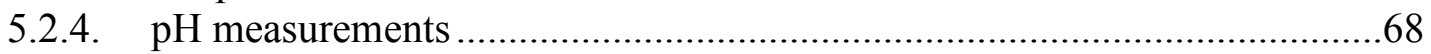

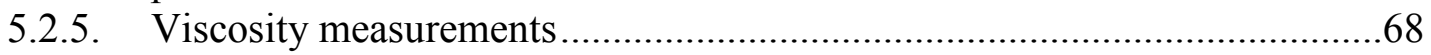

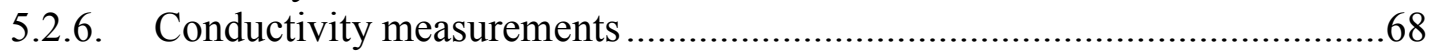

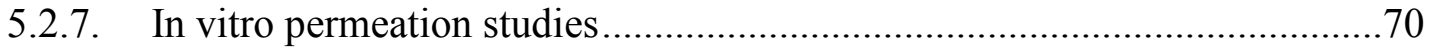

5.2.7.1. Skin preparation .......................................................................... 70

5.2.7.2. In vitro diffusion experiments ............................................................ 70

5.2.8. Accelerated microstructure stability testing ................................................71

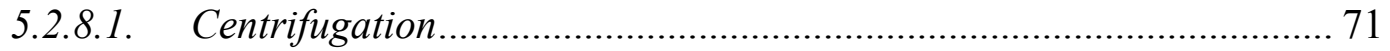

5.2.8.2. Thermal stability ........................................................................... 71

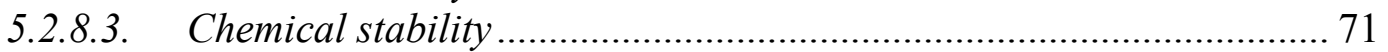

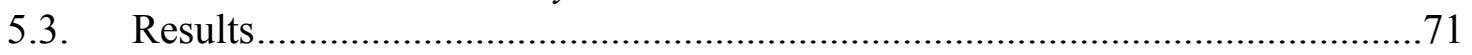

5.3.1. Construction of pseudo-ternary phase diagrams ..........................................71

5.3.2. Droplet size and $\mathrm{pH}$ measurements ........................................................72

5.3.3. Viscosity measurements.........................................................................72

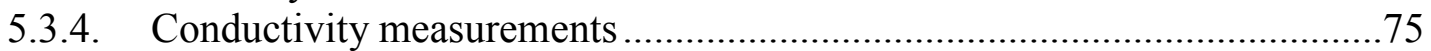

5.3.5. In vitro microemulsion skin permeation studies .........................................75

5.3.6. Accelerated microstructure stability testing ...............................................78

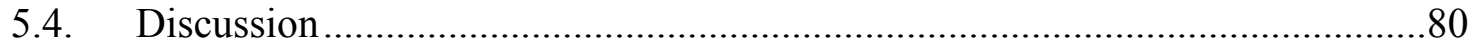

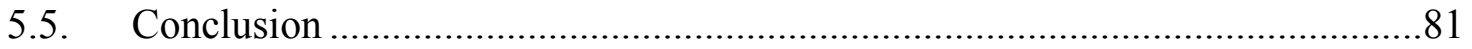

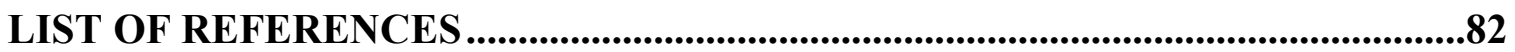

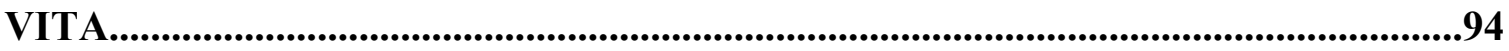




\section{LIST OF TABLES}

Table 2-1. Experiment formulations and the levels of variables according to an orthogonal experimental design L9 $\left(3^{3}\right)$

Table 2-2. Composition expressed as (w/w \%) for orally disintegrating film and orally disintegrating tablet formulations.

Table 2-3. Responses (drug encapsulation efficiency and percentages of drug released) of microparticle formulations.

Table 2-4. Optimum regression equations which influenced the percentages of drug

released.

Table 2-5. Comparison between the predicted and the actual values for encapsulation efficiency and $\mathrm{f}_{2}$ similarity factor testing for the three selected variable levels.

Table 2-6. Properties of orally disintegrating films and orally disintegrating tablet formulations.

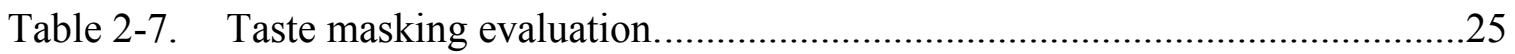

Table 3-1. The amounts of PVP adsorbed onto Sulfamerazine particles after milling at concentrations.

Table 4-1. Surface area and particle size analysis of various powder samples.

Table 4-2. Characteristics of mini-tablets. .56

Table 5-1. Formulation composition, $\mathrm{pH}$ and Z-average diameter at $25^{\circ} \mathrm{C}$.

Table 5-2. Cumulative permeated iodide $\left(\mathrm{Q}_{24}\right)$ and flux at steady-state (Jss) $\left(\mathrm{mg} / \mathrm{cm}^{2} / \mathrm{h}\right)$ of selected formulations. 


\section{LIST OF FIGURES}

Figure 2-1. Response surface plot which relates drug encapsulation efficiency (\%) as a function...........................................................................................16

Figure 2-2. Predicted and observed release profiles of three validated formulations. ....20

Figure 2-3. Dissolution profiles of Chlorpheniramine Maleate.......................................23

Figure 3-1. Particle size and volume percentiles of particles in suspensions below 1 $\mu \mathrm{m}$.

Figure 3-2. Particle size and volume percentiles of particles below $1 \mu \mathrm{m}$ of lyophilized formulations. ...............................................................................

Figure 3-3. Dissolution profiles of Sufamerazine...........................................................

Figure 3-4. DSC patterns of various powder samples. .....................................................40

Figure 4-1. DSC patterns of various powder samples....................................................49

Figure 4-2. X-ray powder diffraction patterns of various Prednisone powder samples.

Figure 4-3. Kinetic solubility of Prednisone powder samples.......................................52

Figure 4-4. SEM micrographs of Prednisone powder samples. ........................................54

Figure 4-5. Dissolution profiles of Prednisone powder samples.......................................57

Figure 4-6. Dissolution profiles of core mini-tablets....................................................59

Figure 4-7. Dissolution profiles of coated mini-tablets....................................................60

Figure 4-8. Dissolution profiles of mini-tablets after 1-month stability test. ..................62

Figure 4-9. Photos of Formulation 1 and Formulation 4...............................................63

Figure 5-1. Pseudo-ternary phase diagrams of mixtures composed of oil (Capryol $90 \AA$ ), water, surfactant (Span 20), and cosurfactant (ethanol) at various

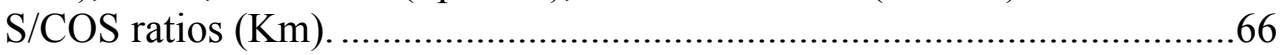

Figure 5-2. Formulation selection from pseudo-ternary phase........................................67

Figure 5-3. A schematic demonstration for the water interface of microemulsions. ......73

Figure 5-4. The change of dynamic viscosity as a function of water.............................74 
Figure 5-5. Conductivity of microemulsion formulations along dilution line L20

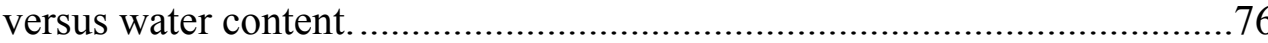

Figure 5-6. Permeation profiles of KI formulations. ................................................. 77

Figure 5-7. Test papers for sample stability test........................................................ 


\section{LIST OF ABBREVIATIONS}

$\begin{array}{ll}\text { ANN } & \text { Artificial neural network } \\ \text { ANOVA } & \text { Analysis of variance } \\ \text { API } & \text { Active pharmaceutical ingredient } \\ \text { BCS } & \text { Biopharmaceutics classification system } \\ \text { COS } & \text { Co-surfactant } \\ \text { CV } & \text { Coefficient of variation } \\ \text { DOE } & \text { Design of Experiment } \\ \text { DSC } & \text { Differential scanning calorimetry } \\ \text { EE } & \text { Encapsulation efficiency } \\ \mathrm{f}_{2} & \text { Similarity factor } \\ \text { GI } & \text { Gastrointestinal } \\ \text { HLB } & \text { Hydrophilic-lipophilic balance } \\ J_{\text {Ss }} & \text { Steady-state flux value } \\ \text { KI } & \text { Potassium Iodide } \\ \text { Km } & \text { Surfactant/cosurfactant ratio } \\ \text { ODF } & \text { Orally disintegrating film } \\ \text { ODT } & \text { Orally disintegrating tablet } \\ \text { PEG } & \text { Poly (ethylene glycol) } \\ \text { PVP } & \text { Polyvinylpyrrolidone } \\ \text { R } & \text { Multiple correlation coefficients } \\ \text { RH } & \text { Relative humility } \\ \text { S } & \text { Surfactant } \\ \text { SEDDS } & \text { Self-emulsification drug delivery system } \\ \text { SEM } & \text { Scanning electron microscope } \\ \text { SGF } & \text { Simulated gastric fluid } \\ \text { SS } & \text { Simulated saliva } \\ \text { T3 } & \text { Triiodothyronine } \\ \text { T4 } & \text { Thyroxine } \\ \text { UV } & \text { Ultraviolet } \\ \text { W/O } & \text { Water/Oil } \\ \text { W/V } & \text { Weight/Volume } \\ \text { W/W } & \text { Weight/Weight } \\ & \end{array}$




\section{CHAPTER 1. INTRODUCTION}

Due to the lack of pediatric formulations, the regulatory agencies have initiated continual emphasis on the necessity for developing novel pediatric formulations. In response, pharmaceutical companies have developed various novel pediatric formulations some of which have already been launched into the market.

To develop a satisfactory pediatric formulation, an appropriate route of drug administration is the prerequisite and can be selected from one of the followings: oral, topical/transdermal, buccal, rectal, nasal, pulmonary, ocular, injectable, etc. ${ }^{1}$

\subsection{Oral Pediatric Formulation}

In general, oral delivery of pediatric drugs is preferred over other delivery routes since it is convenient, economical, and user-friendly. Based on Strickley and co-workers'

classification, ${ }^{1}$ pediatric oral formulations were divided into two types: (1) Ready-to-Use formulations, which do not need manipulation prior to dosing; (2) Manipulation-Required formulations, which require manipulation under some instructions prior to dosing. In contrast to regular adult oral formulations such as tablets and capsules, pediatric oral formulations are likely to contain some specific features to achieve good patient compliance for pediatrics. In response, several customized oral pediatric formulations were by far developed.

\subsubsection{Orally disintegrating tablets (ODTs)}

ODTs are designed to disintegrate in the oral cavity instead of the GI tract and therefore a rapid disintegration, usually in less than a minute, is necessitated under the exposure of saliva. Previous studies indicated that there has been a remarkable growth of ODT demand since the majority of patients preferred (more than $85 \%$ of patients) and would purchase ODTs ( $70 \%$ of patients) rather than other regular tablet formulations. ${ }^{2}$ ODTs can be produced by different techniques, which include freeze-drying process, molding/cotton-candy process, and compaction process. ${ }^{3}$ To scale-up ODT preparation, conventional tablet compression method and instrument with some modifications are applicable. ${ }^{4}$

Unlike regular tablets needed to be swallowed integrally, ODTs eliminate the necessity of tablet swallowing and thus fairly suitable for elder children. It is worth noting that several ODT products have already been launched to commercial market for pediatrics such as Prevacid ${ }^{\circledR}$ (lansoprasole), Zofran ${ }^{\circledR}$ (ondansetron), and Clarinex ${ }^{\circledR}$ RediTabs ${ }^{\circledR}$ (desloratadine). ${ }^{1}$ In view of challenges of ODT development, one obstacle attributes to the unpleasant taste of some APIs that is essentially associated to taste masking. Tablet film-coating is not applicable for ODTs since ODT is disintegrated in the oral cavity and consequently unpleasant taste of drug is sensed. Coating in the powder 
level is one approach to achieve taste masking for ODTs. Xu and co-workers developed Famotidine: Eudragit ${ }^{\circledR}$ EPO: colloidal silicon dioxide: polyethylene glycol 400 microspheres using spray-drying technique and then these microspheres are incorporated into ODTs. ${ }^{5,6}$ Conversely, Khan and co-workers prepared ondansetron $\mathrm{HCl}$ in Eudragit ${ }^{\circledR}$ EPO complex using precipitation method, followed by formulating ODTs. Moreover, if drug content is relatively low in ODTs (less than $20 \mathrm{mg}$ ), the addition of flavors and sweeteners is another feasible approach. ${ }^{1}$

\subsubsection{Film strips/Orally disintegrating films (ODFs)}

The first ODF product (Listerine PockerPaks ${ }^{\circledR}$ ) was introduced to the market by Pfizer in 2001 and over time this novel formulation has gained more interest on popularity. ODF is a film strip which disintegrates rapidly on the tongue without being chewed or being swallowed. Therefore, ODFs can be administered by pediatric patients at ease. Two techniques have been developed to prepare ODFs, including casting method and hot-melt extrusion method. Casting method involves dissolution and/or dispersion of active pharmaceutical ingredients (APIs) and various excipients in an aqueous medium, medium casting, and drying. Hot-melt extrusion method, as the relatively new method, includes extrusion of melted drug and/or polymers' complex, casting, cooling, and drying. In order to develop a satisfactory ODF, various excipients are desirable to obtain acceptable performance such as robustness, content uniformity, taste masking, rapid disintegration, appearance, etc. ${ }^{7}$ Film-forming polymer is the primary component of ODF and play a critical role in film's mechanical properties. A number of polymers were utilized individually or in a combination as the backbone of film strips and these polymers include modified starch (maltrodextrin) ${ }^{8,9}$ hypromellose, ${ }^{10}$ andpyrrolidone. ${ }^{11}$ In addition, a balance of plasticizer and surfactant in film's formula is pursued to exhibit good spreadability and elasticity properties. Taste masking should be reached if APIs in the ODF are unpleasant. The addition of taste masking agents is the most prevalent way to attenuate the undesirable taste. On the other hand, the method of coating in the powder level may be employed for the purpose of taste masking since microparticles could be incorporated in film matrix successfully. ${ }^{12}$

\subsubsection{Mini-tablets}

Apart from ODT and ODF, mini-tablet is another option to achieve formulation swallowability for pediatric patients. Mini-tablet is defined as a tablet with a diameter less than $3 \mathrm{~mm} .{ }^{13}$ As a replacement of regular tablets, mini-tablets can be swallowed by children less than 10 years old at their ease. ${ }^{14}$

There is a potential for mini-tablets to be applied widely as a pediatric formulation due to (1) its unique advantages such as regular shape, good appearance, acceptable mechanical properties, and uniform content; (2) the feasibility of mini-tablets $\left(1 \mathrm{~mm}\right.$ and $2 \mathrm{~mm}$ in diameter) manufacturing using regular tablet compression machine. ${ }^{15}$ 
In addition, mini-tablets were utilized in other drug route of administration instead of oral. For example, some researchers applied coated mini-tablet as an ophthalmic insert for drug control release in eyes. ${ }^{16}$

\subsubsection{Nanocrystals (constituted to a suspension)}

Powder (granule) is a type of "Manipulation-required formulation" and has been utilized very commonly as a pediatric formulation. Prior to the application, this formulation is necessary to be constituted to a suspension formulation. Apart from API powder, other excipients can also be added into the powder blend, including flavor agents, color agents, sweeterners, dispersing agents, buffering agents, preservatives, viscosity modifiers, etc. ${ }^{1}$

In contrast to regular powder in micron level, nanoparticle has an increase of dissolution rate and saturated solubility, especially for poorly soluble APIs, which may result in higher oral bioavailability. Moreover, nanoparticles are easier to adhere to GI membrane and consequently enhance oral bioavailability further. ${ }^{17}$

Currently there are several techniques for yielding nanosuspensions. In general, these methods can be divided into two main categories: "bottom-up" technology and "top-down" technology. The "bottom-up" technology basically is those methods via recrystallizing nanocrystals from solution. Due to inherited limitations of "bottom-up" technology such as wide variation in particle size distribution, solid state transformation (unstability), and scale-up difficulty, this technology is not widely applied. ${ }^{18}$ On the other hand, the "top-down" technology such as wet bead milling and high pressure homogenization which involves methods about reducing particle size from micron range is more popular. Wet bead milling involves the milling process of powders in aqueous medium by colliding with glass/pearl beads. Conversely, high pressure homogenization is a relatively new technology and nanoparticles are achieved using more sophisticated equipments. One example is the microfluidizer, where particles were milled by two highvelocity jet streams. The other most-common equipment is piston-gap homogenisers. In this equipment, a drug solution is pushed through a gap by the aid of large force applied by a piston, followed by the boiling of liquid droplets. Sequentially these gas bubbles fracture with the formation of nanocrystals. ${ }^{19}$

\subsection{Transdermal Formulations}

When oral or parental route of administration is inapplicable or encountered with insufficient patient compliance, alternative routes such as transdermal delivery is pursued with good pediatric patient compliance.

Transdermal delivery has been utilized for several decades and considered as a non-invasive route of administration. Although, transdermal delivery is utilized in several

clinical situations, the main challenge for this delivery route has always been the drug 
dose and the skin permeability since skin function as a barrier to prevent external invasions. Nanotechnology has been utilized in transdermal drug delivery to improve permeation; and microemulsion is considered the second most popular colloidal system which has been used for dermal applications. ${ }^{20}$

Microemulsion is a multicomponent system composed of water, oil, surfactant, and cosurfactant. ${ }^{21}$ Here, we mention several advantages of microemulsions: First, the procedures for preparing microemulsions are simple, inexpensive, and rapid. Second, microemulsions have shown to enhance the permeability of hydrophobic and hydrophilic compounds, ${ }^{22}$ either by decreasing the skin resistance or by increasing the concentration gradient of the drug diffusion. ${ }^{23}$ In addition, the nano-size droplets of microemulsion are more likely to adhere to the skin surface and penetrate into the skin. ${ }^{22}$ In addition, microemulsions as compared to regular emulsions (microns size), are thermodynamically stable during storage, packaging, and transportation due to the lack of fixed curvature and the low interfacial tension. 


\section{CHAPTER 2. EVALUATION OF CHLORPHENIRAMINE MALEATE MICROPARTICLES IN ORALLY DISINTEGRATING FILM AND ORALLY DISINTEGRATING TABLET FOR PEDIATRICS *}

\subsection{Introduction}

In recent years, pediatric formulations have gained more attention from private and governmental institutes. Although there were many governmental incentives offered to pharmaceutical companies to design and improve new pediatric formulations, developing a satisfactory pediatric formulation is still a challenging task for many reasons: (1) Overwhelming majority of active pharmaceutical compounds are poorly soluble and/or poorly absorbable which cause low bioavailability; (2) Some pediatric formulations such as powders or granules have tremendous dose strength fluctuations while dosing; (3) Swallowing appropriateness may not be achieved easily for children less than 10 years of age; (4) Many active pharmaceutical compounds or excipients have unpleasant taste. ${ }^{1}$ Despite critical issues such as low solubility/stability and dose strength fluctuations, swallowing inappropriateness and unpleasant taste are the two most critical obstacles that cause very low patient compliance, specifically for the pediatric patients.

Since a large number of active pharmaceutical ingredients have unpleasant taste, various methods have been developed by formulation scientists to attenuate the undesirable taste. It has been reported that appropriate flavors, sweeteners, and amino acids were simply incorporated into the formulations for the purpose of taste masking. ${ }^{24}$ Lipophilic vehicles were typically used to prevent the contact between bitter taste drug molecules with taste buds on the tongue by way of inhibiting the sense of bitterness. ${ }^{24}$ Complexing agents such as cyclodextrin were used to bind bitter taste drug molecules inside its hydrophobic cavity to mask bitterness. ${ }^{25,26,27}$ Taste masking was also achieved via binding of drug molecules into ion-exchange resins. ${ }^{24,28}$ Apart from all these above mentioned taste-masking techniques, the utilization of a $\mathrm{pH}$-dependent polymer as a coating material to avoid the contact between taste buds on the tongue with bitter tasting drugs is a feasible and economic approach. Selectively, Eudragit ${ }^{\circledR}$ EPO, a pH-dependent copolymer, is insoluble in saliva ( $\mathrm{pH}$ 6.8) but dissolves rapidly in gastric fluid ( $\mathrm{pH} \mathrm{1.5-2).}$ Therefore, film coating with Eudragit ${ }^{\circledR}$ EPO can inhibit the release of drug from a tablet in the saliva and mask the bitter taste. ${ }^{29}$ This polymer was also used as a moisture protectant during tablet coating. ${ }^{29}$ Additionally, Eudragit ${ }^{\circledR}$ EPO is suitable for powder coating. In contrast to other techniques of making microparticles, spray drying technique has a continuous, automated and quick process for producing products and therefore can be scaled up for industrial uses. In the past, API-Eudragit ${ }^{\circledR}$ EPO has been utilized to synthesize microparticles for masking the unpleasant taste of drug powders and loaded

* : Adapted with permission. Lou H, Liu M, Qu W, Hu Z, Brunson E, Johnson J, Almoazen H. Evaluation of chlorpheniramine maleate microparticles in orally disintegrating film and orally disintegrating tablet for pediatrics. Drug Dev Ind Pharm. In Press. 
into oral dosage forms. ${ }^{5,6}$ In this study, we selected orally disintegrating tablet (ODT) because ODT are different from traditional tablets since they are designed to disintegrate in the oral cavity instead of the GI tract. Therefore, pediatric patients can avoid swallowing a whole tablet and consequently enhance patient compliance. However, some pre-school children still have difficulty grasping skills of administering ODT because they cannot follow instructions such as "no swallowing" or "no chewing" properly. This makes orally disintegrating film (ODF) a viable option. ODF is a film strip which disintegrates rapidly on the tongue without being chewed or being swallowed. In contrast to ODT, ODF can be administered to pediatric patients with more ease since it is likely that users are already familiar with the use of this category of products by using breathfreshening strips. ${ }^{7}$ It is noted that the manufacturing process of ODT is much simpler and more cost-efficient as compared to that of ODF. To prepare ODF, either solvent casting method with solvent involvement or hot-melt extrusion method with external heat input is needed. ${ }^{7}$ In contrast, ODT can be directly compressed from powder blends without solvent or heat. Here we claim that both of these formulations are suitable for pediatric populations with some inherited advantages and disadvantages.

In this study, Chlorpheniramine Maleate was chosen as the model drug since it has been widely used as an antihistamine drug for pediatric patients. This drug is highly water soluble and has acceptable bioavailability. ${ }^{30}$ However, it is still difficult to develop satisfactory pediatric formulations such as ODT and ODF without masking the bitter taste of Chlorpheniramine Maleate.

The objective of this study was to develop satisfactory ODT and ODF formulations that could mask the taste of Chlorpheniramine Maleate. For this purpose, API-Eudragit ${ }^{\circledR}$ EPO microparticles were synthesized using the spray drying technique. The formulation of microparticles was optimized depending on two criteria (relatively high encapsulation efficiency and low drug release rate). Furthermore, although the feasibility of compressing microparticles with other excipients into ODT has been reported by others, ${ }^{5,6}$ incorporating microparticles into ODF has not been investigated. In this study, we evaluated the feasibility of microparticle incorporation into both ODT and ODF formulations.

\subsection{Experimental Procedures}

\subsubsection{Materials}

Polyvinyl alcohol and Tween 20 were purchased from Sigma Aldrich (MO, USA). Chlorpheniramine Maleate was purchased from Spectrum chemical (NJ, USA). Glycerin was purchased from Fisher Scientific (MA, USA). Eudragit® EPO was kindly donated by Evonik (NJ, USA). Spray-dried Mannitol was kindly donated by SPI Polyols Pharma (DE, USA). Lycoat ${ }^{\circledR}$ RS720 was kindly donated by Roquette (IL, USA). Croscarmellose sodium was kindly donated by FMC Biopolymer (PA, USA). Magnesium Stearate was kindly donated by Mallinckrodt (MO, USA). 


\subsubsection{Preparation of microparticles}

API-Eudragit ${ }^{\circledR}$ EPO microparticles were prepared using the spray drying procedure conducted by Mohanan et al. with minor modification. ${ }^{31}$ Briefly, $250 \mathrm{mg}$ of Chlorpheniramine Maleate were weighed and dissolved in $5 \mathrm{~mL}$ of distilled water (aqueous phase). Preset amount of Eudragit ${ }^{\circledR}$ EPO was weighed and dissolved in preset volume of dichloromethane (organic phase). The above two phases were mixed by manual shaking and emulsified under ultrasonication for 1 minute (QSonica LLC, CT, USA). Sequentially the obtained w/o emulsion was immediately spray dried with a mini spray dryer B-290 (Buchi, Switzerland). Spray drying parameters were set accordingly: aspirator $100 \%$, pump speed $25 \%$, inlet temperature $50{ }^{\circ} \mathrm{C}$, and pre-set compressed gas flow (indicated by the rotameter Q-flow). Q-flow was applied to disperse solution into fine liquid droplets at the tip of two-fluid nozzle. Values of the weight ratio of Eudragit $\mathbb{}$ EPO to drug, the volume ratio of dichloromethane to water, and gas flow (Q-flow) were selected as depicted in Table 2-1. At the end of spray drying, microparticles were collected, rinsed with 1\% PVA solution, and then dried at room temperature under vacuum for 24 hours. All harvested dry microparticles were kept at $4{ }^{\circ} \mathrm{C}$ in isolated vials for further use.

\subsubsection{Design of experiments}

Physicochemical properties of microparticles are influenced by formula composition and by process variables. To optimize the microparticle formulations properties, an orthogonal experimental mathematical design $\mathrm{L}_{9}\left(3^{3}\right)$ was conducted as shown in Table 2-1.

The weight ratio of Eudragit $®$ EPO to Chlorpheniramine Maleate was defined by the independent variable $\left(\mathrm{X}_{1}\right)$, the volume ratio of dichloromethane to water was defined by the independent variable $\left(\mathrm{X}_{2}\right)$, and Q-flow (in millimeter) was defined as the independent variable $\left(\mathrm{X}_{3}\right)$. Three levels were selected for each independent variable. Each experiment for the same level was duplicated. Drug encapsulation efficiency and percentage of drug released at $1 \mathrm{~h}, 2 \mathrm{~h}, 3 \mathrm{~h}, 4 \mathrm{~h}, 8 \mathrm{~h}, 12 \mathrm{~h}$, and $24 \mathrm{~h}$ were selected as the key responses. Polynomial equations including linear model Equation 2-1 and quadratic model Equation 2-2 were utilized to fit the observed results using SAS software (SAS institute INC, NC, USA). The best-fit model was selected based on the multiple

correlation coefficient $\left(\mathrm{R}^{2}\right)$, coefficient of variation $(\mathrm{CV})$, adjusted multiple correlation coefficient (adjusted $\mathrm{R}^{2}$ ), and multi co-linearity of predictor variables. Bias was checked by comparing predicted and observed responses.

$$
\begin{gathered}
\mathrm{Y}=\mathrm{k}+\mathrm{a} \mathrm{X}_{1}+\mathrm{bX}_{2}+\mathrm{c} \mathrm{X}_{3} \\
\mathrm{Y}=\mathrm{k}+\mathrm{aX} \mathrm{X}_{1}+\mathrm{bX}_{2}+\mathrm{c} \mathrm{X}_{3}+\mathrm{dX}_{1} \mathrm{X}_{2}+\mathrm{eX}_{1} \mathrm{X}_{3}+\mathrm{fX}_{2} \mathrm{X}_{3}+\mathrm{gX}_{1}{ }^{2}+\mathrm{hX}_{2}{ }^{2}+\mathrm{iX}_{3}^{2}
\end{gathered}
$$


Table 2-1. Experiment formulations and the levels of variables according to an orthogonal experimental design $L 9\left(3^{3}\right)$.

\begin{tabular}{cccc}
\hline & \multicolumn{3}{c}{ Variables/Levels } \\
\cline { 2 - 4 } Formulation & $\mathbf{X}_{\mathbf{1}}$ & $\mathbf{X}_{\mathbf{2}}$ & $\mathbf{X}_{\mathbf{3}}$ \\
\hline 1 & 5 & 3 & 30 \\
2 & 5 & 5 & 37.5 \\
3 & 5 & 7 & 45 \\
4 & 7.5 & 3 & 37.5 \\
5 & 7.5 & 5 & 45 \\
6 & 7.5 & 7 & 30 \\
7 & 10 & 3 & 45 \\
8 & 10 & 5 & 30 \\
9 & 10 & 7 & 37.5 \\
\hline
\end{tabular}

$\mathrm{X}_{1}$ : The weight ratio of Eudragit ${ }^{\circledR}$ EPO to drug while keeping drug amount constant; $\mathrm{X}_{2}$ : The volume ratio of dichloromethane to water while keeping the volume of water constant;

$\mathrm{X}_{3}$ : Q-flow (mm). 


\subsubsection{Drug encapsulation efficiency}

Dry microparticles $(12.5 \mathrm{mg})$ were accurately weighed and dissolved in $25 \mathrm{~mL}$ of $80 \%$ acetonitrile with aid of sonication. The solution was filtered through a $0.22 \mu \mathrm{m}$ micro-porous membrane filter. Drug concentration was determined at $262 \mathrm{~nm}$ using a UV spectrometer (PerkinElmer, MA, USA). This experiment was repeated in three replicates. The drug encapsulation efficiency (\%) was calculated according to Equation 2-3.

$$
\text { Drug encapsulation efficiency }(\%)=\frac{\text { Actual drug loading }}{\text { Theoretical drug loading }} \times 100
$$

\subsubsection{In vitro release testes}

Dry microparticles $(150 \mathrm{mg})$ were weighed and transferred into a dissolution basket wrapped with a $0.45 \mu \mathrm{m}$ micro-porous membrane filter. The modified basket loaded with sample was suspended in $50 \mathrm{~mL}$ of simulated saliva $(\mathrm{pH}=6.8)$ under constant mild mechanical shaking at $37^{\circ} \mathrm{C}$. One milliliter was withdrawn at predetermined time points ( $1 \mathrm{~h}, 2 \mathrm{~h}, 3 \mathrm{~h}, 4 \mathrm{~h}, 8 \mathrm{~h}, 12 \mathrm{~h}$, and $24 \mathrm{~h})$, and an equal volume of fresh simulated saliva was replaced after each sampling point. Each retrieved sample was filtered through a $0.22 \mu \mathrm{m}$ micro-porous membrane filter, and then was analyzed by UV spectrophotometer at $262 \mathrm{~nm}$ after appropriate dilution.

\subsubsection{Checkpoint analysis}

Three variable checkpoints at three $\left(\mathrm{X}_{1}, \mathrm{X}_{2}, \mathrm{X}_{3}\right)$ levels: $(7.5,5,37.5)$, $(8.75,6,41.25)$ and $(10,3,37.5)$ were selected to validate and verify the robustness of the simulation model. Three replicates were performed for each checkpoint. Predicted and observed drug encapsulation efficiency values were compared using the Student $t$ test. In addition, by comparing the predicted and observed drug release profiles, the dissolution profile similarity factor $\left(f_{2}\right)$ was calculated according to Equation 2-4 and the application condition of $\mathrm{f}_{2}$ was specified to this experiment. Predicted and observed release profiles were considered similar if $f_{2}$ value was $\geq 50$.

$$
\mathrm{f}_{2}=50 \times \log \left\{\left[1+(1 / \mathrm{n}) \sum_{t=1}^{n}\left(\mathrm{R}_{\mathrm{t}}-\mathrm{T}_{\mathrm{t}}\right)^{2}\right]^{-0.5} \times 100\right\}
$$

Where $n$ is the number of time points, $R_{t}$ is the predicted percentage of drug release at $t_{\text {th }}$ time point, $T_{t}$ is the mean observed percentage of drug release at $t_{\text {th }}$ time point.

\subsubsection{Preparation of ODF and ODT}

ODF were casted based on the compositions presented in Table 2-2. Briefly, Lycoat RS720® was dissolved in a solution containing pre-calculated amounts of water, glycerin and Tween 80 under manual stirring. Microparticles (or Chloropheniramine Maleate powders) were uniformly dispersed (or dissolved) into the solution. This sample 
Table 2-2. Composition expressed as (w/w \%) for orally disintegrating film and orally disintegrating tablet formulations.

\begin{tabular}{ccccc}
\hline Formulation & $\begin{array}{c}\text { Film } \\
\text { (Control) }\end{array}$ & $\begin{array}{c}\text { Film } \\
\text { (Microparticle) }\end{array}$ & $\begin{array}{c}\text { ODT } \\
\text { (Control) }\end{array}$ & $\begin{array}{c}\text { ODT } \\
\text { (Microparticle) }\end{array}$ \\
\hline $\begin{array}{c}\text { Drug only } \\
\text { Microparticles (drug }\end{array}$ & 0.75 & N/A & 1.55 & N/A \\
equivalent of & N/A) & $(0.75)$ & N/A & 21.9 \\
microparticle) & & & & $(1.55)$ \\
Lycoate RS 720 & 41.125 & 31.25 & N/A & N/A \\
Glycerin & 5 & 5 & N/A & N/A \\
Tween 80 & 3.125 & 3.125 & N/A & N/A \\
Water & 50 & 50 & N/A & N/A \\
Mannitol & N/A & N/A & 93.7 & 73.6 \\
Croscarmellose Na & N/A & N/A & 4 & 4 \\
Mg stearate & N/A & N/A & 0.75 & 0.5 \\
\hline
\end{tabular}


was then allowed to stand until air bubbles dissipated. Thereafter, this obtained viscous sample was casted on a plastic platform using a $6 \mathrm{~mm}$ wet film stainless steel bar applicator (BYK Inc, CT, USA). The film was dried in a tray oven and was cut into multiple pieces of $3 \times 3 \mathrm{~cm}\left(9 \mathrm{~cm}^{2}\right)$ with the aid of BYK chart paper. ODT were also prepared based on the compositions presented in Table 2-2. Drug contents as well as weight of an ODT were pre-calculated based on those of an ODF. All powder ingredients were blended and compressed into a biconvex tablet with a diameter of $5.56 \mathrm{~mm}$ using a Carver hydraulic press (Carver INC, IN, USA). The compression force was kept constant at $1.5 \mathrm{kN}$.

\subsubsection{Characterization of ODF}

\subsubsection{Weight variation and content uniformity}

Six pieces of ODF $(3 \times 3 \mathrm{~cm})$ were accurately weighed to evaluate weight variations. For content uniformity evaluation, each film (six replicates) were dissolved in $25 \mathrm{~mL}$ of $0.1 \mathrm{~N} \mathrm{HCl}$ solution individually. Consequently, the resulting solutions were filtered through a $0.22 \mu \mathrm{m}$ microporous membrane filter. The drug concentration was determined by UV absorbance at $262 \mathrm{~nm}$.

\subsubsection{Tensile strength}

Tensile strength (tensile stress at break) of ODF was evaluated using dynamic mechanical analyzer equipped with a film clamp (TA instrument, DE, USA). A film was clipped into the clamp with one side fixed and the other side movable. The dimensions of the film were fed into the analyzer. The preloaded force was kept constant at $0.01 \mathrm{~N}$ then followed by ramping at $0.5 \mathrm{~N} / \mathrm{min}$ until the film broke. The temperature was maintained at $25{ }^{\circ} \mathrm{C}$ during all the experiments and the average tensile strength was calculated based on six replicates.

\subsubsection{In vitro disintegration}

In vitro disintegration testing of ODF was performed using the Pharmacopeia disintegration apparatus for capsules and tablets (Hanson Research, CA, USA). Each film strip was cut into an appropriate size $(n=3)$ and then carefully placed at the bottom of a disintegration basket tube. The system was maintained at $37^{\circ} \mathrm{C}$ and simulated saliva was chosen as the medium. Disintegration time was monitored until the film completely disintegrated into fine particles. 


\subsubsection{Characterization of ODT}

\subsubsection{Weight variation and content uniformity}

Six tablets were weighed to determine average weight variation and content uniformity, following the same procedures specified in Section 2.2.8.1 $(\mathrm{n}=6)$.

\subsubsection{Tensile strength}

Tablet hardness was measured using a manual tablet testing instrument (Vortex sales group, NC, USA). Tensile strength was calculated using Equation 2-5:

$$
\text { Tensile strength }=\frac{2 * \mathrm{~F}_{\mathrm{d}}}{\pi * \mathrm{D} * \mathrm{H}}
$$

Where $\mathrm{F}_{\mathrm{d}}$ is tablet hardness, $\mathrm{D}$ is tablet diameter, and $\mathrm{H}$ is tablet central cylinder thickness.

\subsubsection{Friability}

Friability was performed using a friabilator (Sotax, MA, USA). Twenty tablets of each formulation were first de-dusted, weighed and mixed with $3 \mathrm{~mm}$ glass beads to make a total weight of around $6 \mathrm{~g}$. Tablets and $3 \mathrm{~mm}$ glass beads were placed into the drum. After 100 revolutions, the tablets were first examined for appearance, and then weighed. Percent friability was calculated using Equation 2-6.

$$
\text { Percent Friability }=\frac{\text { initial weight }- \text { final weight }}{\text { initial weight }} \times 100 \%
$$

\subsubsection{In vitro disintegration}

The procedure for in-vitro disintegration testing was similar to the one specified in Section 2.2.8.3. One tablet was cautiously placed in the bottom of a disintegration basket tube. The average disintegration time was calculated from three replicates.

\subsubsection{Drug dissolution from ODF and ODT}

Dissolution tests were conducted at two different dissolution setups. Initially, to evaluate drug dissolution in the oral cavity, the USP basket apparatus (Distek INC, NJ, USA) was utilized. Three hundred milliliters of simulated saliva $(\mathrm{pH}=6.8)$ were maintained at $37^{\circ} \mathrm{C}$. One film or tablet was placed inside the basket and immersed in the dissolution medium. The rotation speed of the basket was kept at $50 \mathrm{rpm}$. The concentration of Chlorpheniramine Maleate was monitored using an in-line ultraviolet detector connected with fiber optic cable at $262 \mathrm{~nm}$ (pION, MA, USA).

Thereafter, to evaluate drug dissolution in the stomach, the USP paddle apparatus (Distek INC, NJ, USA) was utilized. Nine hundred milliliters of simulated gastric fluid 
$(\mathrm{pH}=1.2)$ were maintained at $37^{\circ} \mathrm{C}$. One film was hanged with a clip or one tablet was directly loaded into the vessel. The paddle rotation speed was kept at $50 \mathrm{rpm}$. Drug concentration was analyzed at $262 \mathrm{~nm}$ using the same detector.

\subsubsection{Taste masking evaluation}

Taste masking evaluation was performed using a single blind study according to a published design with minor modification. ${ }^{5}$ The bitterness of two ODFs and two ODTs were evaluated: ODF (control drug alone), ODF (coated microparticle), ODT (control drug alone), and ODT (coated microparticle). Ten adult volunteers were involved in this study. Each individual was asked to place each formulation on his/her tongue for 45 seconds and give a score based on his/her feelings about intensity of the bitterness. Intensity of bitterness was ranked from four numerals 0 to 3 , where $0=$ no bitterness, $1=$ slight bitterness, $2=$ moderate bitterness, and $3=$ strong bitterness. The average score of each formulation should be equal to or less than 1 to be considered acceptable for pediatric applications.

\subsection{Results}

\subsubsection{Drug encapsulation efficiency}

Drug encapsulation efficiency is defined as the ratio of the actual amount of encapsulated drug to the theoretical amount that was added. This indicator is a vital criterion which is used to evaluate the quality of microparticles. It has been illustrated that drug encapsulation efficiency could be significantly influenced by many formulation and process variables. For example, O'Donnell and McGinity concluded that the selection of the organic phase and the volume fraction of the organic and aqueous phases affected the encapsulation efficiency and the microparticle porosity. ${ }^{32}$ They also pointed out that the properties and amount of polymers were also influential on the drug encapsulation efficiency. ${ }^{32}$ Additionally, the process variables during the spray drying cycle are considered significant factors. In this study, gas flow (Q-flow) at the nozzle tip of the spray dryer was selected for further investigation because it controls the dispersion process of the spray dried solution into fine liquid droplets, thereby possibly affecting the drying process and drug encapsulation efficiency.

Values of drug encapsulation efficiency for all experimental batches were listed in Table 2-3. All experimental batches had acceptable encapsulation efficiency values which ranged from $60 \%$ to $90 \%$. After fitting data to both linear and quadratic models, the best-fit polynomial equation was determined as a modified quadratic model as shown in Equation 2-7, where the R-square value was 0.98. Analysis of variance (ANOVA) indicated that drug encapsulation efficiency was significantly influenced by experimental variables. Moreover, all predictor variables introduced in this equation were significant variables. 
Table 2-3. Responses (drug encapsulation efficiency and percentages of drug released) of microparticle formulations.

\begin{tabular}{ccccccccc}
\hline Formulation & \% EE & Y1h & Y2h & Y3h & Y4h & Y8h & Y12h & Y24h \\
\hline 1 replica a & 70.5 & 19.6 & 29.7 & 35.8 & 39.0 & 55.2 & 69.2 & 93.1 \\
1 replica b & 70.0 & 20.7 & 28.5 & 34.2 & 40.2 & 59.1 & 72.3 & 89.6 \\
2 replica a & 74.3 & 19.6 & 46.2 & 56.7 & 60.5 & 80.7 & 89.4 & 91.0 \\
2 replica b & 71.6 & 16.7 & 38.3 & 45.7 & 51.9 & 73.2 & 87.9 & 90.1 \\
3 replica a & 62.8 & 20.8 & 32.7 & 41.4 & 53.2 & 64.9 & 75.2 & 87.5 \\
3 replica b & 59.8 & 26.3 & 37.8 & 43.2 & 53.5 & 62.3 & 71.6 & 84.6 \\
4 replica a & 86.8 & 17.5 & 23.3 & 25.4 & 27.4 & 35.8 & 42.6 & 62.3 \\
4 replica b & 87.9 & 16.6 & 21.1 & 24.9 & 27.8 & 37.9 & 46.3 & 67.6 \\
5 replica a & 81.0 & 12.7 & 20.3 & 23.7 & 32.4 & 42.1 & 49.7 & 71.2 \\
5 replica b & 81.6 & 10.9 & 18.1 & 24.6 & 27.5 & 40.3 & 50.9 & 76.6 \\
6 replica a & 73.2 & 15.7 & 25.6 & 27.8 & 29.8 & 42.0 & 52.3 & 70.1 \\
6 replica b & 70.0 & 13.9 & 23.6 & 28.9 & 33.9 & 45.6 & 56.8 & 73.5 \\
7 replica a & 84.2 & 7.0 & 12.3 & 14.4 & 19.3 & 24.8 & 30.2 & 41.7 \\
7 replica b & 84.8 & 11.0 & 13.3 & 14.8 & 17.6 & 20.0 & 28.9 & 40.5 \\
8 replica a & 71.1 & 13.9 & 18.6 & 22.7 & 25.4 & 35.5 & 42.7 & 56.0 \\
8 replica b & 69.0 & 14.0 & 20.5 & 24.2 & 26.9 & 34.3 & 40.8 & 59.9 \\
9 replica a & 69.6 & 13.8 & 25.0 & 31.0 & 33.6 & 41.5 & 47.3 & 64.4 \\
9 replica b & 69.8 & 12.0 & 23.0 & 28.4 & 32.7 & 41.3 & 48.8 & 64.1 \\
\hline
\end{tabular}


Drug encapsulation efficiency $(\%)=-74.50+13.81 \mathrm{X}_{1}+6.42 \mathrm{X}_{2}+4.41 \mathrm{X}_{3}$ $+0.98 \mathrm{X}_{1} \mathrm{X}_{2}+0.18 \mathrm{X}_{1} \mathrm{X}_{3}-0.48 \mathrm{X}_{2} \mathrm{X}_{3}-0.81 \mathrm{X}_{1}^{2}$

(Eq. 2-7)

The influence of the three variables on drug encapsulation efficiency was schematically represented by three-dimensional surface response plots as seen in Figure 2-1. It was observed that drug encapsulation efficiency decreased as dichloromethane content increased. This observation may be related to the change in the porous nature of microparticle, which was explained by O'Donnell and McGinity. ${ }^{32}$ In addition, Q-flow was positively relevant to drug encapsulation efficiency. During the spray drying process, dichloromethane was evaporated in an extremely short period of time at a temperature above its boiling point. The hypothetical explanation is that the higher Q-flow dispersed the solution into finer droplets and then produced smaller microparticles that resulted in higher drug encapsulation efficiency. This result was also observed and explained by others where weakly bounded drug molecules on the surface of microparticles were more easily removed from larger but less dense particles during the preparation process. ${ }^{33}$ Finally, the drug encapsulation efficiency was also significantly influenced by polymer amount; however, as observed in Figure 2-1, this influence was parabolic and intertwined by interactions between polymer variable and other variables.

\subsubsection{In vitro release tests}

Percentage of drug release values were listed in Table 2-3. The slowest drug release percentage was around $40 \%$ at $24 \mathrm{~h}$ (Formulation 7 ) and the highest one was around $90 \%$ at $24 \mathrm{~h}$ (Formulations 1 and 2). Release profiles for all formulations resulted in a burst release in the first segment, followed by a zero-order release. This phenomenon was explained by Freiberg and Zhu who indicated that initial burst release is caused mainly by molecules extracted from microparticle shells, and the second zero-order release segment was primarily dependent on molecules diffusing through the matrix. ${ }^{34}$ Statistical analysis for the polynomial equations was conducted to determine the relationship between drug release and variables $\left(\mathrm{X}_{1}, \mathrm{X}_{2}, \mathrm{X}_{3}\right)$. Percentages of drug released at predetermined time points were fitted to both linear and quadratic mathematical equations. It was noted that although the quadratic model provided an equation with a better R-square value, multicollinearity issue may result in insignificance and inaccuracy of statistical analysis using this model. Therefore, the linear model was chosen for drug release simulation. Linear equations of each time point were described in Table 2-4. Analysis of Variance (ANOVA) of all regression equations indicated drug release was mainly determined by experimental variable levels. Multiple correlation coefficients $\left(\mathrm{R}^{2}\right)$ which were close to 1 indicated that the developed equations in Table 2-4 had a good fit to accurately describe the drug release data. The content and composition of polymers are critical for the release of drug from microparticles. ${ }^{35}$

In our experimental design, the weight ratio of polymer to drug ranged from 5 to 10 and was the most significant factor. The drug release rate decreased remarkably as polymer (Eudragit ${ }^{\circledR}$ EPO) content increased. Our explanation is that drug molecules are more difficult to penetrate through the microparticle shell which contains more amounts 

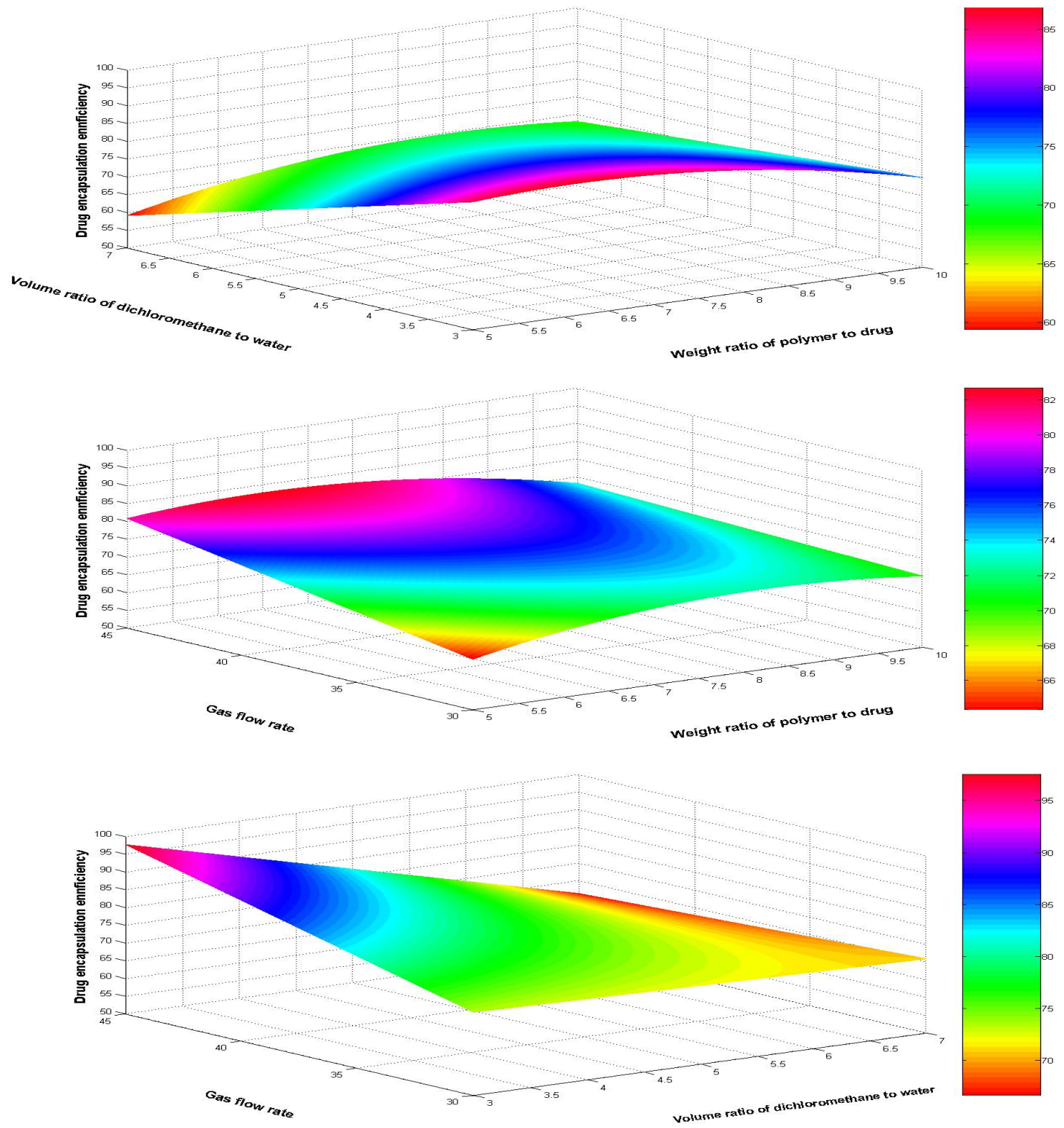

Figure 2-1. Response surface plot which relates drug encapsulation efficiency (\%) as a function.

(a) Top: Weight ratio of polymer to drug and volume ratio of dichloromethane to water (Q-flow at $37.5 \mathrm{~mm}$ );

(b) Middle: Weight ratio of polymer to drug and Q-flow (volume ratio of dichloromethane to water at 5);

(c) Bottom: volume ratio of dichloromethane to water and Q-flow (weight ratio of polymer to drug at 7.5). 
Table 2-4. Optimum regression equations which influenced the percentages of drug released.

\begin{tabular}{ccccccc}
\hline $\begin{array}{c}\text { Percentage of } \\
\text { Drug Release }\end{array}$ & $\begin{array}{c}\text { Intercept } \\
\text { Constant } \mathbf{( \% )}\end{array}$ & $\begin{array}{c}\text { Coefficient a } \\
\left(\mathbf{X}_{\mathbf{1}}\right)\end{array}$ & $\begin{array}{c}\text { Coefficient b } \\
\mathbf{( X}_{\mathbf{2}}\end{array}$ & $\begin{array}{c}\text { Coefficient c } \\
\left(\mathbf{X}_{\mathbf{3}}\right)\end{array}$ & P-value & R-square \\
\hline $\mathrm{Y}_{1 \mathrm{~h}}$ & 30.39 & -1.73 & 0.42 & -0.10 & 0.001 & 0.68 \\
$\mathrm{Y}_{2 \mathrm{~h}}$ & 47.33 & -3.35 & 1.65 & -0.13 & 0.0002 & 0.74 \\
$\mathrm{Y}_{3 \mathrm{~h}}$ & 54.93 & -4.05 & 2.13 & -0.13 & 0.0003 & 0.73 \\
$\mathrm{Y}_{4 \mathrm{~h}}$ & 53.76 & -4.76 & 2.73 & 0.09 & $<0.0001$ & 0.80 \\
$\mathrm{Y}_{8 \mathrm{~h}}$ & 89.68 & -6.60 & 2.70 & -0.19 & $<0.0001$ & 0.82 \\
$\mathrm{Y}_{12 \mathrm{~h}}$ & 110.92 & -7.56 & 2.60 & -0.31 & $<0.0001$ & 0.86 \\
$\mathrm{Y}_{24 \mathrm{~h}}$ & 130.06 & -6.98 & 2.06 & -0.45 & $<0.0001$ & 0.90 \\
\hline
\end{tabular}


of the polymer and thereby prolonged the release time. Apart from the weight ratio of polymer to drug, a faster drug release was achieved as the volume ratio of dichloromethane to water increased. This variable was also noticed to have an influence on drug release rate by other researchers. ${ }^{36}$ It is important to note that evaporating a larger volume of dichloromethane can possibly result in microparticle shell which is less dense and more porous, thereby results in faster drug release. Additionally, the third variable (Q-flow) was noticed to have insignificant influence on drug release.

As a result, microparticles coming from Formulation 7 were selected for loading into ODF and ODT for two primary reasons: (1) drug encapsulation efficiency was relatively high (around 84\%); (2) drug release rate was relatively slow (only around 10\% released within 1 hour and 40\% released within 24 hour).

\subsubsection{Checkpoint analysis}

Three checkpoints $\left(\mathrm{X}_{1}, \mathrm{X}_{2}, \mathrm{X}_{3}\right)$ at three variable levels $(10,3,37.5)(7.5,5,37.5)$, and $(8.75,6,41.25)$ were applied to validate the robustness of the developed models. Predicted and observed drug encapsulation efficiency values are listed in Table 2-5. The predicted drug encapsulation efficiency values of these validated points calculated from Equation 2-7 were not significantly different from the actual measured values $(\mathrm{P}>0.05$, statistical student t-tests). Predicted and observed drug release profiles were plotted in Figure 2-2. All similarity factor $\left(f_{2}\right)$ values in Table 2-5 were above 50, which suggest good similarity between predicted and observed release profiles. It is clear from these results that the values calculated for percentages of drug released according to the polynomial model can predict the observed drug release profiles. Moreover, these simulated models could also be utilized to predict drug encapsulation efficiency and drug release profile for other new untested formulations.

\subsubsection{Characterization of ODF and ODT}

The ODF and ODT formulations are listed in Table 2-2. The developed ODF contains Lycoat as a film forming polymer, glycerin as a plasticizer, Tween 80 as a surfactant, and drug (microparticles). Physicochemical and mechanical characterization values are listed in Table 2-6. The average weight of ODF control was $87.9 \pm 2.4 \mathrm{mg}$ and the average weight of ODF with microparticle was $86.3 \pm 4.0 \mathrm{mg}$. Thus, this produced films had similar weights whether drug was dissolved in or microparticles were dispersed in the casting solvent. Acceptable drug content uniformity was also achieved for both ODFs. The average drug content was approximately $1.3 \mathrm{mg}$, which is suitable strength for prescribed pediatric dose of 1 to $2 \mathrm{mg}$. The drug content and the weight of an ODT were aimed to be identical to those of an ODF. Thus, theoretical drug percentage in powder blends and in tablet weights were pre-matched. Consequently, the obtained data demonstrated that ODT controls and ODT with microparticle had extremely small weight variation and uniform drug content which closely matched their theoretical values. 
Table 2-5. Comparison between the predicted and the actual values for encapsulation efficiency and $f_{2}$ similarity factor testing for the three selected variable levels.

\begin{tabular}{cccc}
\hline Formulation $\left(\mathbf{X}_{\mathbf{1}} \mathbf{X}_{\mathbf{2}} \mathbf{X}_{\mathbf{3}}\right)$ & $\mathbf{( 1 0 ~ 3 ~ 3 7 . 5 )}$ & $\mathbf{( 7 . 5 ~ 5 ~ 3 7 . 5 )}$ & $\mathbf{( 8 . 7 5 ~ 6 ~ 4 1 . 2 5 )}$ \\
\hline Predicted EE\% & 75.1 & 77.0 & 72.4 \\
Observed EE\% & $74.3 \pm 1.5$ & $75.9 \pm 1.5$ & $73.8 \pm 2.2$ \\
P-value $(E E \%)$ & 0.48 & 0.31 & 0.40 \\
Similarity factor $\left(\mathrm{f}_{2}\right)$ & 83.8 & 72.4 & 76.5 \\
\hline
\end{tabular}




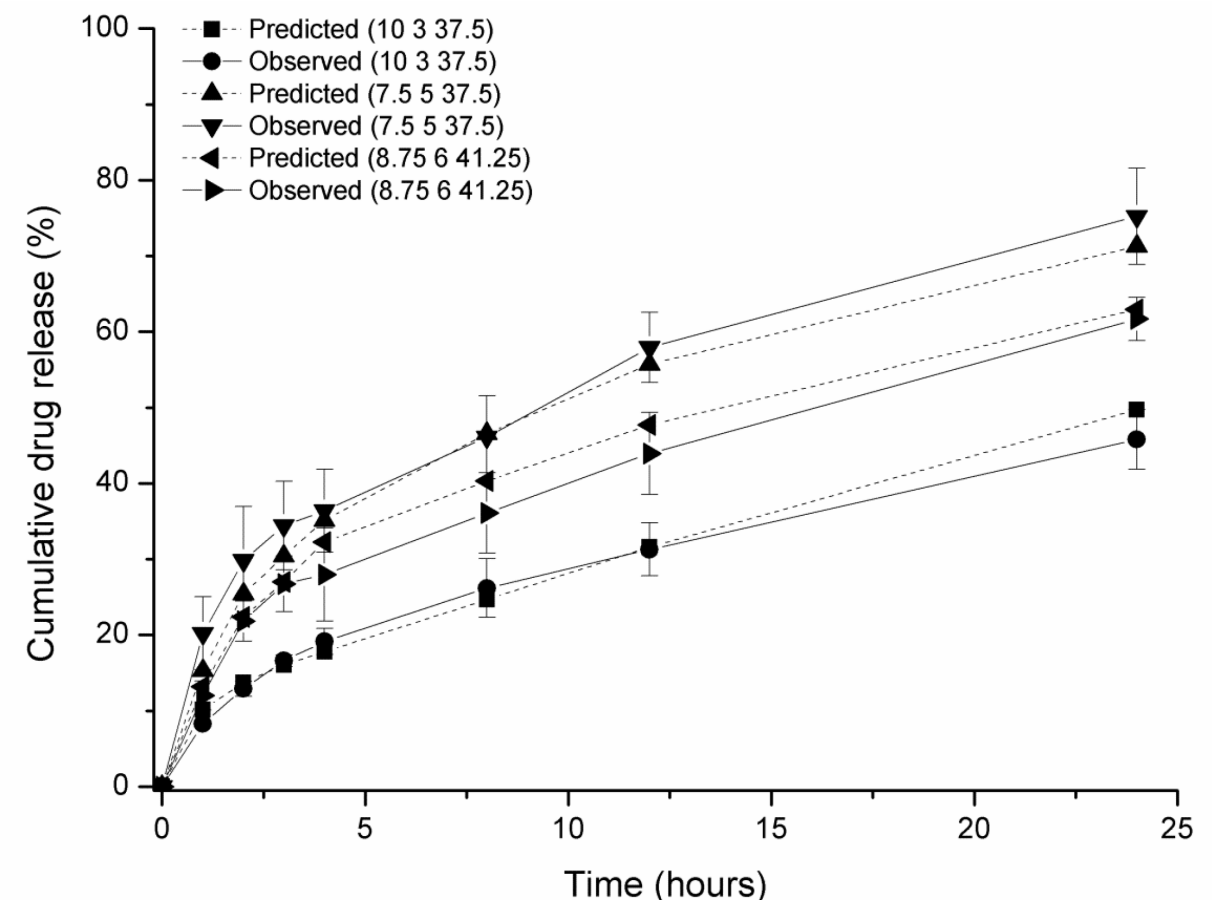

Figure 2-2. Predicted and observed release profiles of three validated formulations. 
Table 2-6. Properties of orally disintegrating films and orally disintegrating tablet formulations.

\begin{tabular}{ccccc}
\hline Formulation & $\begin{array}{c}\text { ODF } \\
\text { (Control) }\end{array}$ & $\begin{array}{c}\text { ODF } \\
\text { (Micropartile) }\end{array}$ & $\begin{array}{c}\text { ODT } \\
\text { (Control) }\end{array}$ & $\begin{array}{c}\text { ODT } \\
\text { (Microparticle) }\end{array}$ \\
\hline Weight (mg) & $87.9 \pm 2.4$ & $86.3 \pm 4.0$ & $86.2 \pm 2.2$ & $86.1 \pm 1.7$ \\
Drug content (mg) & $1.318 \pm 0.040$ & $1.307 \pm 0.052$ & $1.315 \pm 0.035$ & $1.332 \pm 0.0039$ \\
Disintegration (S) & $35.3 \pm 5.1$ & $36.0 \pm 3.6$ & $16.3 \pm 1.5$ & $23.7 \pm 1.5$ \\
Tensile strength (MPa) & $0.85 \pm 0.09$ & $0.51 \pm 0.09$ & $0.53 \pm 0.06$ & $1.00 \pm 0.11$ \\
Friability & & & $1.3 \%$ & $0 \%$ \\
\hline
\end{tabular}


Tensile strength is a vital mechanical property for evaluating the strength and robustness of ODF and ODT. ODF controls had significantly higher tensile strength than ODF with microparticles and therefore ODF controls were more robust. One possible explanation is that microparticles dispersed in the film strips may cause some strength. Tensile strength of films in this study was relatively lower than films made by other groups, ${ }^{8,9}$ both films kept good integrity during preparation, packaging, and storage conditions. On the other hand, ODT tensile strength is expressed as crushing strength. Tensile strength of ODT was noted to significantly increase with the addition of microparticles which may be due to higher elasticity of copolymer Eudragit ${ }^{\circledR}$ EPO. Disintegration testing demonstrated that both ODF and ODT disintegrated quickly. Disintegration time between the two ODF formulations was not significantly different $(\mathrm{P}>0.05)$. However, there was a significant difference between ODF and ODT formulations and between the two ODT formulations $(\mathrm{P}<0.05)$. Both ODFs disintegrated completely within 40 seconds, which is suitable for the orally disintegrating drug delivery systems. ODT had a relatively shorter disintegration time due to the inclusion of superdisintegrant croscarmellose sodium in the formula. The ODT controls disintegrated significantly faster than the ODT with microparticles. This result matched their tensile strength results. ODT with microparticles exhibited comparable results to the ODT which disintegrated within 10 to 30 seconds and had a tensile strength of $1 \mathrm{MPa} .{ }^{37}$ Friability results indicated that ODT with drug microparticles should have adequate robustness during package, storage, and transportation.

\subsubsection{Drug dissolution from ODF and ODT}

The taste of solid formulation is relevant to its drug content and its dissolution rate. In contrast to conventional taste masking techniques such as artificial flavors and sweeteners, a coating layer applied onto drug powders has been verified as a better approach to inhibit contact between drug molecules and taste bud sensors. ${ }^{5,6}$ It has been reported that a slow drug release for only few minutes at the initial stage could reduce unpleasant sensations associated with bitter taste of drugs. ${ }^{38}$ Thus, taste masking of Chloropheniramine Maleate can be achieved by reducing the initial drug dissolution rate. Dissolution profiles for controls and for microparticles in ODF and ODT at different media are presented in Figure 2-3. Both ODF and ODT loaded with microparticles, dissolved very fast (90\% of total drug amount within 2 minutes) in simulated gastric fluid $(\mathrm{pH}=1.2)$. Hence, drug release was not hindered by microparticles in simulated gastric fluid. In contrast, Eudragit ${ }^{\circledR}$ EPO microparticles in either ODF or ODT slowed drug release (approximate $40 \%$ at 5 minute) in simulated saliva $(\mathrm{pH}=6.8)$ significantly $(\mathrm{P}<0.05)$ as compared to ODF and ODT controls (more than $90 \%$ released at 5 minute). The reason is that, in simulated saliva, drug molecules in ODF and ODT loaded with microparticles needed to diffuse out from Eudragit ${ }^{\circledR}$ EPO microparticle shell. Additionally, in simulated saliva, there was no significant difference for drug release from microparticles in either ODF or ODT. The initial drug burst release from microparticles was observed in both formulations. The burst release is a manifestation of two reasons: (1) The drug is hydrophilic (water soluble); (2) Eudragit ${ }^{\circledR}$ EPO is swellable and water permeable at pH higher than 5 where the micro-channels that exist within microparticle shells enhance 


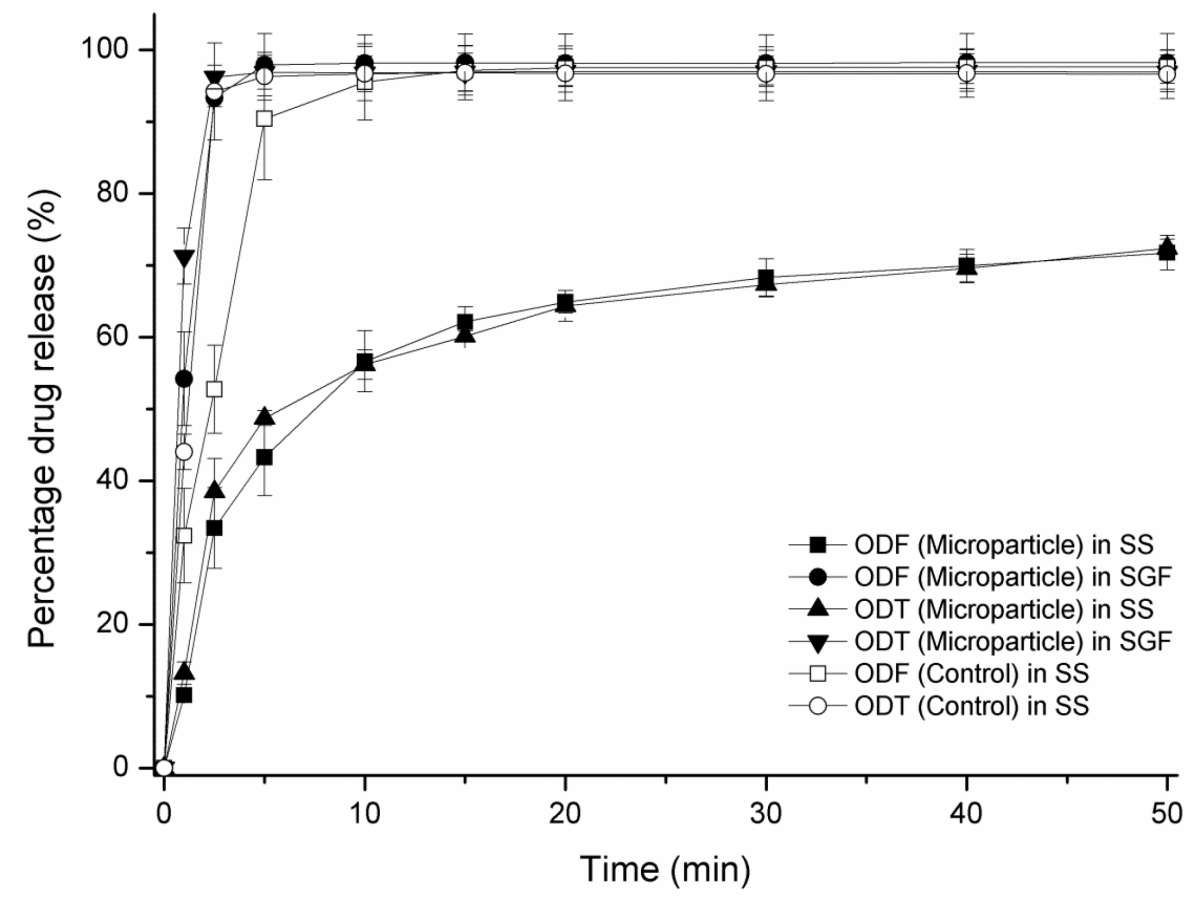

Figure 2-3. Dissolution profiles of Chlorpheniramine Maleate. 
drug diffusion. Drug dissolution in the simulated saliva was noticed to be more rapid under the experimental conditions listed in Section 2.2.11 as compared to those listed in Section 2.2.5. Obviously, the dissolution rate was influenced by experimental conditions. It is well understood that diffusion is the main mechanism of dissolution under the conditions listed in Section 2.2.5 while convection affected the dissolution listed in Section 2.2.11. In our study, we selected the conditions listed in Section 2.2.5 rather than those listed in Section 2.2.11 to evaluate drug release models of microparticles. It is mainly because relatively more experimental deviations among replicates (within same formulations) were observed in experiments conducted under the conditions listed in Section 2.2.11. Consequently, it became apparent that we would have some difficulty in building a precise model for establishing correlation between the variables and responses and obtaining optimized microparticle formulations.

\subsubsection{Taste masking evaluation}

Statistical scores of taste masking evaluations were listed in Table 2-7. In this experiment, the drug content in each unit dose was identical for all tested formulations (ODF and ODT). The results indicated that microparticles in either ODT or ODF successfully alleviated drug bitterness down to an acceptable level (score $\leq 1$ ). ODT with microparticles completely masked the bitterness of Chlorpheniramine Maleate(score $=0)$; in contrast, slight bitterness (score $=1.1$ ) was observed for ODT controls. In addition, ODF with microparticle assuaged the intensity of bitterness remarkably from moderate (score=2) to slight (score=1). We also noticed that ODT formulations had a better taste than ODF Formulations. Perhaps this could be explained by two reasons: (1) the excipient mannitol (filler) in ODT has a sweet taste, which could improve the taste of ODT; (2) ODF sticks easily on the tongue where released drug molecules and taste buds are in contact. This is contrary to ODT since microparticles are swallowed directly after disintegration. Therefore, we deduce that taste masking is associated with not only drug dissolution, but with other parameters such as the type of the solid dosage form and the selection of excipients.

\subsection{Discussion}

It is well known that classical solid oral dosage forms such as tablets or capsules are not appropriate for pediatric patients. Thus, alternative formulations are needed to enhance patient's compliance. Our intentions here were to enhance pediatric formulations by designing taste masked microparticles of Chlorpheniramine Maleate using Eudragit ${ }^{\circledR}$ EPO and incorporate these microparticles in pediatric friendly orally disintegrating film and orally disintegrating tablet. Microparticles have been considered a feasible approach

for taste masking. ${ }^{5,6,39}$ Unfortunately, this approach has required complicated preparation processes as compared to conventional methods for taste masking such as direct addition of flavor/sweetener. Despite the complexity of forming microparticles, microparticles have several advantages over flavor/sweetener additions particularly when the conventional methods are sometimes not effective. This is true when the bitterness 
Table 2-7. Taste masking evaluation.

\begin{tabular}{ccccc}
\hline $\begin{array}{c}\text { Score } \\
\text { (Bitterness) }\end{array}$ & \multicolumn{4}{c}{ Observation of Individual } \\
\cline { 2 - 5 } & $\begin{array}{c}\text { Film } \\
\text { (Control) }\end{array}$ & $\begin{array}{c}\text { Film } \\
\text { (Microparticle) }\end{array}$ & $\begin{array}{c}\text { ODT } \\
\text { (Control) }\end{array}$ & $\begin{array}{c}\text { ODT } \\
\text { (Microparticle) }\end{array}$ \\
\hline 0 (None) & 2 & 1 & 9 & 10 \\
1 (Slight) & 2 & 8 & 1 & \\
2 (Moderate) & 6 & 1 & & \\
3 (Strong) & 2 & 1 & 1.1 & 0 \\
Score Average & 2 & 1 & & \\
\hline
\end{tabular}


sensation overpowers the sweetness effect. ${ }^{24}$ Alternatively, microparticles provide practical solution because it inhibits the release of drug on the taste buds. In addition, it decreases the amount of excipients needed to mask the taste the bitterness and provide more convenient options for formulation scientists.

In this study, we approached the taste-masking project through optimizing experimental variables with the aid of Design of Experiments (DoE). DoE has been utilized widely as an organized approach to demonstrate the correlations between experimental variables and responses. ${ }^{40}$ During formulation developments, experimental variables can be either material variables (i.e. weight, volume, etc) or process variables (i.e. rate, force, etc), while experimental responses can be product quantitative attributes. DoE is highly utilized for small-batch formulation development and for scaling-up to large-batch processes. ${ }^{40}$ Since it is quite unreasonable to study all parameters (inputs) and all responses (outputs) simultaneously, DoE is generally utilized to optimize key inputs and identify key critical outputs. In this study, an orthogonal experimental design $\mathrm{L}_{9}\left(3^{3}\right)$ was introduced to investigate three important variables (the weight ratio of Eudragit ${ }^{\circledR}$ EPO to drug, the volume ratio of dichloromethane to water, and Q-flow). Orthogonal design, unlike empirical optimization methods, can emphasize the basic and eliminate the need for a large number of undesirable experiments. ${ }^{41}$ As part of the design, we correlated key variables and responses using polynomial regression mathematical analysis which has been verified as an effective tool to develop formulations. ${ }^{33,42,43,44}$ The results we obtained indicated that polynomial regression can precisely identify the relationships between variables and responses and sequentially predict the performance of formulations made by same variables at other levels (see Equation 2-7 and Table 2-2). This model enabled us to select microparticles from Formulation 7 for further exploration due to relatively high drug encapsulation efficiency (around 84\%) and slow drug release rate (only around $40 \%$ released within 24 hour). It is noticeable that the models we developed in this study could only be applied for experimental variables under a finite range $\left(X_{1}\right.$ from 5 to $10, X_{2}$ from 3 to $7, X_{3}$ from 30 to 45$)$. In addition, the surface response analysis cannot satisfy all formulation development requirements (e.g. a large number of variables included in formulation process) since polynomial regression might not be practical for variables with a high-order dimensionality. ${ }^{45}$ Thus, in these cases artificial neural networks (ANN) is considered as an alternative. ${ }^{45,46}$ We explored two oral dosage forms (ODF and ODT) which are suitable for pediatric patients. Since unpleasant taste of the drug has already been attenuated by the formation of microparticles, swallowing appropriateness is the other main concern for formulation selection. Unfortunately, pediatric patients have difficulty to swallowing regular solid dosage forms. And although liquid formulations are acceptable for children; its low portability and drug stability may become an issue. On the premise of easy administration and swallowing, ODF and ODT are concerned by pharmaceutical scientists. ${ }^{7}$

In this study, microparticles were successfully incorporated into both dosage forms and the release profile of microparticles did not change significantly as dosage forms (ODF or ODT) changed. In terms of acceptable taste-masking efficiency, these two dosage forms with microparticle loading could serve as the first front for the oral delivery of other bitter drugs for pediatric population. However, although these two dosage forms 
could both be successfully produced in small batches, there might be challenges for future scaling-up and large-batch production. Formulation scientists and process engineers might utilize Design of Experiment (DoE) as the key to overcome these challenges.

\subsection{Conclusion}

In this study, spray drying method is an appropriate method to produce microparticles with expected drug encapsulation efficiency and drug release profile. Drug encapsulation efficiency and release profile of microparticles made from various variable levels could be predicted accurately and the optimized microparticles were obtained with the aid of polynomial equations and response surface plots. The selected microparticles were formulated into ODF and ODT with reliable and reproducible preparation processes (small variations in weight as well as drug content, acceptable strength). The ODF and ODT disintegrated in short period of time, which are qualified for orally disintegrating dosage forms. Drug bitterness intensity was alleviated significantly in oral cavity, but Eudragit ${ }^{\circledR}$ EPO did not slow drug release in stomach. In conclusion, both ODF and ODT containing microparticles are potentially appropriate for pediatric uses. 


\section{CHAPTER 3. INFLUENCE OF SELECTED ANIONS ON MILLING OF SULFAMERAZINE NANOCRYSTAL *}

\subsection{Introduction}

Solid oral dosage forms are user-friendly and therefore preferred over many other dosage forms for systemic drug delivery. Typically, a satisfactory oral dosage form requires acceptable bioavailability which is related to drug solubility, dissolution, permeability and absorption. Unfortunately, many new drug molecules are hydrophobic and have low oral bioavailability. ${ }^{47}$ To overcome this challenge, formulators have made significant effort to enhance dissolution rate and solubility of drug molecules, particularly those classified in the biopharmaceutics classification system (BCS) as Class II (high permeability and low solubility) and Class IV (low permeability and low solubility). By far, several approaches to enhance dissolution rate and solubility have been developed. These include surfactants, ${ }^{48}$ amorphilization,${ }^{49}$ polymorph transformations, ${ }^{50}$ complexation, ${ }^{51}$ salt formation, ${ }^{52}$ and self-emulsification drug delivery system (SEDDS) ${ }^{53}$ Considering the current progress of formulation design concept from microsize level to nano-size level, nanocrystal technology has been matured and became an effective methodology for solubility and dissolution rate improvements. ${ }^{54,55,56,57,58,59,60} \mathrm{In}$ contrast to other dissolution rate improvement techniques, nanocrystallization has many benefits including no organic solvents involvement, high drug loading efficiency, and good reproducibility. ${ }^{54}$ Improvement of drug dissolution by nanocrystallization relies primarily on enlarging particles surface area and enhancing solubility of nanoparticles (Noyes-Whitney equation). Currently, there are several techniques for forming nanocrystals. In general, these methods can be divided into two main categories: "bottom-up" technology and "top-down" technology. The "bottom-up" technology includes recrystallizing nanocrystals from super-saturated solutions. Due to inherited limitations of "bottom-up" technology such as wide variation in particle size distribution, solid state transformation (physical instability), and scale-up difficulty, this technology is not widely applied. ${ }^{54}$ On the other hand, the "top-down" technology which involves methods for reducing particle size from micron range is more popular. One of the most important "top-down" methods is wet bead milling which is chosen for making nanocrystals quite frequently. ${ }^{61}$

In wet bead milling, drug particles and beads/pearls are milled in an aqueous medium and particle size is reduced by intensive collision. Due to higher surface energy, nanosuspensions have several folds of magnitude of internal energy as compared to that of conventional suspensions. For this reason, a large quantity of stabilizers is required to stabilize nanoparticles and either polymers or surfactants are used as stabilizers. During the last decade, extensive and significant research has been carried out to evaluate the

* : Adapted with permission. Lou H, Liu M, Qu W, Johnson J, Brunson E, Almoazen H. The influence of sodium salts (iodide, chloride and sulfate) on the formation efficiency of sulfamerazine nanocrystals. Pharm Dev Tech. In Press. 
influence of stabilizers on wet milling. It is recognized that this influence may be caused by the amount of stabilizer, ${ }^{62}$ its molecular weight, ${ }^{63}$ hydrophobicity/hydrophilicity, ${ }^{62}$ and functional groups. ${ }^{64}$ Moreover, other milling parameters such as type of drug, ${ }^{62,65}$ milling bead, ${ }^{65,66}$ milling frequency, ${ }^{67}$ and milling time ${ }^{67}$ were investigated.

As far as we know, wet milling has been explored in depth, but certain information on few aspects were not explored and are worthy of further investigation. For example, different salts (ions) could be dissolved in milling medium and their effects on milling efficiency is worth to be explored. The key question is: whether the addition of salts would positively or negatively affect milling? It must be noted that the influence of several anions on hydrocarbons' solubility (salting-in and salting-out effects) have been studied. ${ }^{68}$ According to Hofmeister ion series, the ranking of anions were based on the ability to increase surface tension of water and decrease the solubility of hydrocarbons in water. These anions were ranked as follows: $\mathrm{SO}_{4}{ }^{2-}>\mathrm{HPO}_{4}{ }^{2-}>\mathrm{OH}^{-}>\mathrm{F}^{-}>\mathrm{Cl}^{-}>\mathrm{Br}^{-}>\mathrm{NO}_{3}{ }^{-}>\mathrm{I}^{-}$ $>\mathrm{SCN}^{-}$. The anion to the left has the highest salting-out capability (increase surface tension and decrease hydrocarbon solubility in water) while anions to the right have the highest salting-in capabilities (decrease surface tension and increase hydrocarbon solubility in water). Although this phenomenon has been studied and documented, its implication on nanosuspension milling has not been studied yet. Briefly, the influence of salts is believed to "make" or "break" water structure. However, new theories derived from thermodynamic studies stated that the bulk water structure may not be enough linked to the effect of Hofmeister ions ranking. Interestingly, few scientists illustrated that ions, particularly anions, act directly on the first hydration shell of hydrocarbons and

the hydrocarbon's surface tension as well as the ion binding capability. ${ }^{68}$ The influence of anions in the Hofmeister series on hydrocarbon-water system has been applied in pharmaceutical development. For example, various salts were selected and utilized to prevent particle agglomeration during the coating process ${ }^{69}$ and to control the drug release pattern. ${ }^{70}$

The effect of salts as described above led us to hypothesize that wet bead milling might be influenced by the addition of salts since polymeric stabilizers play a critical role in maintaining drug nanoparticles physical stability. The objective in this study was to evaluate the influence of anions (sodium as cation) on nanocrystal formation by wet bead milling. Sulfamerazine was chosen as the model drug. Sulfamerazine belongs to the family of sulfonamide drugs, which are commonly used as antibacterial agents. Sulfamerazine has very limited solubility in water.

\subsection{Experimental Procedures}

\subsubsection{Materials}

Sulfamerazine were purchased from Sigma Aldrich (MO, USA). Zirconium oxide beads (0.5 $\mathrm{mm}$ diameter) were purchased from NextAdvance (NY, USA). Trifluoroacetic 
acid was purchased from Fisher Scientific (PA, USA). Polyvinylpyrrolidone (PVP) K30 was kindly donated by BASF (NJ, USA). De-ionized water was used in this study.

\subsubsection{Wet-bead milling}

Wet-bead milling was carried out using a Retsch Ball mill (Model MM200, PA, USA). The frequency of vibration was kept constant at $30 \mathrm{~Hz}$ and the milling time was maintained for 6 hours. One gram of Sulfamerazine, four grams of zirconium oxide beads, and five milliliters of milling medium were loaded into a $10 \mathrm{~mL}$ stainless steel jar. Milling mediums were PVP solutions ( $1 \% \mathrm{w} / \mathrm{v})$ with and without salts. Three types of salts were studied (sodium iodide, sodium chloride, and sodium sulfate) at three concentrations of $0.1 \mathrm{M}, 0.5 \mathrm{M}$, and $1 \mathrm{M}$.

\subsubsection{Particle size measurement}

Laser diffractometry (Micromeritics, GA, USA) with DigiSizer5200 software was employed to analyze particle size distribution. Sulfamerazine saturated solution was chosen as the background solution and powders were dispersed in this solution. Particle size was expressed as volume distribution according to the Mie theory. Relative refractive index of water (1.33) was included in the calculations used in this experiment.

\subsubsection{Determination of PVP adsorption on particle surface}

The amount of PVP adsorbed on particle surface was determined by subtracting the PVP amount left in the solution after milling from total PVP amount in the initial solution (initial solution is 1\% PVP solution: $50 \mathrm{mg}$ PVP in $5 \mathrm{~mL}$ milling medium (theoretical); $49.6 \mathrm{mg}$ PVP in $5 \mathrm{~mL}$ milling medium (actual)). All samples were first filtered through a $0.22 \mu \mathrm{m}$ membrane filter. A HPLC system (Shimadzu, Japan) which included a solvent delivery module, a vacuum degasser, a column oven, and a diode array detector was utilized to determine the amount of PVP in solutions. Aliquots of $10 \mu \mathrm{L}$ of filtered samples were injected into a reversed phase $C_{18}$ column $(4 \mu \mathrm{m}, 4.6 \times 150 \mathrm{~mm}$, Waters, Ireland). The column temperature was maintained at $35^{\circ} \mathrm{C}$. The mobile phase consisted of $0.01 \%(\mathrm{v} / \mathrm{v})$ trifluoroacetic acid in water was supplied at a flow rate of 0.6 $\mathrm{mL} / \mathrm{min}$. The wavelength for PVP quantification was set at $200 \mathrm{~nm}$.

\subsubsection{Lyophilization}

After 6-hours of ball milling, $5 \mathrm{~mL}$ of suspension was withdrawn from the jar and lyophilized at $-80^{\circ} \mathrm{C}$ and 0.01 mbar for 60 hours (Labconco, KS, USA). Nano-micro suspensions milled in medium without salt (Formulation 1) and in medium with $1 \mathrm{M}$ sodium sulfate (Formulation 2) were selected for lyophilization and further evaluation. 


\subsubsection{Determination of drug content in lyophilized powder blends}

To evaluate drug content in lyophilized powder blends, Sulfamerazine amounts were quantified at $263 \mathrm{~nm}$ using a UV spectrometer (PerkinElmer, MA, USA) with proper dilution. Briefly, $10 \mathrm{mg}$ of powder blends were dissolved in $100 \mathrm{~mL}$ of de-ionized water, followed by mechanical shaking overnight. Drug loading was characterized as the percentage of drug amount in the powder blend.

\subsubsection{Powder dissolution tests}

In-vitro powder dissolution tests were performed using a USP paddle apparatus (Hanson Research, CA, USA). Briefly, $25 \mathrm{mg}$ of Sulfamerazine powder or powder blend equivalents to drug content (lyophilized) were first dispersed in $2 \mathrm{~mL}$ of de-ionized water with manual shaking. This suspension was prepared in order to break any loose agglomerates.

Afterwards, the suspension was loaded to $500 \mathrm{~mL}$ of de-ionized water. The temperature was maintained at $37^{\circ} \mathrm{C}$. Paddle rotation speed was kept constant at $25 \mathrm{rpm}$. Powders of lyophilized Formulation 1 and Formulation 2 were evaluated for dissolution test and conventional Sulfamerazine was utilized as the control. The concentration of Sulfamerazine was monitored at $263 \mathrm{~nm}$ using a UV spectrophotometer. Three replicates were carried out for each sample.

\subsubsection{Differential scanning calorimetry}

DSC Q2000 (TA Instruments, New Castle, DE, USA) equipped with a nitrogen cooler that purge nitrogen gas at $50 \mathrm{~mL} / \mathrm{min}$ was utilized to explore the thermal behavior of the samples. The instrument was calibrated using Indium standard. Four samples (approximately $5 \mathrm{mg}$ each) were tested. These included sodium sulfate, conventional Sulfamerazine (crystalline), lyophilized powder of Formulation 1, and lyophilized powder of Formulation 2. Samples were first sealed in an Aluminum pan. Thereafter, samples were heated at $10{ }^{\circ} \mathrm{C} / \mathrm{min}$ from 25 to $250^{\circ} \mathrm{C}$.

\subsubsection{Stability tests}

Lyophilized powders from suspensions were stored at $25^{\circ} \mathrm{C} / 65 \% \mathrm{RH}$. Particle size distribution was analyzed again after one month according to parameters in Section 3.2.3. 


\subsection{Results}

\subsubsection{Particle size measurement}

It has been reported that wet bead milling is an effective method to reduce particle size to the nanometer size. ${ }^{57,58,59,60,61,62,63}$ In this study, the effect of the anions of various sodium salts in the suspension medium on wet milling efficiency was investigated; other important parameters such as milling frequency, milling time, amount of drug, amount of stabilizer PVP (K30), beads, and medium volume were kept constant. The average diameters $\left(\mathrm{d}_{(\mathrm{v}, 0.1)}, \mathrm{d}_{(\mathrm{v}, 0.5)}\right.$, and $\left.\mathrm{d}_{(\mathrm{v}, 0.9)}\right)$ for $10 \%, 50 \%$, and $90 \%$ of all measured particles after milling were plotted in Figure 3-1. Figure 3-1 shows particle diameters for milled suspensions without salts, and with $0.1 \mathrm{M}, 0.5 \mathrm{M}$ and $1 \mathrm{M}$ of $\mathrm{NaI}, \mathrm{NaCl}$ and $\mathrm{NaSO}_{4}$ respectively. Before milling, the particle size distribution of Sulfamerazine powders was in the micron range and hardly any particles were in nanometer range since $\mathrm{d}_{(\mathrm{v}, 0.1)}$ was $10.1 \pm 2.9 \mu \mathrm{m}, \mathrm{d}_{(\mathrm{v}, 0.5)}$ was $31.1 \pm 0.5 \mu \mathrm{m}$, and $\mathrm{d}_{(\mathrm{v}, 0.9)}$ was $84.1 \pm 2.4 \mu \mathrm{m}$. After milling, Sulfamerazine particle size was reduced significantly and the majority of particles $d(v, 0.9)$ was in the lower micron range. Since all the mediums used for milling contained $1 \%(\mathrm{w} / \mathrm{v})$ of PVP, the one which had no salt used as a control. After milling, the $\mathrm{d}_{(\mathrm{v}, 0.5)}$ was $1.665 \pm 0.101 \mu \mathrm{m}$ for this control and approximately $20.9 \%$ of total volume of powders were below $1 \mu \mathrm{m}$ (see first bar in Figure 3-1). The volume percentiles of particles with a diameter less than $1 \mu \mathrm{m}$ were plotted in Figure 3-1b. As can be seen in Figure 3-1b, the volume percentile for the majority of particle size distributions for the milled suspensions which contained sodium iodide and sodium chloride were below $30 \%$ including the $0.1 \mathrm{M}$ of sodium sulfate suspension. The student $\mathrm{t}$ test was utilized to analyze statistical significance, the $1 \mathrm{M}$ sodium iodide was not statistically significant as compared to control. The $1 \mathrm{M}$ sodium chloride was statistically significant as compared to control $(p<0.05)$ and the $1 \mathrm{M}$ sodium sulfate was more statistically significant $(\mathrm{p}<0.01)$.

Although this effect was not very obvious at sodium sulfate concentration of $0.1 \mathrm{M}$ which showed a $\mathrm{d}_{(\mathrm{v}, 0.5)}$ value of $1.653 \pm 0.024 \mu \mathrm{m}$ and a volume percentile of $21.2 \%$ for particles diameters below $1 \mu \mathrm{m}$, interestingly, the particle reduction became significant as sodium sulfate concentrations increased to $0.5 \mathrm{M}$ where $\left(\mathrm{d}_{(\mathrm{v}, 0.5)}\right.$ was $1.285 \pm 0.023 \mu \mathrm{m}$ and the volume percentiles of particles with a diameter below $1 \mu \mathrm{m}$ was $32.4 \%$. As the sulfate concentration increased to $1 \mathrm{M}$, the $\mathrm{d}_{(\mathrm{v}, 0.5)}$ became $0.934 \pm 0.015 \mu \mathrm{m}$ and the volume percentiles of particles with diameters below $1 \mu \mathrm{m}$ were $55.3 \%$. According to the classification of Van Eerdenbrugh and coworkers, the milled suspension with sodium sulfate $(1 \mathrm{M})$ is Nanosuspension since more than $50 \%$ of the volume of particles was below $1 \mu \mathrm{m}$ and $\mathrm{d}_{(\mathrm{v}, 0.9)}$ was below $2.5 \mu \mathrm{m} .^{62}$

A possible explanation for the effect of salts on particle size is related to the tested anion which can decrease the stabilizer's (PVP) solubility in water and consequently enhance the adsorption of the stabilizer onto the Sulfamerazine particle surface. Nanoparticles typically have higher surface energy as compared to the micron range particles and are not thermodynamically stable due to particle aggregation and/or crystal growth. To stabilize a nanosuspension, polymeric stabilizers are introduced to stabilize 


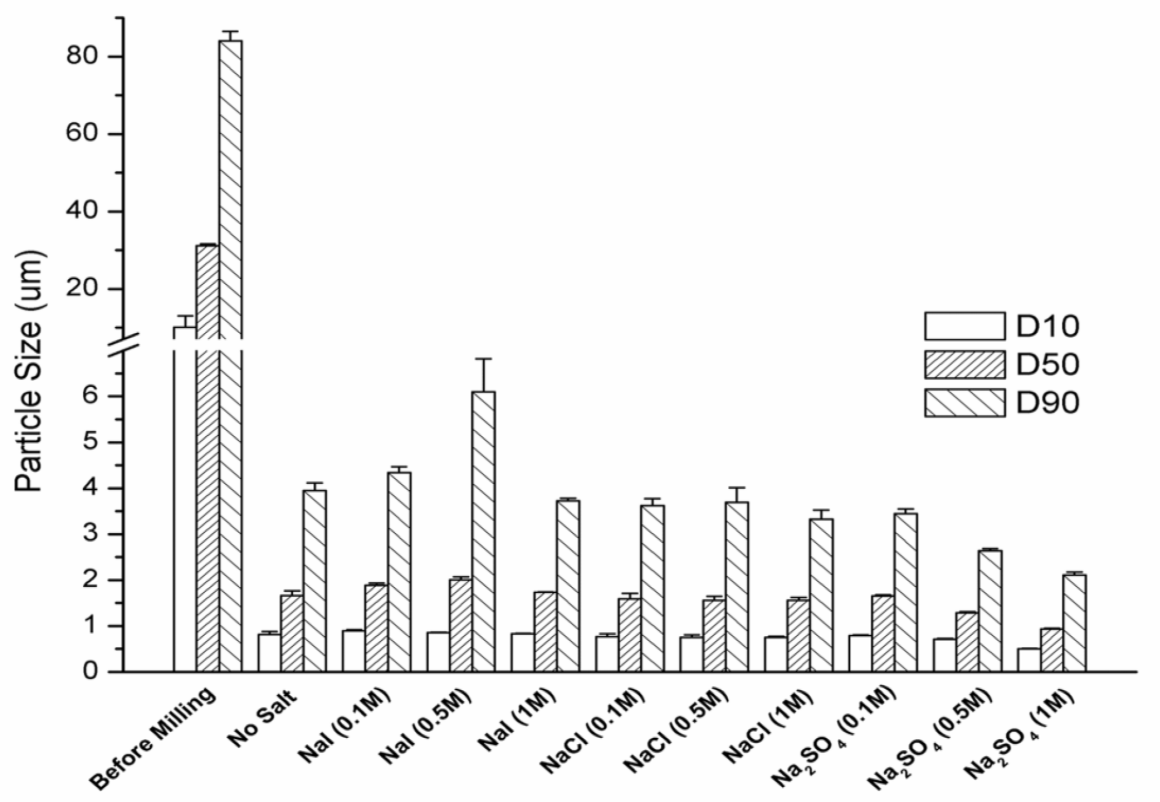

b

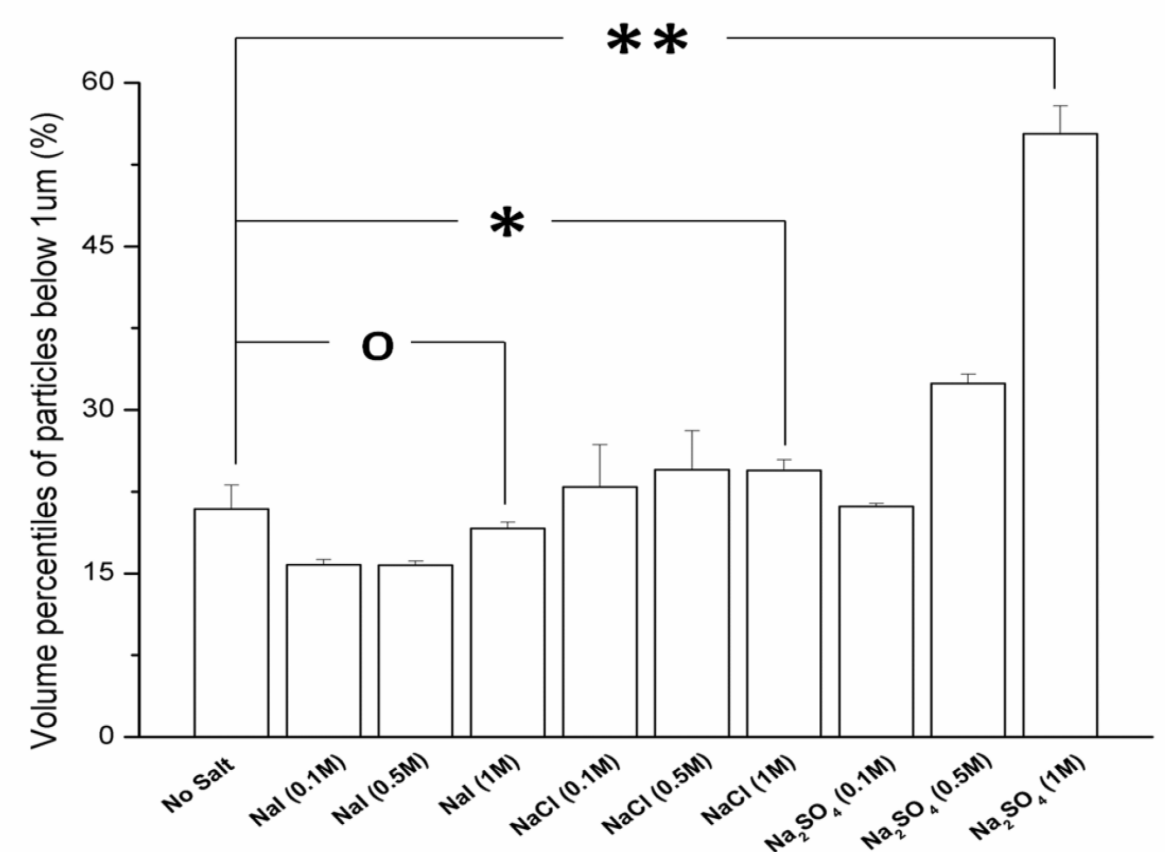

Figure 3-1. Particle size and volume percentiles of particles in suspensions below $1 \mu \mathrm{m}$.

$* *: \mathrm{p}<0.01 ; *: \mathrm{p}<0.05 ; \quad$ : no significant difference. 
the nanoparticles and one of the critical requirements for selection of a stabilizer is its ability to adsorb onto drug particle surface. ${ }^{71}$ Thus, we hypothesize that sulfate ions at relatively high concentrations increases the total amount of PVP adsorbed onto particle surface, thereby causing more stabilizing influence on nanoparticles stability and enhancing the milling efficiency. This effect can be rationalize according to Hofmeister ion series where the sulfate ion tends to break the water structure and influence the hydration on the Sulfamerazine particle consequently increasing the adsorption of PVP onto the particle surface. We have not considered conducting solubility of PVP in salt solutions because PVP is freely soluble in water and viscosity becomes a limitation at high concentrations.

\subsubsection{Determination of PVP adsorption on particle surface}

Several scientists illustrated that the adsorption of polymers onto solid surface is critical for nanoparticle stabilization by weak interactions such as steric forces. ${ }^{72}$ Ploehn and Russel referred to the adsorption of polymer as a compensation of free energy reduction over entropy loss. ${ }^{73}$ It has also been reported that anions in the Hofmeister series have important effect on polymers' solubility and adsorption onto solid surfaces. ${ }^{68,74}$ Therefore, the quantitative determination of polymer adsorption on particle surfaces is an important and efficient approach to validate this assumption. As we mentioned, the function of (PVP) during milling is the adsorption onto the surface of the drug particle (described in Section 3.3.1). The amounts of PVP adsorbed onto drug particle surface were calculated by measuring the depletion of PVP from the medium.

Adsorption data are listed in Table 3-1. As we observed, anions (sulfate, chloride, and iodide) have different influences on PVP adsorption. The total amount of adsorbed PVP for control (no salt) was $4.9 \pm 2.0 \mathrm{mg} / \mathrm{g}$ and $4.8 \pm 1.9 \mathrm{mg} / \mathrm{m}^{2}$. It was shown that as iodide salt was applied regardless of different concentrations, the total amount of adsorbed PVP was negligibly different to that without salt (control). On the other hand, chloride and sulfate salts increased PVP adsorption considerably, especially at higher concentrations; it was indicated that $12.5 \pm 2.5 \mathrm{mg} / \mathrm{g}$ PVP was adsorbed with sodium chloride $(1 \mathrm{M})$ and $20.2 \pm 0.8 \mathrm{mg} / \mathrm{g}$ PVP was adsorbed with sodium sulfate $(1 \mathrm{M})$ respectively. In view of this, we could lead to a conclusion about the rank of capability of anions' influence on PVP dehydration which is sulfate $>$ chloride $>$ iodide. Moreover, by tying these values to particle size values, it is worth suggesting that the stabilization of particle size in nanometer level might be partly due to more PVP which covers larger surface area of nanometers. Additionally, it is logically hypothesized that more PVP molecules consequently have higher steric interaction to stabilize the nanosuspension.

\subsubsection{Lyophilization}

Spray drying and lyophilization are currently the two most typical methods for drying suspensions. ${ }^{74}$ In this study, we anticipated the circulation of spray dryer would easily be clogged by viscous milled suspensions and therefore we decided not to use the 
Table 3-1. The amounts of PVP adsorbed onto Sulfamerazine particles after milling at concentrations.

\begin{tabular}{ccc}
\hline $\begin{array}{c}\text { Milling Medium } \\
\text { PVP Solution 1\% } \\
\text { (with or without Salt) }\end{array}$ & $\begin{array}{c}\text { PVP in } \\
\text { Solution after } \\
\text { Milling } \\
(\mathbf{m g})\end{array}$ & $\begin{array}{c}\text { PVP Adsorbed onto } \\
\text { Sulfamerazine } \\
\text { Particle Surface } \\
\text { (mg/g) }\end{array}$ \\
\hline No salt & $44.7 \pm 2.0$ & $4.9 \pm 2.0$ \\
Sodium Iodide 0.1 M & $45.2 \pm 1.3$ & $4.4 \pm 1.3$ \\
Sodium Iodide 0.5 M & $45.3 \pm 1.6$ & $4.3 \pm 1.6$ \\
Sodium Iodide 1 M & $44.2 \pm 0.7$ & $5.4 \pm 0.7$ \\
Sodium Chloride 0.1 M & $44.0 \pm 2.3$ & $5.6 \pm 2.3$ \\
Sodium Chloride 0.5 M & $39.3 \pm 2.8$ & $10.3 \pm 2.8$ \\
Sodium Chloride 1 M & $37.1 \pm 2.5$ & $12.5 \pm 2.5$ \\
Sodium Sulfate 0.1 M & $42.0 \pm 2.1$ & $7.6 \pm 2.1$ \\
Sodium Sulfate 0.5 M & $37.3 \pm 0.9$ & $12.3 \pm 0.9$ \\
Sodium Sulfate 1 M & $29.4 \pm 0.8$ & $20.2 \pm 0.8$ \\
\hline
\end{tabular}


spray dryer; instead, we removed water by lyophilization. We lyophilized two suspensions, the first suspension was milled in medium containing no salt (Formulation 1); and the second suspensions was milled in medium containing $1 \mathrm{M}$ sodium sulfate (Formulation 2) which exhibited the highest percentage of particles in nanometer size. After drying, loose agglomerates were observed in both powder blends. One explanation for this phenomenon is the existence of capillary forces during drying and which could be the factor of nanoparticle agglomeration. ${ }^{75}$ Moreover, particle size measurements indicated that these agglomerates were too loose and broke easily upon redispersion. As shown in Figure 3-2, there were no particle size changes for Formulation $1: \mathrm{d}_{(\mathrm{v}, 0.5)}=1.615 \pm 0.026 \mu \mathrm{m}$ and the volume percentiles of particles with a diameter below $1 \mu \mathrm{m}$ was $20.8 \%$ (after lyophilization); $\mathrm{d}_{(\mathrm{v}, 0.5)}: 1.665 \pm 0.101 \mu \mathrm{m}$; the volume percentiles of particles with a diameter below $1 \mu \mathrm{m}: 20.9 \%$ (before lyophilization). On the other hand, for Formulation 2, after lyophilization, particle size $\left(\mathrm{d}_{(\mathrm{v}, 0.5)}: 1.085 \pm 0.127 \mu \mathrm{m}\right.$; the volume percentiles of particles with a diameter below $1 \mu \mathrm{m}: 45.5 \%$ ) increased slightly as compared to that before lyophilization. Despite a slight increase of particle size during drying, particle size of dried powders from suspension Formulation 2 was significantly smaller than that of dried powders from suspension Formulation 1.In addition, percentage of drug content in lyophilized powder blends was determined. Values are summarized as follows: $95.6 \pm 4.4 \%$ for the sample obtained from the suspension Formulation 1 and $59.4 \pm 3.7 \%$ for the sample obtained from the suspension Formulation 2. Student $t$ test shows no significant difference of particle percentile below $1 \mu \mathrm{m}$ for lyophilized Formulations 1 and 2 and lyophilized Formulations 1 and 2 after 1 month ( $>0.05)$.

\subsubsection{Powder dissolution tests}

Van Eerdenbrugh and co-workers indicated that nanoparticle agglomeration occurs almost inevitably during drying with no addition of matrix former. ${ }^{74}$ Therefore, we strive to preserve the nanoparticles in loose agglomerates that can break up easily. The lyophilized powders of suspension Formulation 1 and suspension Formulation 2 deaggromerated in water via manual shaking, therefore, all powders were first re-dispersed to form suspensions and then dissolution evaluation was performed for powders in the suspended state. Moreover, the re-dispersed nanosuspension may be the ideal formulation for dosing patients for two reasons: (1) nanoparticle advantages can be retained; (2) this formulation can be prepared easily by patients at home on their own.

The dissolution profiles of conventional crystalline Sulfamerazine powder, the lyophilized suspension Formulation 1 (control), and the lyophilized suspension Formulation 2 (1 M sodium sulfate) are plotted in Figure 3-3. Typically, particles in suspensions dissolve faster than dry powders, especially for hydrophobic materials, since wetting process takes time. As expected, the lyophilized Formulation 1 released $86 \%$ of Sulfamerazine within 5 minutes while the lyophilized Formulation 2 released $95 \%$ of Sulfamerazine within 5 minutes. Both profiles were significantly faster as compared to conventional crystalline Sulfamerazine powder which $67 \%$ had released within 5 minutes.

This indicates that wet bead milling is an effective method for dissolution rate 


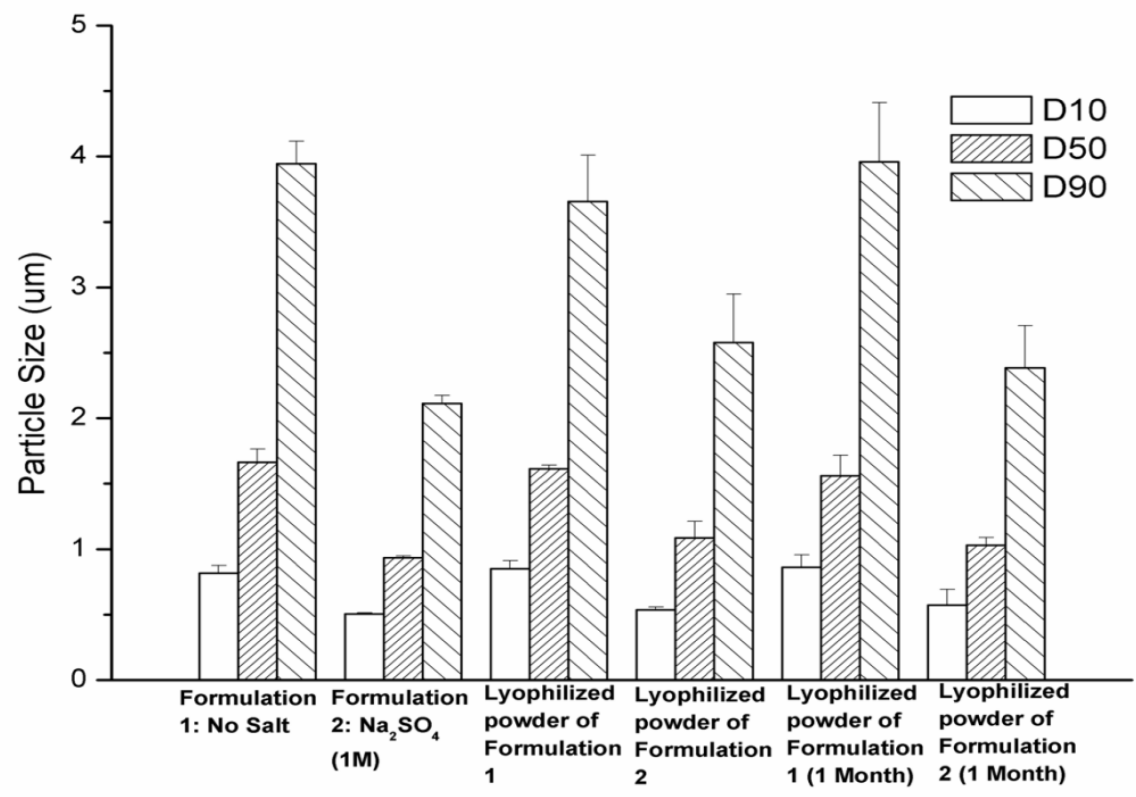

b

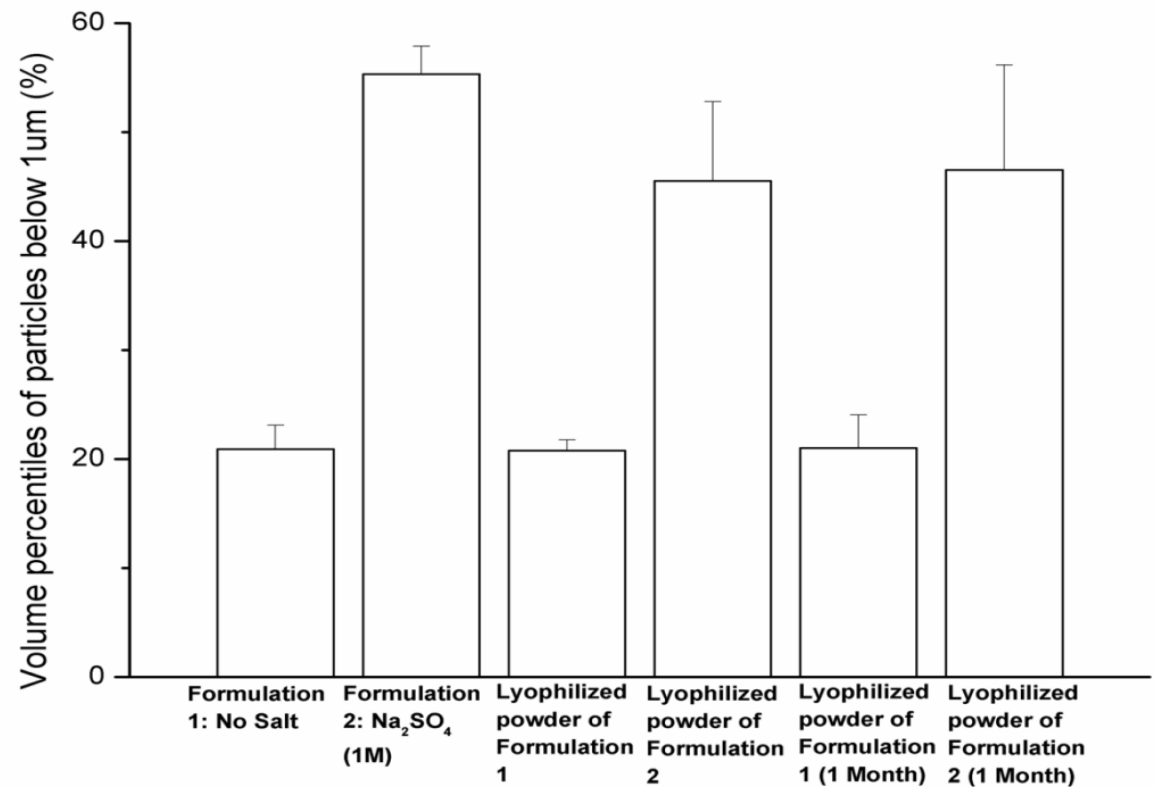

Figure 3-2. Particle size and volume percentiles of particles below $1 \mu \mathrm{m}$ of lyophilized formulations. 


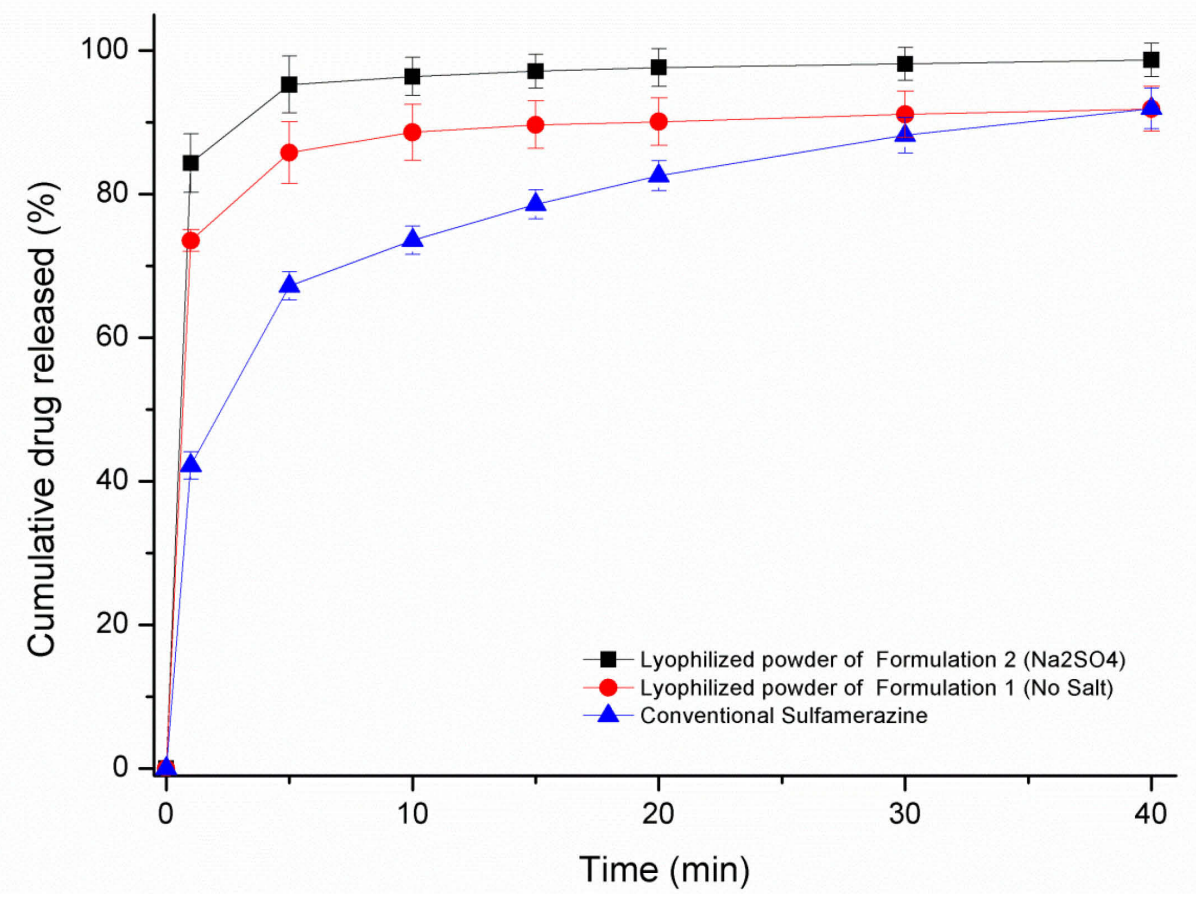

Figure 3-3. Dissolution profiles of Sufamerazine. 
enhancement of Sulfamerazine. In addition, the difference of dissolution rate between powder of lyophilized Formulation 2 and powder of lyophilized Formulation 1 is relatively small but still distinguishable. This difference partially attributed to the fact that powders of lyophilized Formulation 2 had smaller particle size. Although this dissolution tests provided a sound validation to the assumption that nanocrystals leaded to higher dissolution rate, the correlation between dissolution and particle size could not be established here.

\subsubsection{Differential scanning calorimetry}

DSC is utilized to characterize crystalline states and phase transitions of solids. It has been reported that Sulfamerazine has two polymorphs (I and II) and these two polymorphs are enantiotropic polymorphs. ${ }^{76}$ Zhang et al also revealed that polymorph II transforms into polymorph I at $173-175^{\circ} \mathrm{C}$ with an endothermic peak, and then polymorph I shows a significant endothermic peak and melts at around $240{ }^{\circ} \mathrm{C} .{ }^{76}$ In this experiment, to confirm whether there is a phase transformation of Sulfamerazine during milling and lyophilization, lyophilized samples were evaluated by DSC. All DSC thermograms are shown in Figure 3-4. There was no phase change identified for sodium sulfate in the studied range. Clearly, the DSC thermograms indicate that the conventional Sulfamerazine exists as polymorph I since it indicates only an endothermic pick at around $238{ }^{\circ} \mathrm{C} .{ }^{76}$ In addition, both powders of lyophilized Formulation 1 and powders of lyophilized Formulation 2 have very similar thermal behavior as conventional Sulfamerazine since all samples melted at 235 to $238^{\circ} \mathrm{C}$. Therefore, both powders of lyophilized Formulation 1 and lyophilized Formulation 2 exist as polymorph I. We can conclude that no polymorphic phase transformations were observed during milling and lyophilization processes.

\subsubsection{Stability}

Agglomerates could be observed in powder blends before and after stability tests. These agglomerates could be removed via dispersing in a solution with shaking. Particle size analysis revealed that there was no considerable change in particle size distribution before samples which underwent 1-month stability test and fresh samples, as shown in Figure 3-2.

\subsection{Discussion}

Polyvinylpyrrolidone (PVP), a commonly-used stabilizer, was chosen and kept constant in this study. Our intention is to investigate the influence of various anions in Hofmeister series on wet milling. Salts such as sodium sulfate played a significant role on increasing nano-microcrystal milling efficiency and were kept in lyophilized powder blend. Therefore, the percentage of excipient (excipient loading) in powder blend is worth to be noted. In lyophilized Formulation 2 powder blend, percentage of sodium 


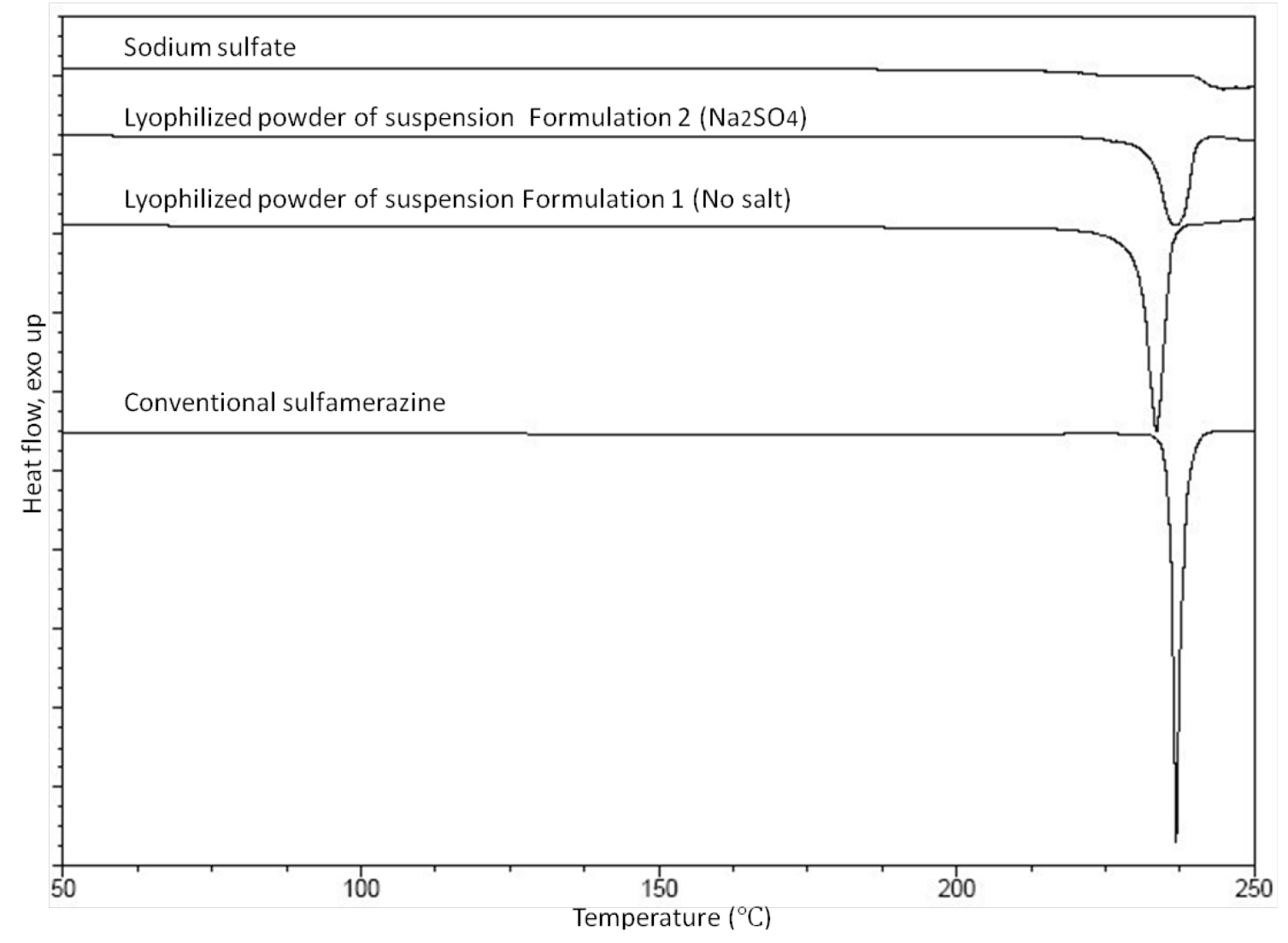

Figure 3-4. DSC patterns of various powder samples. 
sulfate was around 39\% (w/w) and PVP was less than 3\% (w/w). In other words, while $167 \mathrm{mg}$ of Sulfamerazine needs to be dosed followed by a prescription using this formulation, approximately $110 \mathrm{mg}$ of sodium sulfate in the blend was also dosed. It is necessary to point out that, at this dose level of sulfate, no considerable adverse effects were observed and reported in previous human and animal studies. ${ }^{77}$ Additionally, this study is a representative example of expanding a new application of Hofmeister since series salts. Sodium sulfate, in this study, had a "salting-out" effect, which lead to an increase of milling efficiency (Formulation 2 with $1 \mathrm{M}$ sodium sulfate, $55.5 \%$ of particles less than $1 \mu \mathrm{m}$ vs. Formulation 1 without salt, $20.9 \%$ of particles less than $1 \mu \mathrm{m}$ ). Oppositely, sometimes "salting-in" effect is pursued and applied. For example, more organic solvent can be miscible with aqueous phase with the aid of "salting-in" effect. In this scenario, some anions ranking at the right side of Hofmeister series such as Iodide may be considered. Since salts in Hofmeister series influence the alignment and quantity of hydrocarbons including various amphiphiles at different interfaces (e.g. liquid/liquid, liquid/solid, liquid/gas), the potential expansion of its pharmaceutical application is quite promising and may encompass multiple fields including liquid formulation production (e.g. emulsion, suspension, gel, cosolvent, micelle, protein/peptide formulations) and solid dosage form development (e.g. coating, wet milling). This study also indicated that nonocrystallization is an effective and stable method for dissolution rate enhancement of poorly-water soluble drugs. Moreover, most excipients that enhance the production efficiency of nanocrystals are cost-economical and therefore have the potential feasibility for large-batch size utilization. Despite the fact that considerable percentage of excipients in lyophilized powder blend, drug loading in this product is still generally higher than another prevalent drug dissolution enhancement approach: amorphous solid dispersion, in which large amounts of excipients are usually necessitated to stabilize drug in amorphous state. $^{78,79,80}$ In light of dissolution enhancement, a new concept which combines these two techniques has been developed: amorphous drug nanoparticles. Amorphous drug nanoparticles can be produced by some specific "bottom-up" methods, similar as nanocrystal "bottom-up" methodology, and eventually dispersed in aqueous phase as an amorphous nanosuspension. This novel nano-level formulation, may offer an even higher saturated solubility and faster dissolution rate as compared to regular nanocrystals. ${ }^{81}$ Unfortunately, instability is a challenging obstacle for this technique's maturation. As a consequence, in amorphous nanosuspension system, it is very likely to form small amounts of nucleus and/or crystalline particles, and then crystals keep growing as amorphous nanoparticles dissolve simultaneously, ${ }^{81}$ which results in the lost of solubility/dissolution advantage.

\subsection{Conclusion}

In this study, it was shown that wet bead milling is a successful approach to reduce particle size of Sulfamerazine. Sodium sulfate $(1 \mathrm{M})$ in milling medium could enhance milling efficiency of drug particles significantly and a higher percentage of particles were in nanometer level but other salts had minimal effects compared to control. Furthermore, powders after milling and lyophilization processes showed remarkable dissolution rate improvement. 


\section{CHAPTER 4. DEVELOPMENT OF A COATED MINI-TABLET OF CO- GRINDED PREDNISONE-NEUSILIN COMPLEX FOR PEDIATRIC USE *}

\subsection{Introduction}

Due to the lack of pediatric formulations, the regulatory agencies have initiated continual emphasis on the necessity for developing novel pediatric formulations. In response, pharmaceutical companies have developed various novel pediatric formulations some of which have already been launched into the market. ${ }^{1}$

In general, oral delivery of pediatric drugs is preferred over other delivery routes since it is convenient, economical, and user-friendly. ${ }^{1}$ In this study, we utilized Prednisone as a model drug and provided formulation methods for developing an oral solid dosage form that can be compatible with pediatric patients. Prednisone is a glucocorticoid prodrug which converts to its active metabolite Prednisolone in the liver. ${ }^{82}$ For decades, this drug has been involved widely in pediatric therapies. At low doses, Prednisone is involved in the treatment of emergency rescues such as acute asthma. ${ }^{83} \mathrm{At}$ relatively high doses, Prednisone is prescribed for the treatment of pediatric cancer. ${ }^{84}$ Although Prednisone has therapeutic uses for many pediatric diseases, there are several challenges that should be addressed with the oral delivery of this drug. These challenges include: (1) this drug is sparingly soluble in water and has low bioavailability; any liquid formulation must include organic solvents; (2) this drug has low tolerance depending on the dose strength and can cause fluctuation in overall absorption; (3) this drug should be in a dosage form that could be swallowed readily; (4) this drug is extremely bitter which requires better palatability through taste masking.

To improve the dissolution rate of poorly-soluble drugs, a number of approaches have been reported including amorphilization, ${ }^{49}$ nanocrystal, ${ }^{55,85,86}$ complexation, ${ }^{51}$ salt formation, ${ }^{52}$ and polymorph transformation. ${ }^{50}$ Although an amorphous form dissolves faster than a crystalline form, amorphous solids retain higher free energy levels which cause the solid to become unstable and inherit the tendency to convert to the crystalline form. In order to stabilize amorphous molecules during the storage and dissolution process, preparing solid dispersion is one approach and various polymers such as PVP, PEG or cellulose derivatives have been widely used as dispersants. ${ }^{87,88}$ In contrast to organic polymers, Neusilin can also function as a carrier to keep drug in amorphous state. ${ }^{89,90}$ Neusilin ${ }^{\circledR}$ US2 is a porous granule of amorphous magnesium aluminosilicate which retains a tremendous surface area (around $300 \mathrm{~m}^{2} / \mathrm{g}$ ). The surface of Neusilin contains a large amount of silanol functional groups which facilitate the formation of hydrogen bonds. ${ }^{91,92,93}$ After cogrinding a drug with Neusilin ${ }^{\circledR}$ US2, an amorphous drugNeusilin complex is formed. ${ }^{89,90,94}$ One possible mechanism for the formation of

* : Adapted with permission. Lou H, Liu M, Wang L, Mishra SR, Qu W, Johnson J, Brunson E, Almoazen H. Development of a mini-tablet of co-grinded prednisone-neusilin complex for pediatric use. AAPS PharmSciTech. In Press. 
amorphous complex is linked to the crystalline structure disruption which is caused by force of mechanical shearing during milling then followed by the adherence of amorphous pieces into Nuesilin ${ }^{\circledR}$ US2 surfaces by hydrogen bonds or salt bridge formation. ${ }^{91}$ It is well known that Neusilin ${ }^{\circledR}$ US2 is involved in solid dosage form not only as an adsorbent but also as a low-toxicity excipient which can increase tablet hardness. ${ }^{95}$

Swallowing ability is critical for pediatric formulations. Regular solid dosage forms such as capsules or tablets are unfortunately not suitable for pediatric population since essentially children less than 10-year-old are not able to grasp the skills of swallowing regular size tablets or capsules. Although granules and powders are suitable replacements for tablets or capsules, particle size must be characterized to prevent any dose fluctuations. To achieve swallowing appropriateness and good unit dose strength, mini-tablet is a suitable candidate. Mini-tablet is defined as a tablet with a diameter less than $3 \mathrm{~mm} .{ }^{13}$ A recent study conducted by Thomson et al. illustrated that out of 100 preschoolers, no one suffered the potential of choke caused by swallowing placebo minitablets and up to $86 \%$ of 5-year-old children swallowed mini-tablets successfully. ${ }^{14}$ Additionally, the manufacturing procedures of mini-tablets can be scaled-up since minitablets can be compressed by regular tablet press with reproducible sizes, weights, and dose strengths. ${ }^{15}$

If a pharmaceutical compound or an excipient has poor pediatric compliance due to unpleasant taste, film coating with a polymer is a realistic solution. For instance, Eudragit ${ }^{\circledR}$ EPO is $\mathrm{s}$ a taste-masking and moisture protective material which has been extensively applied in film coating. ${ }^{29}$ Since it is a $\mathrm{pH}$-dependent copolymer, it is only slightly swellable when the $\mathrm{pH}$ is higher than 5 and is completely soluble when the $\mathrm{pH}$ is lower than 5. Therefore, Eudragit ${ }^{\circledR}$ EPO film coating can be used to achieve taste masking in the oral cavity $(\mathrm{pH}=6.8)$ while keeping the immediate release characteristics for the dosage form in stomach $(\mathrm{pH}=1.2)$.

The objective of this study was to develop a novel pediatric formulation for Prednisone. In this study, dissolution rate of Prednisone was enhanced after cogrinding Prednisone and Neusilin to form an amorphous complex. Moreover, this complex was compressed with other excipients into $2 \mathrm{~mm}$ diameter mini-tablets. Lastly, Eudragit ${ }^{\circledR}$ EPO multiple layers were coated on the mini-tablet for taste-masking purpose.

\subsection{Experimental Procedures}

\subsubsection{Materials}

Prednisone was purchased from Sigma Aldrich (MO, USA). Neusilin ${ }^{\circledR}$ US2 was kindly donated by Fuji Chemicals (NJ, USA). Eudragit ${ }^{\circledR}$ EPO is poly (butyl methacylateco-(2-dimethylaminoethyl) methacrylate-co-methyl methacrylate) and was kindly donated by Evonik (NJ, USA). Croscarmellose sodium was kindly donated by FMC 
Biopolymer (PA, USA). Magnesium stearate was kindly donated by Mallinckrodt (MO, USA). Silicified microcrystalline cellulose was kindly donated by JRS Pharma (NY, USA).

\subsubsection{Milling}

A Retsch Ball mill (Model MM200, PA, USA), attached with a $25 \mathrm{~mL}$ stainless steel jar and $5 \mathrm{~mm}$ diameter stainless steel ball, was used to grind Prednisone with and without Neusilin ${ }^{\circledR}$ US2. The frequency of vibration was kept constant at $4 \mathrm{~Hz}$ and the milling operation time was maintained for 90 minutes. A 1 gram of Prednisone with variable amounts of Neusilin ${ }^{\circledR}$ US2 at various ratios $(1: 1,1: 3,1: 5$, and 1:7) were physically mixed and then milled at room temperature. After ball milling, samples were transferred to a loosely capped glass vial, and stored in vacuum at room temperature.

\subsubsection{Differential scanning calorimetry studies}

The thermo behavior of samples were explored using a DSC Q2000 (TA Instruments, New Castle, DE, USA) which is equipped with a nitrogen cooler at $50 \mathrm{~mL} / \mathrm{min}$ nitrogen purge. The instrument was calibrated using Indium standard. Seven samples (about $5 \mathrm{mg}$ each) were tested including Prednisone, Neusilin ${ }^{\circledR}$ US2, 1:1 Prednisone/Neusilin ${ }^{\circledR}$ US2 physical mixture, and co-ground 1:1, 1:3, 1:5: and 1:7 Prednisone/Neusilin ${ }^{\circledR}$ US2 complex. Samples were first sealed in an Aluminum pan and held at $40{ }^{\circ} \mathrm{C}$ for 2 minutes. Thereafter, samples underwent modulated heating rate at $5{ }^{\circ} \mathrm{C} /$ min associated with $0.5^{\circ} \mathrm{C}$ modulation amplitude every 40 seconds. The overall temperature scan ranged from 40 to $300^{\circ} \mathrm{C}$.

\subsubsection{X-ray powder diffraction and percent crystallinity}

The X-ray powder diffraction of Prednisone, Neusilin and co-ground Prednisone/Neusilin complexes at different ratios were obtained using Bruker AXS D8 advance diffractometer (Bruker, Germany) which is equipped with a copper anode (target, $\mathrm{Cu} \mathrm{K} \alpha$ radiation; voltage, $40 \mathrm{kV}$; current, $30 \mathrm{~mA}$; divergence slit, $3.722 \mathrm{~mm}$; antiscatter slit, $3.722 \mathrm{~mm}$; detector slit, $5 \mathrm{~mm}$; Goniometer radius, $300 \mathrm{~mm}$ ) at ambient temperature. A thin layer of powder sample was first laid on a sample holder and then measured at $2 \theta$ in a continuous range from $3^{\circ}$ to $40^{\circ}$ with a step size 0.048 . The percent crystallinity of samples was determined using Eva Software (Bruker). A two phase model was employed in the determination of the degree of crystallinity; that is the sample is composed of crystals and amorphous and no regions of semi-crystalline organization. Thus, the diffraction profile was divided in 2 parts: peaks related to diffraction of crystallites and broad region related to the scattering from amorphous phase. The assumption is that the areas are proportional to the scattering intensities of crystalline and amorphous phases. The percent crystallinity was calculated from the integrated intensities of all crystalline peaks and the amorphous region under the diffraction curve. Percent crystallinity of 
sample is defined as $\mathrm{I}_{\text {crystalline }} /\left(\mathrm{I}_{\text {crystalline }}+\mathrm{I}_{\text {amorphous }}\right)$. The contribution to the intensity from the amorphous region in the sample was identified by properly adjusting the curvature of the background curve. The contribution to the intensity from the crystalline region was obtained after subtracting the above background curve from the XRD pattern.

\subsubsection{Solubility measurements}

Excess amounts of Prednisone or co-ground Prednisone/Neusilin ${ }^{\circledR}$ US2 complex (1:7) were dispersed into $30 \mathrm{~mL}$ of simulated gastric fluid. The suspensions were placed in a mechanical shaker at a constant shaking rate of 50rpm and a constant temperature of $25^{\circ} \mathrm{C}$. Two milliliters of liquid were withdrawn at each selected time point, filtered through a $0.22 \mu \mathrm{m}$ membrane filter, and diluted to an appropriate ratio using simulated gastric fluid. The concentration of Prednisone was determined at $242 \mathrm{~nm}$ using UV spectrometer (PerkinElmer, MA, USA). Three replicates were carried out for each sample.

\subsubsection{Particle size measurement}

Laser diffractometry (Micromeritics, GA, USA) was employed to analyze particle size distribution. Saturated solution of Prednisone was chosen as the background solution. Particle size measurement of suspension containing excess powder blend was performed. Three replicates were performed for each sample. Particle size was expressed as volume distribution according to the Mie theory. Relative refractive index of water (1.33) was inserted in this experiment.

\subsubsection{Surface area measurement}

The specific surface area of each sample was determined by the gas adsorption method according to the BET theory using "Gemini" surface area analyzer (Micromeritics, GA, USA). Nitrogen gas was adsorbed to powder surface, and the amount adsorbed gas was converted into the surface area of powder. Analysis was performed at five relative pressures ranging from 0.05 to 0.2 .

\subsubsection{Morphology}

Morphologies of Prednisone, Neusilin, and co-ground 1:7 Prednisone/Neuislin, were characterized using the Environmental Scanning Electron Microscope (Philips, Netherland) equipped with a Peltier stage. Prior to microscope observation, all samples underwent platinum sputter-coating in argon. 


\subsubsection{Core mini-tablet preparation}

Three core mini-tablet formulations were prepared. Formulation 1 was directly compressed from a powder blend of (75.2\%) co-ground 1:7 Prednisone/Neusilin complex containing (9.4\%) Prednisone, (18.8\%) silicified microcrystalline cellulose, $(5 \%)$ croscarmellose sodium, and (1\%) magnesium stearate. Formulations 2 and 3 were prepared as control groups. In Formulation 2, drug and excipients were identical to Formulation 1, except for the use of 1:7 Prednisone/Neusilin physical mixtures. In Formulation 3, no Neusilin granules were added; thus (9.4\%) Prednisone was blended and compressed with (84.6\%) silicified microcrystalline cellulose, $(5 \%)$ croscarmellose sodium, and (1\%) magnesium stearate. All mini-tablets were compressed into a biconvex tablet with a diameter of $2 \mathrm{~mm}$ using a Carver hydraulic press (Carver INC, IN, USA). The compression force was kept constant at $125 \mathrm{lb}$.

\subsubsection{Characterization of core mini-tablets}

\subsubsection{Thickness, weight variation and unit dose strength}

Thickness of ten core mini-tablets for each formulation (Formulation 1,2 and 3) was determined using a vernier capiler. Weight variation was evaluated by weighing mini-tablets $(n=10)$.

To evaluate unit dose strength, ten core mini-tablets of each formulation were also utilized. Briefly, each mini-tablet was dissolved in $25 \mathrm{~mL}$ of solution containing water $(68.8 \%)$, tetrahydrofuran $(25.0 \%)$, and methanol (6.2\%), followed by mechanical shaking overnight to dissolve all Prednisone. The suspension was then filtered through a $0.45 \mu \mathrm{m}$ filter membrane, and the concentration of Prednisone was determined at $242 \mathrm{~nm}$ using UV spectrophotometer.

\subsubsection{Tensile strength and friability}

Tensile strength and friability of mini-tablets (Formulation 1) were conducted. Tensile strength was calculated using the following Equation 4-1:

$$
\text { Tensile strength }=\frac{2 * \mathrm{~F}_{\mathrm{d}}}{\pi * \mathrm{D} * \mathrm{H}}
$$

Where $\mathrm{F}_{\mathrm{d}}$ is tablet hardness, $\mathrm{D}$ is tablet diameter, and $\mathrm{H}$ is tablet central cylinder thickness. Tablet hardness was determined using a manual tablet testing instrument (Vortex sales group, NC, USA).

Friability tests were conducted using a friabilator (Sotax, MA, USA). Twenty mini-tablets were first de-dusted, weighed, and laid into the friabilator drum with around 6 gram of $3 \mathrm{~mm}$ glass beads. One hundred revolutions were set. Friability was calculated according to the following Equation 4-2. 


$$
\text { Percent Friability }=\frac{\text { initial weight }- \text { final weight }}{\text { initial weight }} \times 100 \%
$$

\subsubsection{Core mini-tablet coating}

Due to the small mini-tablet size, mini-tablets could not be coated by fluid bed or pan coating. Alternatively, dip-coating process was utilized to coat mini-tablets. A coating solution consisted of Eudragit $\AA$ EPO dissolved in acetone $(20 \%$, w/w) was utilized for coating. The mini-tablets (Formulation 1) were dipped in Eudragit ${ }^{\circledR}$ EPO solution for approximately 40 seconds and then air dried. Thereafter, a 15-second dipcoating process followed by air-drying was repeated 8 times. This group of coated minitablets was defined as Formulation 4. All coated mini-tablets were dried and stored in a vacuum oven at room temperature.

\subsubsection{Physical stability tests}

The purpose of this study is to examine the influence of moisture on the physical stability of Prednisone in the amorphous state over 4 week period. In this experiment, core mini-tablets (Formulation 1) and coated mini-tablets (Formulation 4) were stored in isolated desiccators at $75 \% \mathrm{RH}$ (supersaturated sodium chloride solution) in a $25{ }^{\circ} \mathrm{C}$ oven.

\subsubsection{Powder and mini-tablet dissolution tests}

In-vitro powder dissolution tests were performed using the USP paddle apparatus (Distek INC, NJ, USA). Five hundred milliliters of simulated gastric fluid (sodium chloride $2 \mathrm{~g} / \mathrm{L}$; $\mathrm{pH}$ adjusted to 1.2 with hydrochloric acid) was maintained at $37{ }^{\circ} \mathrm{C}$. Paddle rotation speed was kept constant at $50 \mathrm{rpm}$. Briefly, $10 \mathrm{mg}$ of Prednisone powder or drug content equivalent to Prednisone/Neusilin complex was uniformly sprinkled to the surface of the dissolution medium. Co-ground Prednisone/Neusilin complexes at different ratios were evaluated and conventional Prednisone was utilized as the control group. The concentration of Prednisone was monitored at $242 \mathrm{~nm}$ using an in-line fiber optic cable connected to ultraviolet detector (pION, MA, USA). Three replicates were carried out for each formulation.

Dissolution test parameters of core mini-tablets were kept constant as previous powder dissolutions. Eighteen mini-tablets (equivalent to $10 \mathrm{mg}$ of Prednisone) were directly dropped into the dissolution vessel. Three formulations (Formulations 1, 2, and 3) were evaluated and three replicates were carried out for each formulation.

Dissolution behavior of coated mini-tablets (Formulation 4) was evaluated in simulated gastric fluid $(\mathrm{pH}=1.2)$ as well as in simulated saliva $(\mathrm{pH}=6.8)$. In addition, dissolution rates of mini-tablets (Formulation 1 and Formulation 4) after stability test were evaluated in gastric fluid $(\mathrm{pH}=1.2)$. Dissolution parameters (temperature, paddle rotation speed, medium volume, and number of tablets) were kept identical to those of 
core mini-tablets. Three replicates were carried out for each formulation.

The dissolution profile similarity factor $\left(\mathrm{f}_{2}\right)$, as shown in the following Equation 4-3, was utilized to compare the dissolution profiles of mini-tablets before stability test in Section 4.2.12 and after stability test.

$$
\mathrm{f}_{2}=50 \times \log \left\{\left[1+(1 / \mathrm{n}) \sum_{t=1}^{n}\left(\mathrm{R}_{\mathrm{t}}-\mathrm{T}_{\mathrm{t}}\right)^{2}\right]^{-0.5} \times 100\right\}
$$

Where (n) is the number of time points (10 time points, over 60 minutes), $R_{t}$ is the mean percentage of drug released for $1^{\text {st }}$ curve at $t_{t}$ time point, $T_{t}$ is the mean percentage of drug release for $2^{\text {nd }}$ curve at $t_{t}$ time point. Two dissolution profiles are considered similar if $\mathrm{f}_{2} \geq 50$.

\subsection{Results and Discussion}

\subsubsection{Differential scanning calorimetry studies}

DSC is utilized to evaluate thermal behavior and phase transitions of solids. It provides useful information about the solid state properties (crystalline or amorphous) of solid materials. All DSC thermograms are shown in Figure 4-1. Conventional Prednisone thermogram shows an endothermic sharp peak at $235^{\circ} \mathrm{C}$. This refers to Prednisone melting point and also suggests that Prednisone is in crystalline state. On the other hand, Neusilin has no melting peak which is indicative of its amorphous state. Previous reports in the literature showed that mixing Neusilin with volatile crystalline organic compounds may transform these compounds from crystalline state into amorphous state. ${ }^{96}$ However, the 1:1 physical mixture of Prednisone and Neusilin did not show considerable phase transformation. Actually, the 1:1 Prednisone/Neusilin physical mixture showed an endothermic peak at around $225^{\circ} \mathrm{C}$ which indicates that Prednisone maintained some crystallinity. We also noticed a shift in melting peaks for 1:1 physical mixture and co-ground. This melting point reduction is linked to the interaction between Prednisone and Neusilin. In a physical mixture, the addition of Neusilin attracts Prednisone crystal aggregates and slightly lowers the melting point of Prednisone. In a co-ground mixture, Neusilin and Prednisone have closer interaction since some of the aggregates may have smaller size due to grinding and therefore Prednisone melting peak shifts to a lower melting point. Hence, the heat of melting of 1:1 physical mixture of Prednisone and Neusilin was $31.13 \mathrm{~J} / \mathrm{g}$ and decreased to $25.13 \mathrm{~J} / \mathrm{g}$ for $1: 1$ co-ground mixture. This suggests a small reduction in crystallinity. It is likely that only a certain amount of Prednisone could exist in amorphous state at 1:1 ratio, and the rest exists as small crystals after milling due to insufficient Neusilin adsorption surface. As the amount of Neusilin increases, the DSC curves of 1:3, 1:5, and 1:7 complexes show a complete disappearance of Prednisone endothermic peak. Thus, further addition of Neusilin caused major reduction in drug crystallinity. It was observed that when the content of Neusilin became three times in magnitude the drug content or even higher (co-ground 1:3

Prednisone/Neusilin complex), the majority of Prednisone stays in amorphous state. 


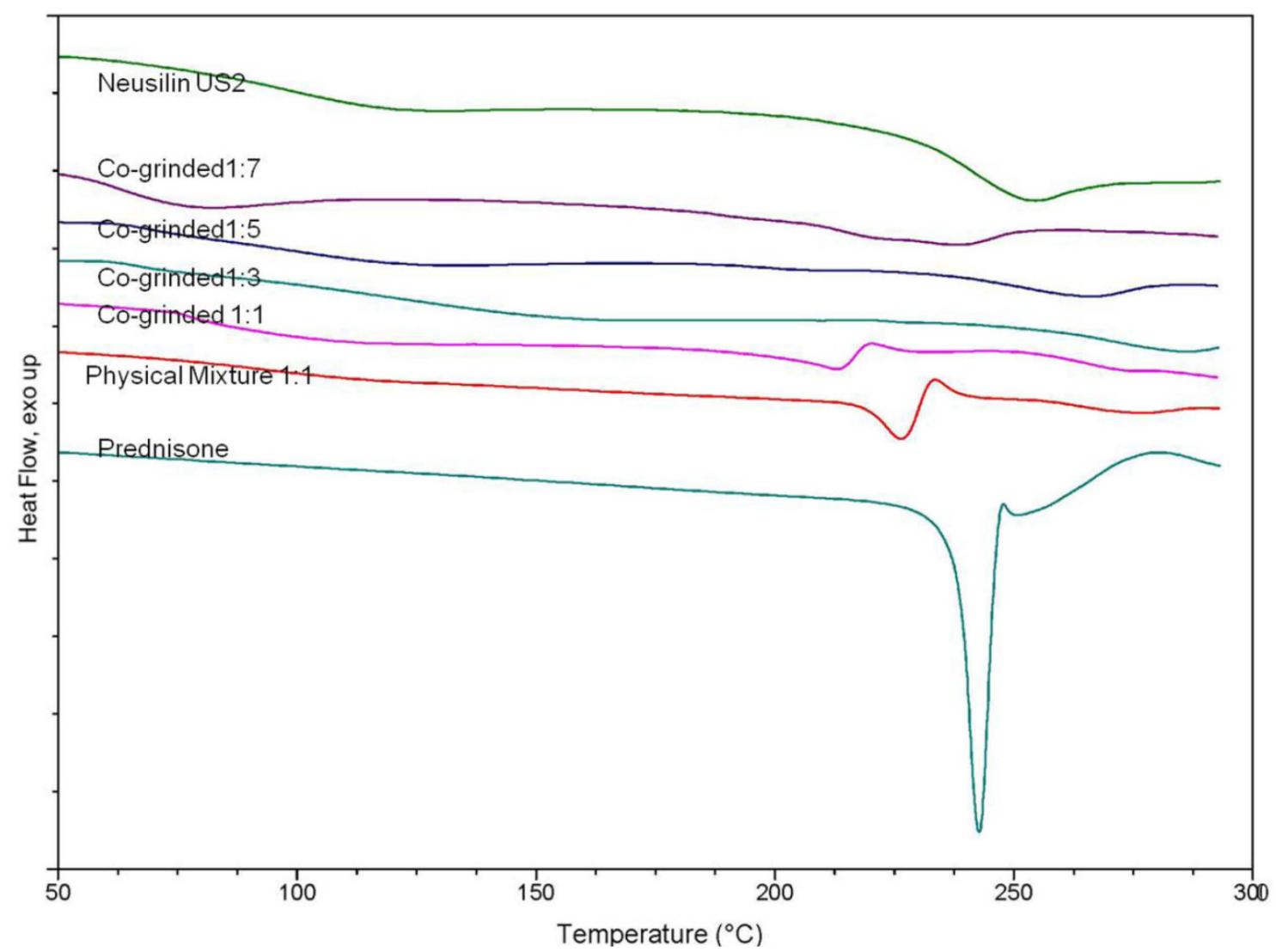

Figure 4-1. DSC patterns of various powder samples. 
Therefore, we reach a conclusion that amorphization of Prednisone was related to the amount of Neusilin. A possible explanation for this phenomenon suggests that as the total surface area of Neusilin ${ }^{\circledR}$ US2 increases, disrupted Prednisone slices would adhere and settle onto the surface of Neusilin ${ }^{\circledR}$ US2 granules, thus inhibiting the reversion back to the ordered crystalline structure. ${ }^{91}$

\subsubsection{X-ray powder diffraction}

Solids in crystalline state have intensive constructive reflections; however, those in amorphous state do not reflect X-ray beam since they do not have long-range molecular arrangement. The X-ray powder diffraction of conventional Prednisone and Prednisone/Neusilin complexes at different ratios are shown in Figure 4-2. The X-ray diffraction of Neusilin shows the absence of any peaks which exhibits the amorphous nature of Neusilin. Prednisone reveals multiple diffraction peaks including two predominantly split peaks at $14^{\circ}$ and one peak at $18^{\circ}$ as well as other minor peaks (percent crystallinity: 86.9). Both the 1:1 physical mixture and co-ground complexes indicate similar diffraction characteristics similar to conventional Prednisone. Moreover, and as expected, the intensity of X-ray diffraction of 1:1 co-ground complex was slightly smaller than that of 1:1 physical mixture. As the amount of Neusilin increased, all intensities of characteristic peaks decreased remarkably. When the ratio of Prednisone to Neusilin increased to $1: 7$, the two predominant split peaks at $14^{\circ}$ and the one at $18^{\circ}$ were extremely low in intensity and all other minor peaks completely disappeared (percent crystallinity: 25.1). Therefore, the X-ray diffraction suggested that most Prednisone in coground 1:7 complex was in amorphous state. We are led to the conclusion that the increase in the amount of Neusilin reduced the crystallinity of Prednisone which was consistent with previous DSC results.

\subsubsection{Solubility measurements}

According to previous literature, the solubility and dissolution rate enhancement of drug in amorphous state is caused by the formation of disordered structure of molecules which has more free energy as compared to the molecules in ordered crystalline structure. ${ }^{97}$ The solubility of conventional Prednisone and co-ground 1:7 Prednisone/Neusilin complex were evaluated as a function of time in Figure 4-3. Prednisone exists in the crystalline state with highly hydrophobic properties. Figure 4-3 showed that crystalline Prednisone reached an equilibrium solubility of $0.117 \mathrm{mg} / \mathrm{mL}$ within 3 hours then held a plateau for up to 72 hours. As for co-ground 1:7

Prednisone/Neusilin complex, Prednisone reached a maximal solubility at $0.28 \mathrm{mg} / \mathrm{mL}$ within 10 hours then plateaued for 72 hours. It was obvious that Prednisone/Neusilin complex displayed about two folds increase in solubility. Therefore, an amorphous Prednisone in the presence of Neusllin, could form a saturated solution and maintain solubility for at least 72 hours under this experimental condition. Additionally, solubility reduction was not observed in this experiment, which indicated that Neusilin granule may be an inhibitor for reducing the recrystallization of Prednisone in saturated solutions. 


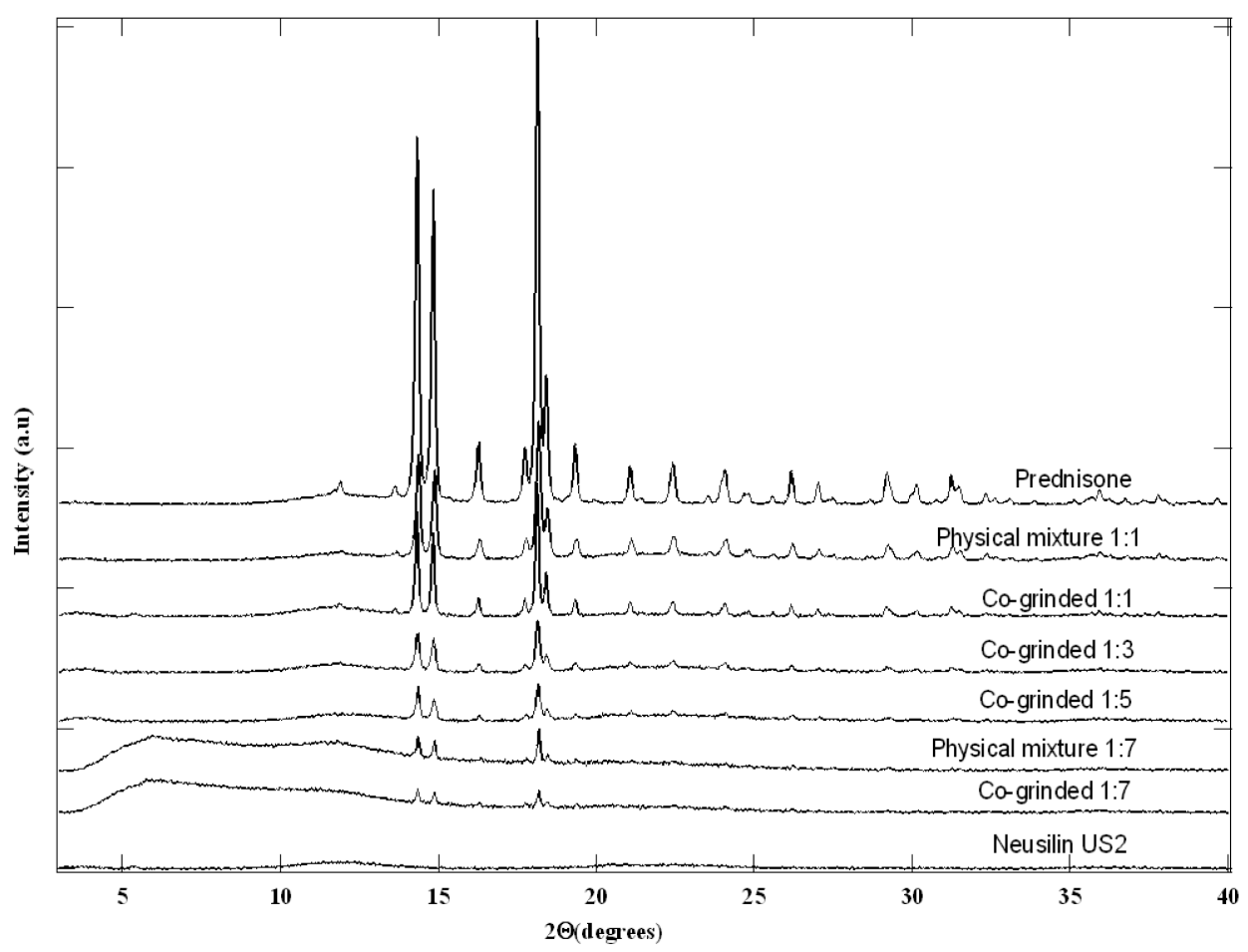

Figure 4-2. X-ray powder diffraction patterns of various Prednisone powder samples. 


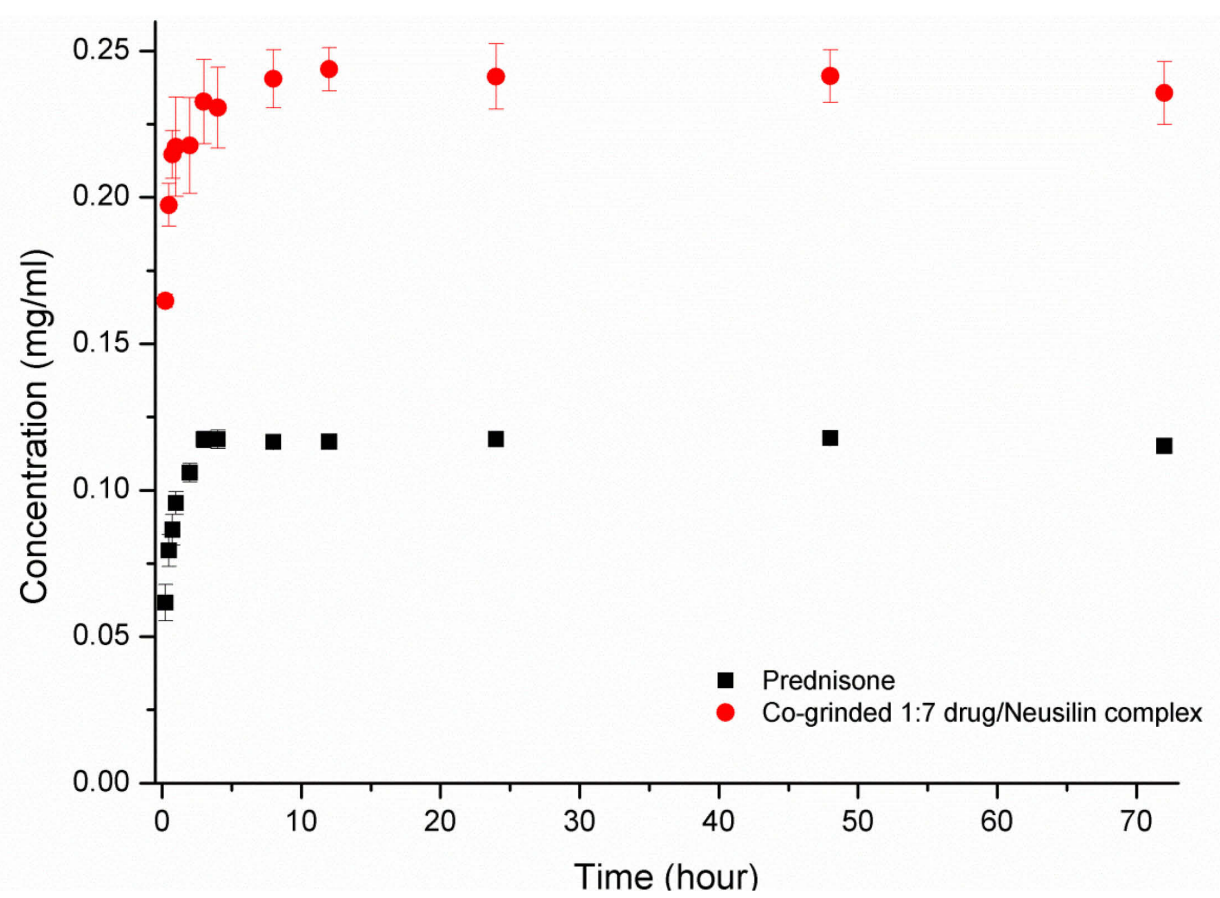

Figure 4-3. Kinetic solubility of Prednisone powder samples. 


\subsubsection{Surface area and particle size measurements}

It has been reported that the silanol groups which are situated on the surface of Neusilin granules act as potential hydrogen bond acceptors. ${ }^{91,92}$ Therefore, it is likely to form hydrogen bonds between Prednisone and silanol groups. Based on this assumption, amorphous Prednisone was expected to adsorb onto the surface of Neusilin ${ }^{\circledR}$ US2.

According to the literature, by combining the results of surface area and particle size analysis, the modality of the drug as either adsorbed to Neusilin surface or kept free in the crystal form in addition to the state of Neusilin granule could be evaluated. ${ }^{90}$ Results of particle size and surface area measurements were listed in Table 4-1. The manufacturer of Neusilin ${ }^{\circledR}$ US2 reported that the mean particle size ranged from 60 to $120 \mu \mathrm{m}$ and the specific surface area of $300 \mathrm{~m}^{2} / \mathrm{g}$. This was consistent with the experimental values that we obtained milled Neusilin ${ }^{\circledR}$ US2 (mean particle size $88.62 \mu \mathrm{m}$; surface area $329.60 \mathrm{~m}^{2} / \mathrm{g}$ ). Hence, Neusilin ${ }^{\circledR}$ US2 granule was intact without fracture or breakage during the milling process under this shaking frequency. The high surface area of Neusilin is related to its porous nature and the exposed surface.

Prednisone crystals had smaller mean particle size of $6.36 \mu \mathrm{m}$ and surface area of $3.31 \mathrm{~m}^{2} / \mathrm{g}$. To further clarify Prednisone adsorption to Neusilin, we need to pinpoint that Prednisone crystals were disrupted during ball milling and slices of Prednisone in the amorphous form were adsorbed to Neusilin. Thus, the particle size distribution of co-ground Prednisone/Neusilin complex was expected to be similar to that of Neusilin granule alone. On the other hand, the surface area of the complex was lower than that of Neusilin granules because Prednisone slices covered and/or occupied the surfaces of Neusilin. Mean particle size values of co-ground 1:7 Prednisone/Neusilin complex was $87.74 \mu \mathrm{m}$ and total surface area was $271.79 \mathrm{~m}^{2} / \mathrm{g}$. Therefore, this explains the particle size distribution and the lower surface area of Prednisone as compared to those of Neusilin. It was noted that as the content of Neusilin in the complex decreased, particle size distribution $\mathrm{D}_{10}, \mathrm{D}_{50}, \mathrm{D}_{90}$, and $\mathrm{M}_{\mathrm{v}}$ shifted to smaller values. This in part is due to the existence of drug crystals in 1:1 complex (mean particle size: $66.67 \mu \mathrm{m}$ ) and 1:3 complex (mean particle size: $75.95 \mu \mathrm{m}$ ). Moreover, the decrease of Neusilin content in the complex resulted in a decrease in surface area; e.g. 1:1 complex (surface area: $157.15 \mathrm{~m}^{2} / \mathrm{g}$ ); 1:3 complex (surface area: $229.04 \mathrm{~m}^{2} / \mathrm{g}$ ). These surface area values could be reasoned by (a) drug crystals that remained in the complex after co-grinding had relatively smaller surface area; and (b) porous surface on the Neusilin were covered by amorphous drug so that surface area was further decreased.

\subsubsection{Morphology}

The morphologies of Prednisone and Neusilin were directly visualized by Scanning Electron Microscope (SEM). This experiment enabled us to observe the adsorption of Prednisone to Neusilin surfaces. As seen in Figure 4-4, after cogrinding (ball milling), Neusilin ${ }^{\circledR}$ US2 granules were kept intact. It could be seen that Neusilin granules had a spherical shape and wide particle size distribution as was also verified by particle size measurement using laser diffraction. In addition, the surfaces of granules were not smooth, rather porous. 
Table 4-1. Surface area and particle size analysis of various powder samples.

\begin{tabular}{cccccc}
\hline Sample & $\mathbf{D}_{\mathbf{1 0}}(\boldsymbol{\mu m})$ & $\mathbf{D}_{\mathbf{5 0}}(\boldsymbol{\mu m})$ & $\mathbf{D}_{\mathbf{9 0}}(\boldsymbol{\mu m})$ & $\mathbf{M}_{\mathbf{v}}(\boldsymbol{\mu m})$ & Surface $\mathbf{A r e a ~}\left(\mathbf{m}^{\mathbf{2}} / \mathbf{g}\right)$ \\
\hline Prednisone & 1.44 & 4.83 & 12.12 & 6.36 & 3.31 \\
Co-grinded 1:1 D/N & 5.71 & 54.14 & 144.05 & 66.67 & 157.15 \\
Co-grinded 1:3 D/N & 11.44 & 78.48 & 144.06 & 75.95 & 229.04 \\
Co-grinded 1:5 D/N & 16.16 & 88.81 & 158.59 & 83.29 & 252.07 \\
Co-grinded 1:7 D/N & 33.24 & 90.85 & 160.58 & 87.74 & 271.79 \\
Milled Neusilin $\AA$ & 34.16 & 90.89 & 161.63 & 88.62 & 329.60 \\
\hline
\end{tabular}

D/N: Drug/Neusilin Complex.
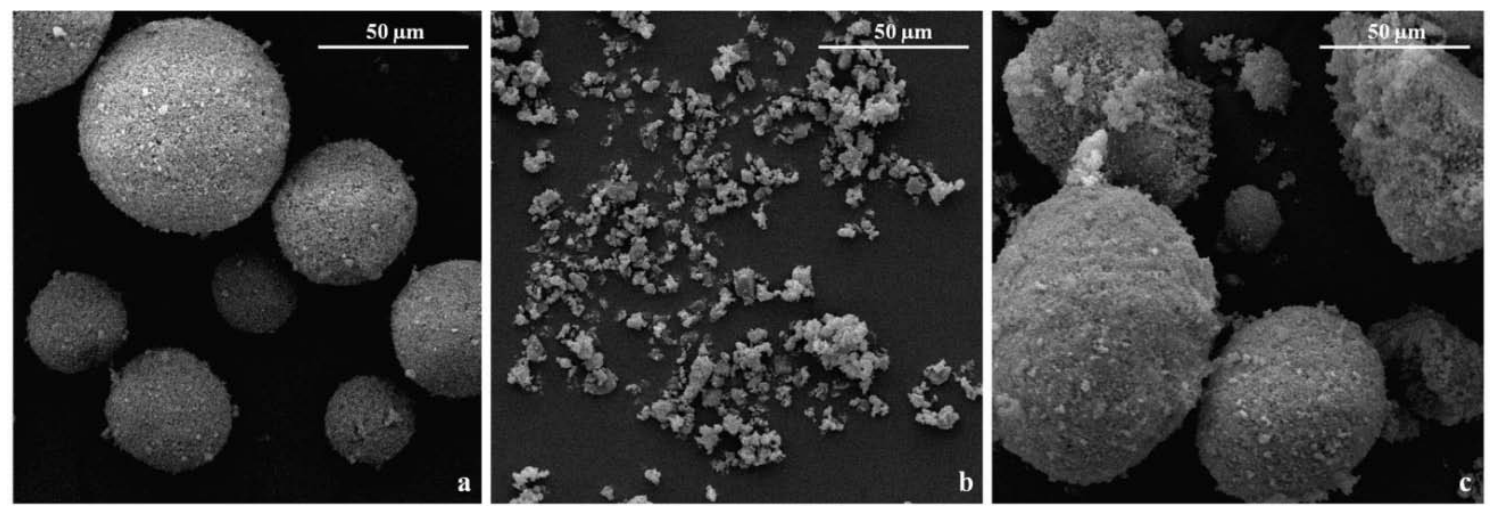

Figure 4-4. SEM micrographs of Prednisone powder samples.

(a) Neusilin; (b) Prednisone; (c) Co-grinded 1:7 drug/Neusilin complex. 
It was noted the crystals had a smaller size as compared to Neusilin granules. The particle size of Prednisone as obtained by SEM was less than $10 \mu \mathrm{m}$. This is consistent with the previous particle size measurement. The morphology of co-ground 1:7 complex is shown in Figure 4-4. We noticed that most Prednisone particles were adsorbed onto the surface of Neusilin and only some particles were left free. This observation verified our assumption that Neusilin functioned as an adsorbent that enabled the amorphization of Prednisone. It was also observed that the porous surface of Neusilin was partially covered by Prednisone particles which were responsible for surface area reduction of Neusilin granule. Finally we need to mention that Neusilin maintained its original spherical shape and size after grinding.

\subsubsection{Characterization of core mini-tablets}

The three mini-tablet formulations which were prepared are shown in Table 4-2. The unit dose of Prednisone in each tablet was about $0.5 \mathrm{mg}$ which was therapeutically appropriate for pediatric indications. Tablet thickness, total weight, and drug content were consistent among the three formulations which suggest that single compression maintained constant mini-tablet characteristics during the compression process. In addition, results of tensile strength $(4.01 \pm 0.31 \mathrm{MPa})$ and friability tests $(0.21 \%)$ indicated that mini-tablets of Formulation 1 containing co-ground 1:7 Prednisone/Neusilin complex had acceptable mechanical properties.

\subsubsection{Powder and core mini-tablet dissolution profiles}

Dissolution profiles of co-ground Prednisone/Neusilin complexes at different ratios of 1:1, 1:3, 1:5, 1:7 and conventional (crystalline) Prednisone are shown in Figure 4-5. The dissolution profile of crystalline Prednisone (control) indicated that up to $30 \%$ of the drug had dissolved within 20 minutes and around $70 \%$ dissolved within 90 minutes. It is worth mentioning from these results that the amounts dissolved were related to the weight ratio of Prednisone to Neusilin.

The observed differences in dissolution rates were related to the co-existence of amorphous and crystalline forms of Prednisone. At the low ratio of Prednisone in the complexes (i.e. a ratio of 1:7), more amorphous form resulted in faster dissolution rate. This was consistent with the observations of other researchers who investigated dissolution rate enhancement caused by different ratios of drug to excipient. ${ }^{98,99}$ As the Neusilin ratio decreased, more Prednisone is present in the crystalline state and less amorphous form is adsorbed onto the surface of Neusilin granules which leads to slower dissolution rate. Moreover, it is important to mention that incomplete release of amorphous form may also be related to the formation of gel-like mass, which was discussed by Law and co-workers. ${ }^{100}$ In order to prepare mini-tablets, and since powder complex ratio at 1:7 exhibited the fastest dissolution profile as shown in Figure 4-5, a coground Prednisone to Neusilin complex at 1:7 was selected for mini-tablet (Formulation 1). In addition, physical mixture at the same ratio and crystalline Prednisone mini-tablets 
Table 4-2. Characteristics of mini-tablets.

\begin{tabular}{cccccc}
\hline Formulation & $\begin{array}{c}\text { Thickness } \\
(\mathbf{m m})\end{array}$ & $\begin{array}{c}\text { Weight } \\
\mathbf{( m g )}\end{array}$ & $\begin{array}{c}\text { Unit Dose } \\
\text { Strength } \mathbf{( m g )}\end{array}$ & $\begin{array}{c}\text { Tensile Strength } \\
\mathbf{( M P a )}\end{array}$ & $\begin{array}{c}\text { Friability } \\
\mathbf{( \% )}\end{array}$ \\
\hline 1 & $1.93 \pm 0.03$ & $5.23 \pm 0.14$ & $0.55 \pm 0.01$ & $4.01 \pm 0.31$ & 0.21 \\
2 & $1.92 \pm 0.01$ & $5.23 \pm 0.19$ & $0.54 \pm 0.01$ & NA & NA \\
3 & $1.84 \pm 0.01$ & $5.40 \pm 0.25$ & $0.56 \pm 0.01$ & NA & NA \\
\hline
\end{tabular}




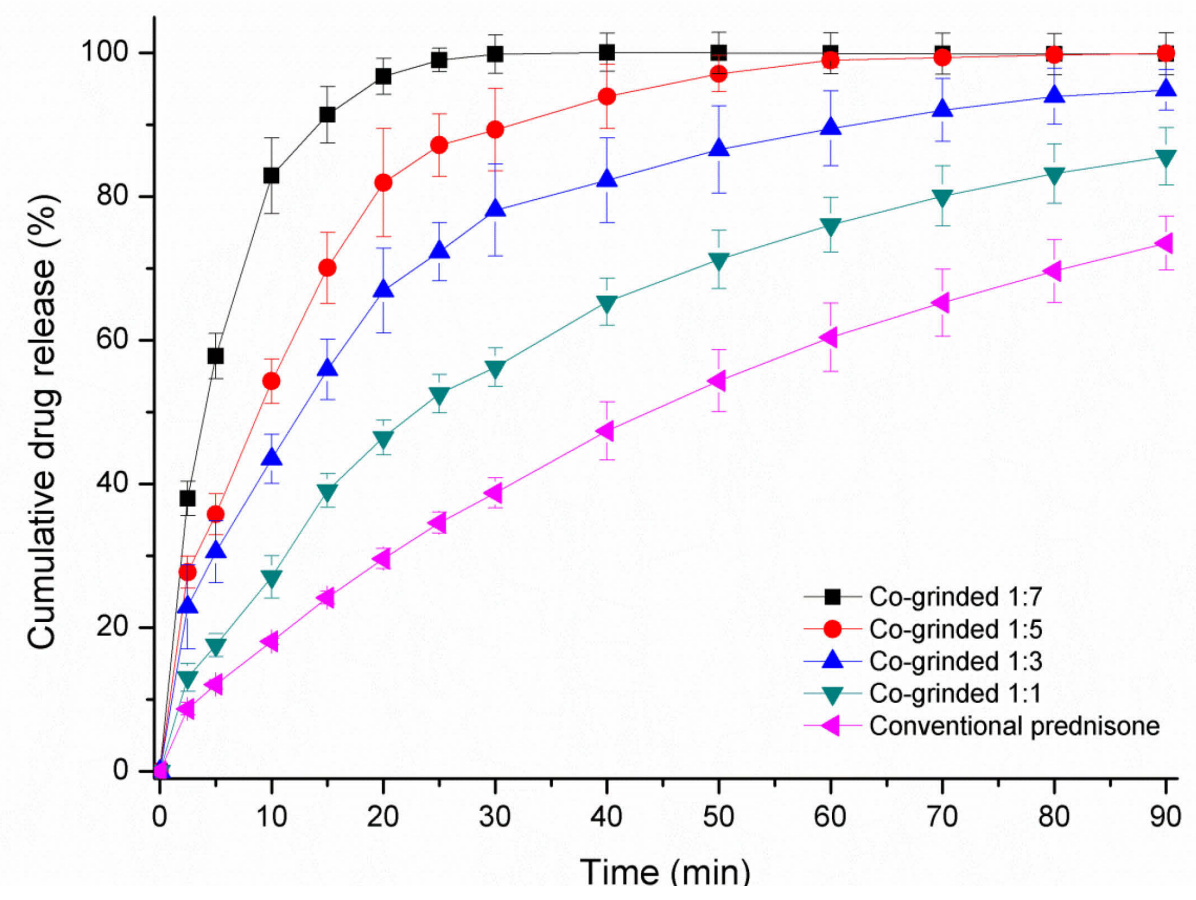

Figure 4-5. Dissolution profiles of Prednisone powder samples. 
were also utilized as controls (Formulations 2 and 3 ).

The dissolution profiles of the three mini-tablets (Formulations 1, 2 and 3) which were described are shown in Figure 4-6. The mini-tablets dissolution profiles in Figure 4-6 show that 1:7 co-ground Prednisone/Neusilin tablets exhibited the fastest dissolution profile ( $87 \%$ in 20 minutes) followed by $1: 7$ physical mixture $(68 \%$ in 20 minutes) then the mini tablet with crystalline Prednisone (60\% in 20 minutes). Previous reports indicated that the disintegration time can positively influence the dissolution rate of the drug from tablets. ${ }^{101}$ Therefore, mini-tablets are expected to disintegrate within a short period of time in order to release Prednisone rapidly in gastric fluids. The presence of Neusilin in the formulation improves the hardness and may cause a delay to the disintegration process. In order to obtain a reasonable disintegration time, croscarmellose sodium was added as a super-disintegrant. The dissolution profiles presented in Figure 4-6 indicates that disintegration of mini-tablets was acceptable and did not inhibit the dissolution rate of Prednisone. It is important to mention that crystalline form of Prednisone in Formulations 2 and 3 dissolved much faster as compared to Prednisone powders particularly during the initial stage. A possible explanation is linked to the hydrophilic properties of the co-blended excipients which include silicified microcrystalline cellulose and/or Neusilin. Both can enhance the wettability of Prednisone powder. We can conclude from Figure 4-6 that Formulation 1 showed an enhanced dissolution rate of Prednisone; therefore it is reasonable to say that Prednisone remained in amorphous state during mini-tablet preparation and dissolution processes.

\subsubsection{Coated mini-tablets dissolution profiles}

Film coating of tablets is a widespread approach to inhibit contact between a drug with unpleasant-taste and sensors of taste buds. It was reported that suppression of drug release for only few minutes at the initial stage could reduce unpleasant sensations associated with bitter taste of drugs. ${ }^{102}$ Eudragit ${ }^{\circledR}$ EPO, a pH-dependent copolymer, is insoluble at $\mathrm{pH}$ above 5 but becomes soluble at $\mathrm{pH}$ below 5 . Therefore, this polymer has been selected as an appropriate material for taste masking purpose. ${ }^{29,103}$ Figure 4-7 shows release behavior of Prednisone within coated mini-tablet in both simulated saliva solution $(\mathrm{pH}=6.8)$ and in simulated gastric fluid solution $(\mathrm{pH}=1.2)$. It was observed that when coated mini-tablets were placed in simulated saliva, the shape of the tablets was unchanged for approximately 70 to 90 seconds then afterwards gel-like flocculants were formed. Hypothetically, this may be due to the incomplete disintegration of coated minitablets after water penetration across Eudragit ${ }^{\circledR}$ EPO coating layer. The dissolution profile of coated mini-tablet in simulated saliva confirmed the assumption that Eudragit $\mathbb{R}$ EPO coating layer suppressed Prednisone release significantly ( $2 \%$ within 2.5 minutes). However, in simulated gastric fluid, Eudragit ${ }^{\circledR}$ EPO dissolved rapidly and Prednisone has dissolved with no barrier.

It is well known that exposure to moisture accelerates the re-crystallization

process of amorphous drugs and reduces the drug dissolution rate. ${ }^{104}$ Eudragit ${ }^{\circledR}$ EPO coating has been noted to protect against moisture penetration. ${ }^{29}$ It is expected in this 


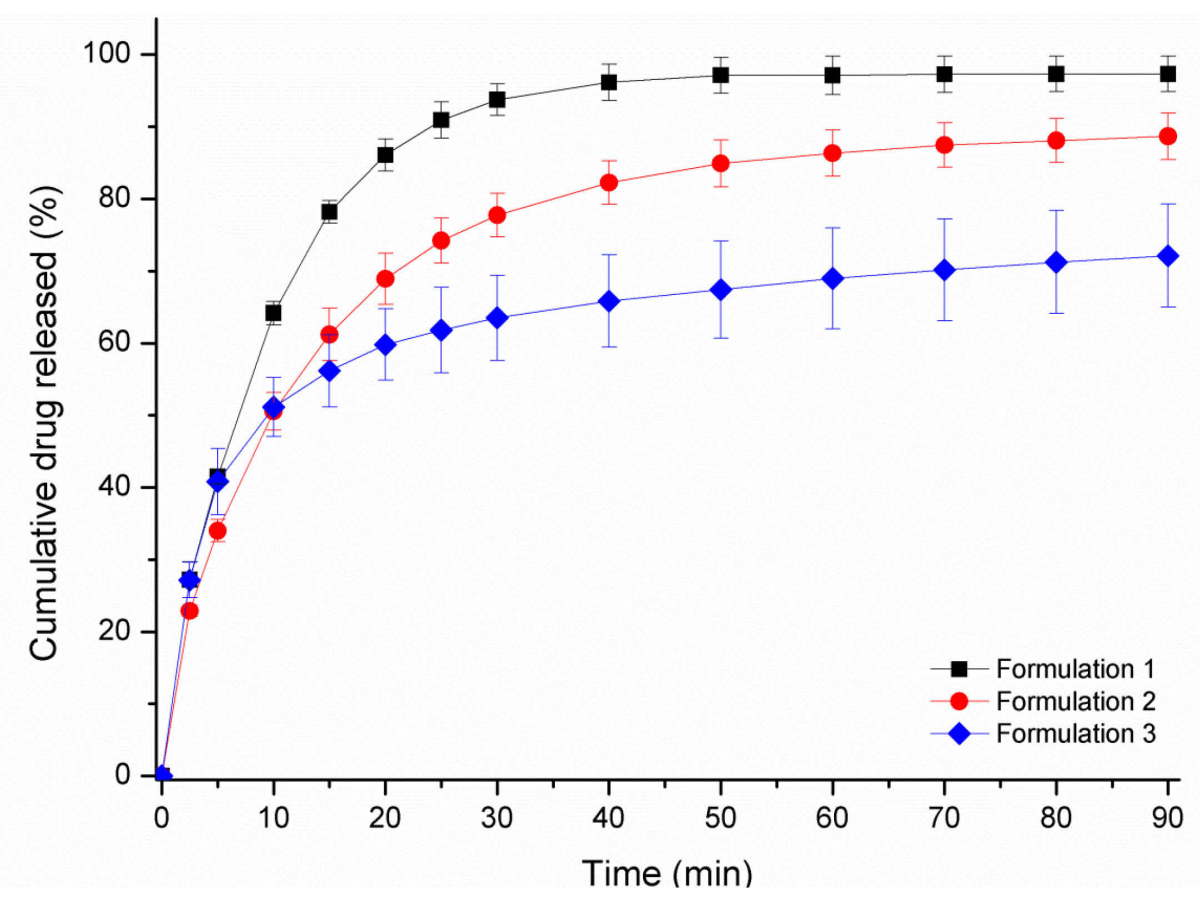

Figure 4-6. Dissolution profiles of core mini-tablets. 


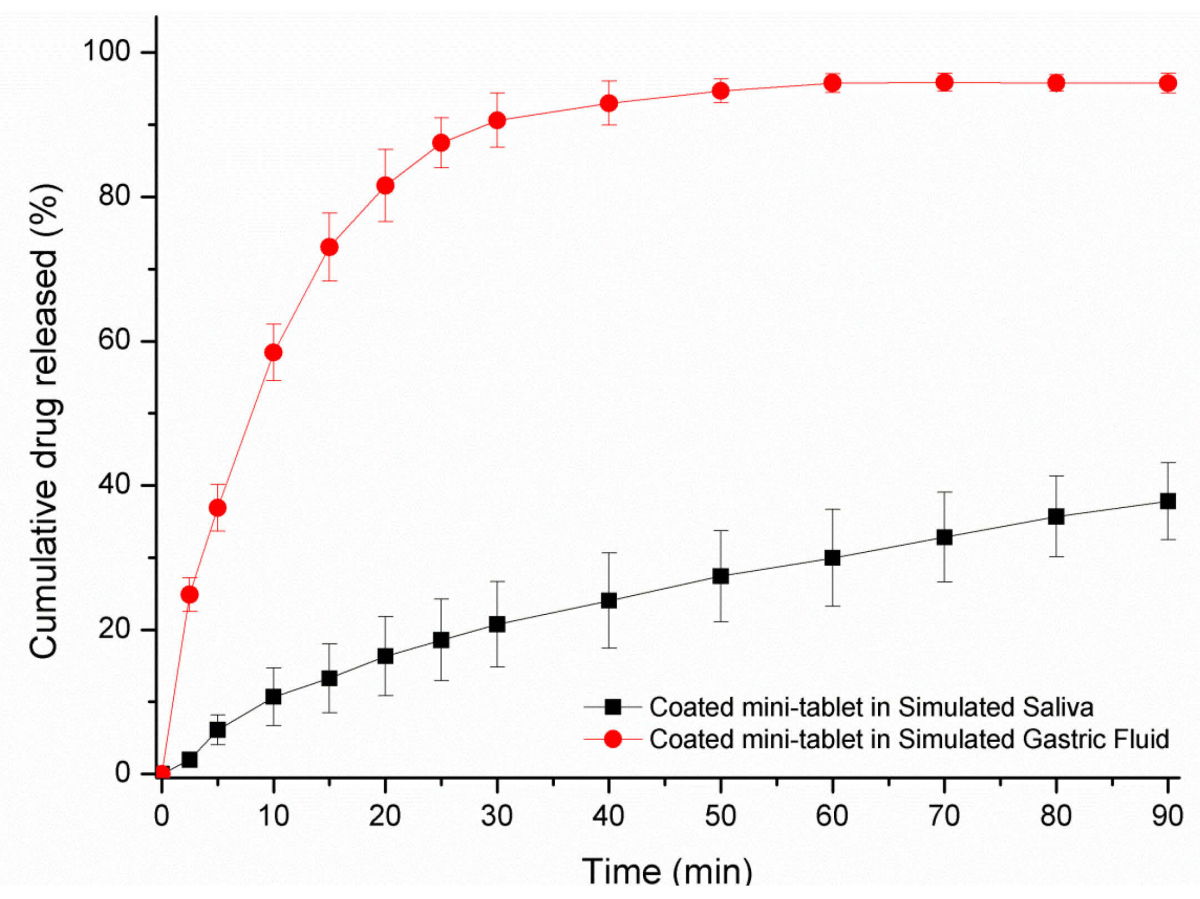

Figure 4-7. Dissolution profiles of coated mini-tablets. 
study that it would decrease the moisture uptake of amorphous Prednisone, thereby decreasing the re-crystallization process. Drug release behavior of Formulations 1 and 4 (after 1 month of physical stability evaluation) in simulated gastric fluid were plotted and shown in Figure 4-8. The similarity factor value $\left(f_{2}\right)$ was calculated and a comparison was made between the release profile of fresh coated mini-tablets (Formulation 4), and coated mini-tablets (Formulation 4 ) after one month on physical stability (the $\mathrm{f}_{2}$ value was 66.5). Also, a comparison was made between release profiles of fresh core minitablets (Formulation 1) and core mini-tablets (Formulation 1) after one month on physical stability, (the $\mathrm{f}_{2}$ value was 46.6). In addition, a comparison was made between release profiles of coated mini-tablets (Formulation 4) after one month on physical stability and core mini-tablets (Formulation 1) after one month of physical stability (the $\mathrm{f}_{2}$ value was 70.7) respectively.

These values indicate that although there is no significant difference between drug release profiles for coated and uncoated mini-tablets after 1-month of stability testing, Eudragit ${ }^{\circledR}$ EPO coating had some influence on maintaining (moisture decreases dissolution rate and therefore coating helps to maintain dissolution rate) drug dissolution rate. A possible reason is that Eudragit ${ }^{\circledR}$ EPO coating somewhat decreased the exposure of drug to moisture, which can minimize the drug re-crystallization; however, it was concluded that re-crystallization of amorphous drug could not totally be inhibited by the Eudragit ${ }^{\circledR}$ EPO coating during this limited time frame of 4 weeks. We like to show a visual comparison in size between Formulations 1 and Formulation 4 (see Figure 4-9).

\subsection{Conclusion}

This study demonstrated that co-ground Prednisone-Neusilin complex enhanced drug dissolution rate due to a decrease in Prednisone crystallinity. Co-ground PrednisoneNeusilin (1:7) complex yielded reliable and reproducible preparation processes for minitablets. Eudragit ${ }^{\circledR}$ EPO coating (taste masking) suppressed the drug release from minitablets in simulated saliva $(\mathrm{pH}=6.8)$ while maintained immediate release properties in simulated gastric media $(\mathrm{pH}=1.2)$. Thus, due to its small size, coated and uncoated minitablets of co-ground Prednisone-Neusilin can potentially be appropriate for pediatric use. 


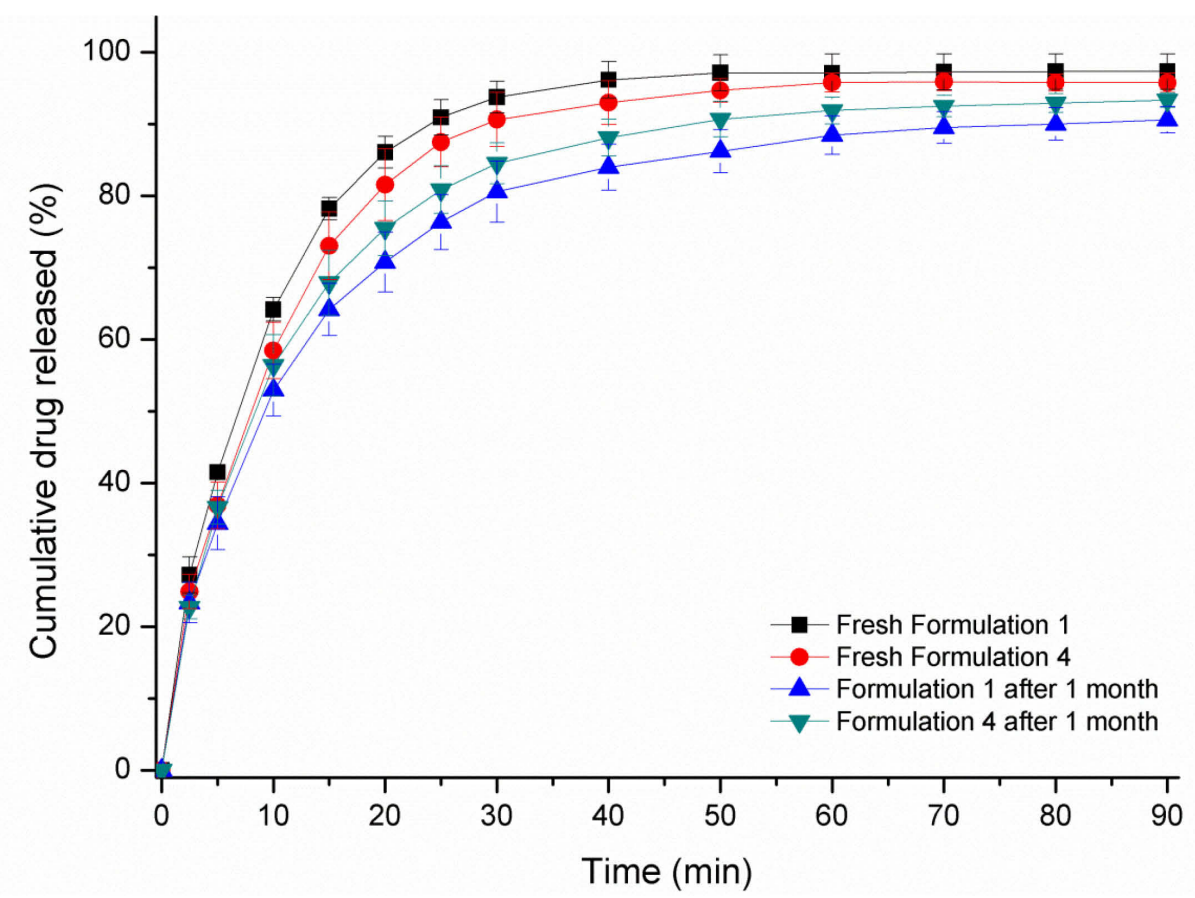

Figure 4-8. Dissolution profiles of mini-tablets after 1-month stability test. 


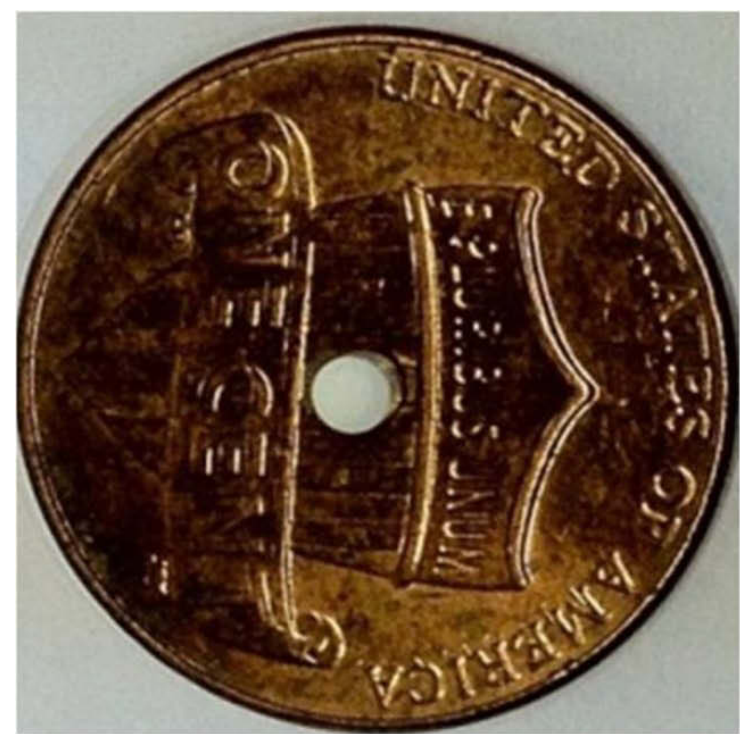

Formulation 1

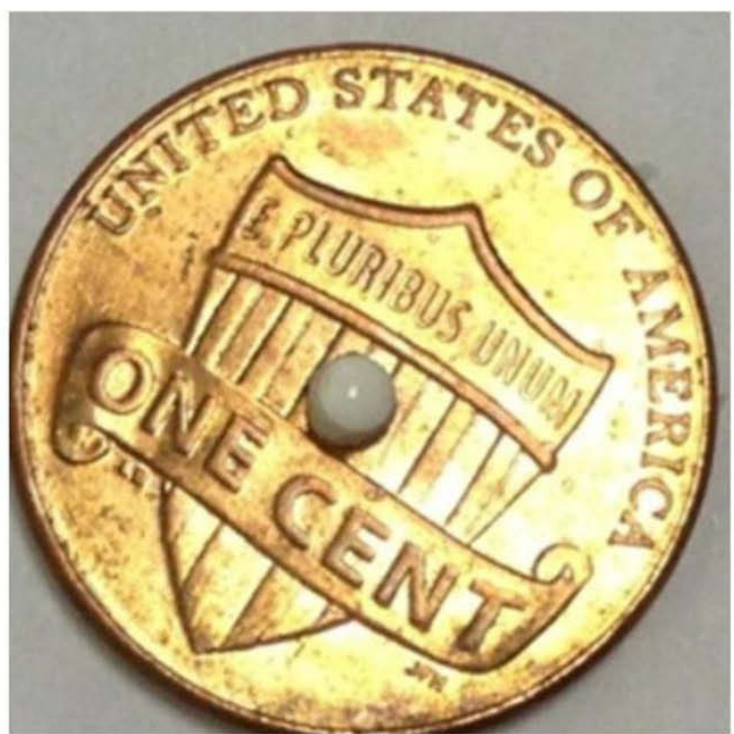

Formulation 4

Figure 4-9. Photos of Formulation 1 and Formulation 4. 


\section{CHAPTER 5. DEVELOPMENT OF W/O MICROEMULSION FOR TRANSDERMAL DELIVERY OF IODIDE IONS *}

\subsection{Introduction}

Iodide is vital for the biosynthesis of thyroid hormones triiodothyronine (T3) and thyroxine (T4) in the thyroid gland. The deficiency of T3 and T4 leads to thyroid tissue enlargement and other metabolic and physiological problems. It has been illustrated by previous studies that iodide is massively accumulated in the thyroid gland regardless of the dosing route. ${ }^{105,106}$ This accumulation is regulated by sodium-iodide symporter which transports iodide from blood into thyroid epithelial cells. ${ }^{107,108}$ Typically, small amount of iodide is used as a daily nutritional supplement to prevent iodine deficiency, ${ }^{109}$ whereas much larger doses are used to avoid thyroid uptake of radioactive iodide following nuclear fission accidents. ${ }^{110,111}$ Iodide is present in the typical diet, primarily through the use of iodized table salt. Oral iodide can also be supplemented as potassium iodide (available in both tablet and solution dosage forms) as well as with iodine strong solution (Lugol's solution). However, oral administration may not be ideal in certain populations at greatest risk for iodine deficiency, such as infants or patients who suffered surgical removal of GI tract. For this reason, transdermal drug delivery system may be an appropriate alternative to oral delivery, particularly when oral absorption is compromised in disease states characterized by malabsorption (e.g., short bowel syndrome). In contrast to other drug delivery systems, transdermal administration offers advantages including convenient, non-invasive, and continuous dosing and the avoidance of first-pass metabolism. ${ }^{112}$

It is well understood that low molecular weight hydrophilic compounds including ionized compounds can permeate through skin by appendage shunt pathways such as hair follicles and sweat glands. ${ }^{113,114,115}$ However, the total amount of drug which can be diffused via this route is limited because of its small surface area compared to the total skin. In addition, it has been proposed that small ions can diffuse through lipid bilayer of the stratum corneum by the "aqueous" or the "pore" pathway model. ${ }^{116,117,118}$ In this model, pores are formed as a result of defects or imperfections in the interior structure of lipid bilayer which leads ions to travel through more rigid tortuous routes. ${ }^{119,120}$ Depending on this model, ion diffusion through stratum corneum can be improved by altering the porosity of lipid bilayer. Various types of penetration enhancers, such as water, surfactants, fatty acids, and azones, can effectively influence the porosity of the stratum corneum and further lower its resistance to chemicals. ${ }^{23}$ On the other hand, ion penetration through the skin is also influenced by ion's permeability. A recent report demonstrated that anions have a faster diffusion rate than cations due to the presence of negatively charged phospholipid groups in the stratum corneum. ${ }^{121}$ These findings

* : Adapted with permission. Lou H, Ni Q, Crill C, Helms R, Almoazen H. Development of W/O microemulsion for transdermal delivery of iodide ions. AAPS PharmSciTech 2013;14:168-76. 
support our intent to deliver iodide ions through microemulsions. Microemulsion is a multicomponent system composed of water, oil, surfactant, and cosurfactant. ${ }^{21}$ It has been intensively utilized as a transdermal delivery system with several advantages including low cost and simple preparation, long term product stability, and main ingredients acting as solubilization and permeation enhancers. ${ }^{20,22,122}$ Microemulsion can potentially change the internal structure of the lipid bilayer in the stratum corneum and enhance compound penetration. Our aim is to develop an iodide transdermal delivery system which has potential therapeutic uses when oral administration is not appropriate. In this study, a $\mathrm{W} / \mathrm{O}$ microemulsion system with potassium iodide (KI) was developed. Several physicochemical characterizations were conducted to evaluate the system (e.g. pH, droplet size, conductivity, viscosity, and stability). Franz diffusion cells were also utilized to evaluate the penetration of the iodide ions through human skin.

\subsection{Experimental Procedures}

\subsubsection{Materials}

Potassium iodide (KI), Span 20 and Pyrene were purchased from Sigma Aldrich (St. Louis, MO, USA). Anhydrous ethanol was purchased from Fisher Scientific (Thermo Fisher Scientific Inc, Pittsburgh, PA, USA). Capryol 90® was obtained from Gattefosse (Lyon, France). De-ionized water was used in this study.

\subsubsection{Construction of pseudo-ternary phase diagrams}

Pseudo-ternary phase diagrams were constructed to evaluate the miscibility of the basic components in the system at $25^{\circ} \mathrm{C}$. A series of different ratios $\left(\mathrm{K}_{\mathrm{m}}\right)$ of surfactant (Span 20) to cosurfactant (ethanol) were prepared at 4:1, 1:1, 1:4, and 1:9, and then followed by the addition of oil (Capryol 90®) at different weight ratios of oil to mixture of surfactant and cosurfactant of 10:0, 9:1, 8:2, 7:3, 6:4, 5:5, 4:6, 3:7, 2:8 and 1:9 respectively. Water was titrated drop by drop to the three-component mixture (under constant magnetic stirring) until a transition point where transformation from transparent (optical monophase) to turbid (optical diphase) was reached. A boundary line connecting all transition points was drawn, and the monophasic area $\mathrm{A}_{\mathrm{T}}$ beneath this boundary line was calculated using Origin 8 software (OriginLab Corporation, Northhampton, MA), which is shown in Figure 5-1. A $A_{T}$ was used to evaluate water solubilization capacity into the oil. The following solubilization capacities $A_{T}$ were calculated at different surfactant:cosurfactant ratio $\mathrm{K}_{\mathrm{m}}\left(\mathrm{K}_{\mathrm{m}}: 4: 1, \mathrm{~A}_{\mathrm{T}}: 16.6, \mathrm{~K}_{\mathrm{m}}: 1: 1, \mathrm{~A}_{\mathrm{T}}: 28.5, \mathrm{~K}_{\mathrm{m}}: 1: 4, \mathrm{~A}_{\mathrm{T}}: 35.7\right.$, $\left.\mathrm{K}_{\mathrm{m}}: 1: 9, \mathrm{~A}_{\mathrm{T}}: 37.4\right)$. The pseudo-ternary phase diagram at a constant surfactant/cosurfactant ratio $\mathrm{K}_{\mathrm{m}}$ of 1:1 was chosen for further development because there is sufficient area in pseudo-ternay phase diagram which could form micromeulsion at this ratio while cosurfactant (ethanol) was kept relatively low in the formulation. A dilution line (L20) was plotted linking $100 \%$ water to a mixture of oil and S/COS of $20 \%$ and $80 \%$ in

\section{Figure 5-2.}



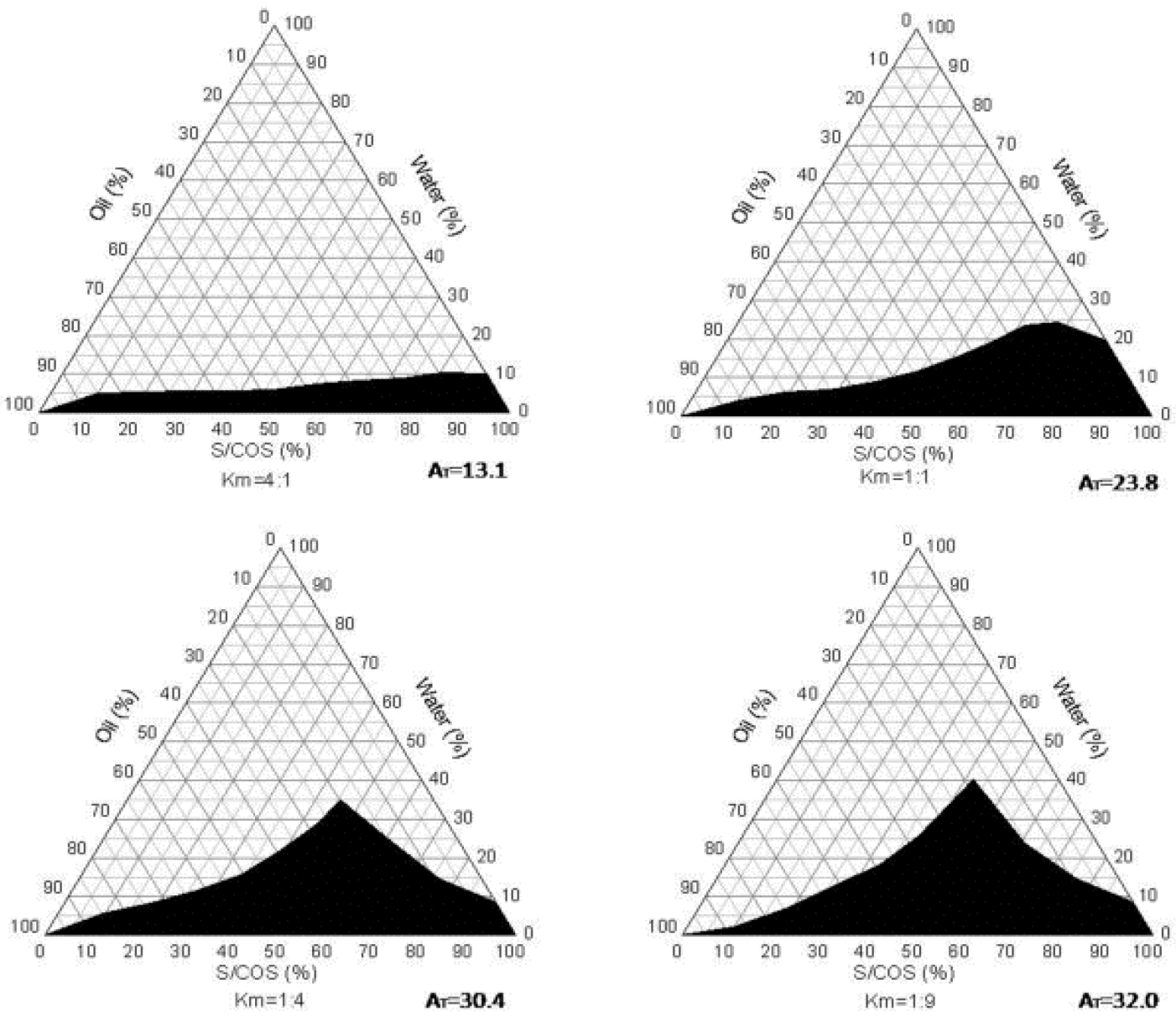

Figure 5-1. Pseudo-ternary phase diagrams of mixtures composed of oil (Capryol $90 \AA)$, water, surfactant (Span 20), and cosurfactant (ethanol) at various $\mathrm{S} / \mathrm{COS}$ ratios (Km).

Shaded area represents the domain where the mixture system is monophasic. 


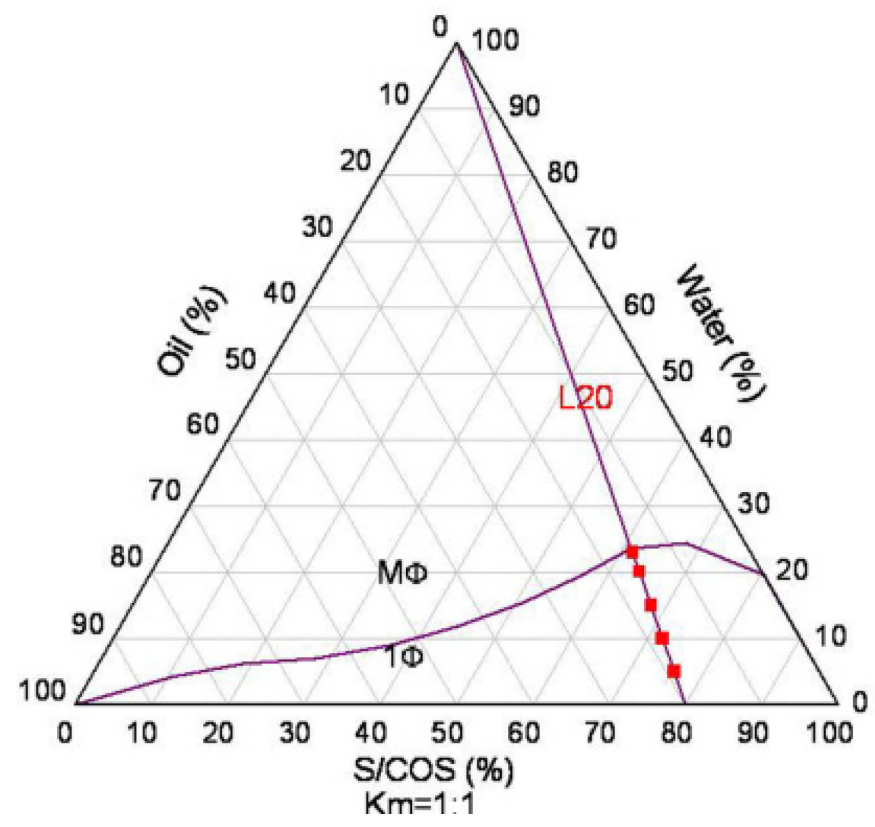

Figure 5-2. Formulation selection from pseudo-ternary phase.

L20 is a dilution line which connects all formulations with a fixed ratio (20/80) of oil to $\mathrm{S} / \mathrm{COS}$. Below the purple boundary line, the mixture exists as one phase, which is represented by $1 \varphi$. Otherwise, the mixture is of multiple phases, which is represented by $\mathrm{M} \varphi$. 
Data points on this line have a constant ratio of oil to S/COS of 1:4. ${ }^{123,124}$ The intersection between the dilution and the boundary lines was recognized as the formulation with maximum water solubilization capacity. Five selected formulations (Table 5-1) with different water contents from L20 were further tested. Blank formulations were first prepared by manually mixing Span 20, ethanol, Capryol $90 \AA$, and water. Then formulations were incorporated with potassium iodide (KI) at a constant concentration of $50 \mathrm{mg} / \mathrm{mL}$ by vortex mixing.

\subsubsection{Droplet size measurements}

The mean droplet size of selected formulations was determined by dynamic light scattering using Zetasizer Nano ZS (Malvern Instruments Inc, Westborough, MA, USA). Light was scattered at a fixed angle of $90^{\circ}$. Refractive index and viscosity values were inputted into the program to determine the mean droplet size accurately. All measurements were obtained at $25{ }^{\circ} \mathrm{C}$. Triplicate measurements were taken.

\subsection{4. $\mathrm{pH}$ measurements}

The $\mathrm{pH}$ values of selected formulations were acquired using Orion 520A $\mathrm{pH}$ meter (Thermo Fisher Scientific Inc, Pittsburgh, PA, USA). The $\mathrm{pH}$ probe was inserted into $20 \mathrm{~mL}$ of liquids and values were recorded when the reading stabilized. All measurements were done in triplicate.

\subsubsection{Viscosity measurements}

The kinematic viscosities of the selected formulations were determined using Cannon-Fenske routine viscometer (Cannon Instrument Company, State College, PA, USA) at ambient temperature. Kinematic viscosity was obtained by multiplying efflux time of sample flowing through the capillary tube of the viscometer by the viscometer constant. Thereafter, the dynamic viscosity was determined by multiplying the value of kinematic viscosity by the sample density. All measurements were performed in triplicate.

\subsubsection{Conductivity measurements}

Conductivity measurements were performed using conductivity meter FE30/FG3 (Mettler-Toledo Inc, Columbus, OH, USA) at $25^{\circ} \mathrm{C}$. Conductivity diagram was obtained through drop by drop water titration to the mixture of oil and surfactant/cosurfactant $(\mathrm{S} / \mathrm{COS})$ at a constant ratio of 1:4 in a beaker. The conductivity sensor was soaked in the liquid and the reading was recorded when the signal indicating the endpoint was achieved. The conductivity of the five selected formulations after the incorporation of KI was carried out using the same methodology. All measurements were carried out in triplicate. 
Table 5-1. Formulation composition, $\mathrm{pH}$ and $\mathrm{Z}$-average diameter at $25^{\circ} \mathrm{C}$.

\begin{tabular}{ccccc}
\hline Formulation & $\begin{array}{c}\text { Water/Span20/Ethanol/ } \\
\text { Capryol 90® } \\
\text { Composition (w/w } / \mathbf{w})\end{array}$ & KI & pH & $\begin{array}{c}\text { Z-average } \\
\text { Diameter (nm) }\end{array}$ \\
\hline $\mathrm{A}$ & $5 / 38 / 38 / 19$ & None & $5.20 \pm 0.01$ & Size under detection \\
& & & & limit \\
$\mathrm{B}$ & $10 / 36 / 36 / 18$ & None & $5.13 \pm 0.01$ & $1.48 \pm 0.03$ \\
$\mathrm{C}$ & $15 / 34 / 34 / 17$ & None & $5.00 \pm 0.01$ & $2.48 \pm 0.39$ \\
$\mathrm{D}$ & $20 / 32 / 32 / 16$ & None & $4.94 \pm 0.01$ & $4.36 \pm 0.04$ \\
$\mathrm{E}$ & $23 / 30.8 / 30.8 / 15.4$ & None & $4.82 \pm 0.01$ & $5.57 \pm 0.33$ \\
$\mathrm{~F}$ & $5 / 38 / 38 / 19$ & $0.05 \mathrm{~g} / \mathrm{mL}$ & $5.68 \pm 0.00$ & Size under detection \\
& & & & limit \\
$\mathrm{G}$ & $10 / 36 / 36 / 18$ & $0.05 \mathrm{~g} / \mathrm{mL}$ & $5.60 \pm 0.01$ & $1.07 \pm 0.06$ \\
$\mathrm{H}$ & $15 / 34 / 34 / 17$ & $0.05 \mathrm{~g} / \mathrm{mL}$ & $5.40 \pm 0.01$ & $2.19 \pm 0.15$ \\
$\mathrm{I}$ & $20 / 32 / 32 / 16$ & $0.05 \mathrm{~g} / \mathrm{mL}$ & $5.38 \pm 0.01$ & $2.88 \pm 0.21$ \\
$\mathrm{~J}$ & $23 / 30.8 / 30.8 / 15.4$ & $0.05 \mathrm{~g} / \mathrm{mL}$ & $5.32 \pm 0.01$ & $4.51 \pm 0.15$ \\
\hline
\end{tabular}




\subsubsection{In vitro permeation studies}

\subsubsection{Skin preparation}

Human skin samples (chest and abdominal regions) were purchased from National Disease Research Interchange (NDRI, Philadelphia, PA, USA). Subcutaneous fatty tissues were removed from skin using a lancet after soaking the skin in a $60{ }^{\circ} \mathrm{C}$ water bath for 1 minute. ${ }^{125}$ Thereafter, skin samples were washed with di-ionized water. Prior to the actual permeation study, the fat free skin was stored refrigerated at $4{ }^{\circ} \mathrm{C}$.

\subsubsection{In vitro diffusion experiments}

To investigate KI formulation diffusion through the skin, Franz cells (PermeGear Inc, Hellertown, PA, USA) were utilized. The receptor volume of each cell was $5 \mathrm{~mL}$ and the diffusion area was $0.64 \mathrm{~cm}^{2}$. Prior to mounting the skin samples, each receptor was filled with $5 \mathrm{~mL}$ di-ionized water. Successively the skin samples were clamped in between the receptor (down) and donor (up) holding the stratum corneum side up. Prior to the experiment, the jacketed receptor was kept for 1 hour at $37^{\circ} \mathrm{C}$ using a water bath with magnetic stirring. Afterwards, $1 \mathrm{~mL}$ of each selected formulation with $50 \mathrm{mg} / \mathrm{mL} \mathrm{KI}$ was loaded to the donor compartment and each donor cell was sealed with Parafilm ${ }^{\circledR}$ to avoid the evaporation of formulation components. A KI solution $(1 \mathrm{~mL}$ of $50 \mathrm{mg} / \mathrm{mL}$ solution) was used as the control. Then, $250 \mu \mathrm{L}$ of the aqueous liquid were withdrawn from the sampling port of receptor and diluted with di-ionized water to $5 \mathrm{~mL}$ at different time points ( 0 h, 2 h, 4 h, 6 h, 8 h, 12 h, and 24 h). Simultaneously, equal volume of diionized water was replaced into the liquid. The diluted samples were filtered through $0.45 \mu \mathrm{m}$ Millex ${ }^{\circledR}$ filter (Millipore, Billerica, MA, USA) and analyzed using Orion iodide selective electrode (Thermo Fisher Scientific Inc, Pittsburgh, PA, USA). Three replicates were carried out for each selected formulation.

The concentration of iodide in the receptor at every time point was calculated after incorporating the dilution factor. Then the cumulative amount of KI permeated across the skin per unit area $\left(\mathrm{mg} / \mathrm{cm}^{2}\right)$ was obtained by using the following Equation 5-1.

$$
\mathrm{Q}_{\mathrm{n}}=\frac{\mathrm{C}_{\mathrm{n}} \times \mathrm{V}_{0}+\sum_{\mathrm{i}=1}^{\mathrm{n}-1} \mathrm{C}_{\mathrm{i}} \times \mathrm{V}_{\mathrm{i}}}{\mathrm{S}}
$$

Where $\mathrm{C}_{\mathrm{n}}$ is the undiluted sample concentration (liquid concentration in the receptor) at $\mathrm{n}^{\text {th }}$ sampling time point, $\mathrm{C}_{\mathrm{i}}$ is undiluted sample concentration (liquid concentration in the receptor) at $i^{\text {th }}$ sampling time point, $\mathrm{V}_{0}$ is the receptor volume $(5 \mathrm{~mL}), \mathrm{V}_{\mathrm{i}}$ is the sampling volume $(250 \mu \mathrm{L})$, and $S$ is diffusion area $\left(0.64 \mathrm{~cm}^{2}\right)$. All $Q_{n}$ values at each time point were plotted as a function of time. The steady state flux $\left(\mathrm{J}_{\mathrm{ss}}, \mathrm{mg} / \mathrm{cm}^{2}\right.$ per hour $)$ was calculated for every formulation. $\mathrm{J}_{\mathrm{ss}}$ is the slope of linear portion of cumulative iodide amounts. 


\subsubsection{Accelerated microstructure stability testing}

\subsubsection{Centrifugation}

Selected formulations $(2 \mathrm{~mL})$ with and without KI were centrifuged at 13,000 rpm $(13,793 \mathrm{~g})$ for 30 minutes using Eppendorf 5415C centrifuge.

\subsubsection{Thermal stability}

Selected formulations $(20 \mathrm{~mL})$ were stored in sealed vials at a $40{ }^{\circ} \mathrm{C}$ stability chamber for 4 weeks. Three replicates were carried out for each formulation.

\subsubsection{Chemical stability}

Potassium iodide-starch test paper was utilized to test the existence of iodine. Four standard iodine solutions $(2.5 \mathrm{E}-2 \mathrm{mg} / \mathrm{mL}, 2.5 \mathrm{E}-1 \mathrm{mg} / \mathrm{mL}, 5 \mathrm{E}-1 \mathrm{mg} / \mathrm{mL}, 1 \mathrm{mg} / \mathrm{mL})$ were prepared by dissolving iodine in ethanol/water $(50 / 50 \mathrm{w} / \mathrm{w})$ solution. These standards were utilized as indicators for any presence of iodine in microemulsion formulations. Formulation J ( $23 \%$ water microemulsion with $50 \mathrm{mg} / \mathrm{mL} \mathrm{KI}$ ) was selected to observe the presence of iodine as a function of time ( 0 week, 2 weeks, and 4 weeks) at ambient temperature. The test paper was dipped into the selected microemulsion for 30 seconds, followed by washing with a small volume of water to provide the aqueous environment for the triiodide starch reaction.

\subsection{Results}

\subsubsection{Construction of pseudo-ternary phase diagrams}

Non-ionic surfactants are utilized in topical formulations because they are less irritating to skin and have less- tendency to cause allergic reactions. ${ }^{22}$ Moreover, it has been reported that non-ionic surfactants with poly-ethoxylated groups and/or residues may contain peroxides which accelerate the oxidation of iodide to iodine. ${ }^{126,127,128}$ For this reason, Span 20 (HLB 8.6) was selected as the surfactant since it contains no ethoxylated groups. In addition, Span 20 acts as a penetration enhancer which fluidizes the intercellular lipid bilayer. ${ }^{129}$ However, the emulsification capacity of Span 20 is limited since it has a relatively small polar group and a short nonpolar carbon chain. In order to obtain a stable microemulsion system at relatively high water content, ethanol was selected as a cosurfactant. Ethanol has been widely applied as a penetration enhancer. ${ }^{23}$ Capryol 90® (HLB 6), a common penetration enhancer, was selected as the oil phase. ${ }^{130,131}$ The formation of microemulsion is dependent on the assumption that Capryol $90 \AA$ interacts with the hydrophobic chain of Span 20 in the presence of ethanol to lower the oil water interface. Pseudo-ternary phase diagrams were constructed to evaluate the miscibility of the microemulsion components. ${ }^{132}$ Mixtures of Capryol $90 \AA$, Span 20, ethanol and water with various $\mathrm{K}_{\mathrm{m}}(\operatorname{Span} 20 /$ ethanol, w/w) ratios are depicted in 
Figure 5-1. $A_{T}$ represents the monophasic area (solubilization capacity) and it tends to increase as the amount of span to ethanol increases in the formulations $\left(\mathrm{K}_{\mathrm{m}}: 4: 1\right.$, $\left.\mathrm{A}_{\mathrm{T}}: 13.1 ; \mathrm{K}_{\mathrm{m}}: 1: 1, \mathrm{~A}_{\mathrm{T}}: 23.8 ; \mathrm{K}_{\mathrm{m}}: 1: 4, \mathrm{~A}_{\mathrm{T}}: 30.4 ; \mathrm{K}_{\mathrm{m}}: 1: 9, \mathrm{~A}_{\mathrm{T}}: 32.0\right)$.

\subsubsection{Droplet size and $\mathrm{pH}$ measurements}

Dynamic light scattering technique measures droplet size through direct measurement of the droplet diffusion coefficient in a dispersed medium undergoing Brownian motion then the droplet size is obtained from the Stokes-Einstein equation. In the selected formulations, surfactant, cosurfactant, and oil form the external phase, while water (aqueous core) is the internal phase. The existing boundaries between oil and aqueous core are composed of the polar parts of Span 20, the water and ethanol. The results for droplet size are depicted in Table 5-1. Mean droplet size was not measurable for formulation containing 5\% water by dynamic light scattering. These formulations may resemble cosolvent systems. It is possible that when water content in the system is low, water molecules can stay separate without the forming an aqueous droplets. As the water content increases to $10 \%$, the average droplet size becomes more than $1 \mathrm{~nm}$. It was observed that as water content further increased, droplet size of microemulsion increased also.

Surprisingly, the addition of KI to microemulsions shrinks water droplets. This is due to the salting-in effect which occurs between inorganic anionic ions such as iodide ions and water molecules. Thus, anionic ion makes water less polar and makes the organic components dissolve more readily into internal aqueous clusters. ${ }^{133}$ A schematic demonstration of microemulsion with and without KI is shown in Figure 5-3.

It is known that iodide, a member of Hofmeister ion series, tends to increase the solubility of nonpolar components in aqueous solvent by decreasing the surface tension between water and organic molecules. ${ }^{134}$ Thus, the polar region (water content in the core) diminishes and the existing boundary between polar and non-polar molecules shrinks consequently, which causes the droplet size to decrease.

The $\mathrm{pH}$ values of all selected formulations were physiologically acceptable for topical uses. It was observed that the $\mathrm{pH}$ decreased from 5.20 to 4.82 when the amount of water increased from 5\% to $23 \%$ (Table 5-1). However, after the incorporation of KI, pH values increased slightly from 5.68 to 5.32 (5\% to $23 \%$ water content), as shown in

\section{Table 5-1.}

\subsubsection{Viscosity measurements}

Viscosity of multi-component systems is a polynomial function which depends on the concentrations of water, surfactant, cosurfactant, and oil in each formulation. ${ }^{135}$ Figure 5-4 depicts the dynamic viscosity values obtained for all tested formulations in the presence and absence of KI as it relates to water contents. The viscosity values for 

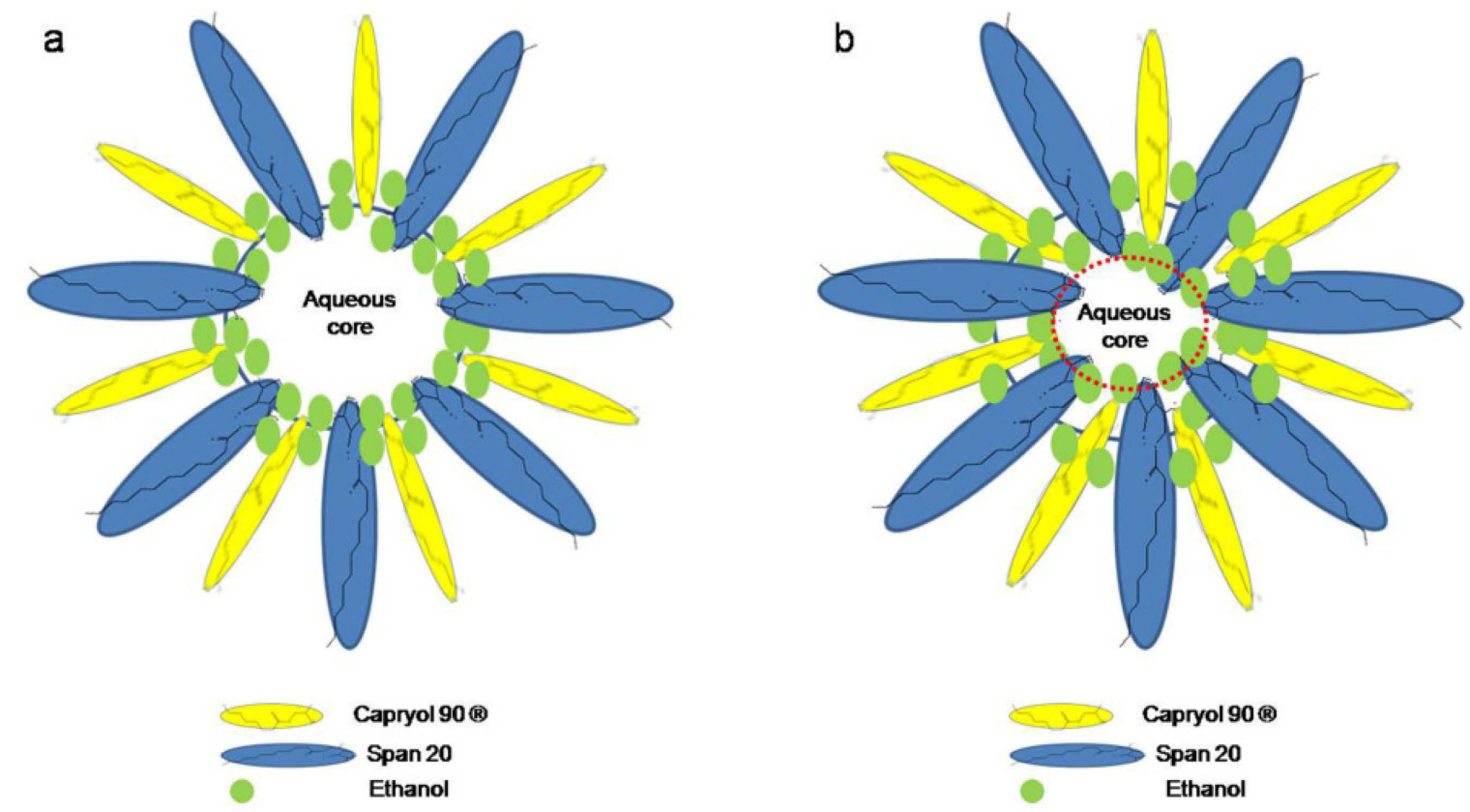

Figure 5-3. A schematic demonstration for the water interface of microemulsions.

(a) microstructure of microemulsion without KI; (b) microstructure of microemulsion with KI. The internal domain represents the water content. 


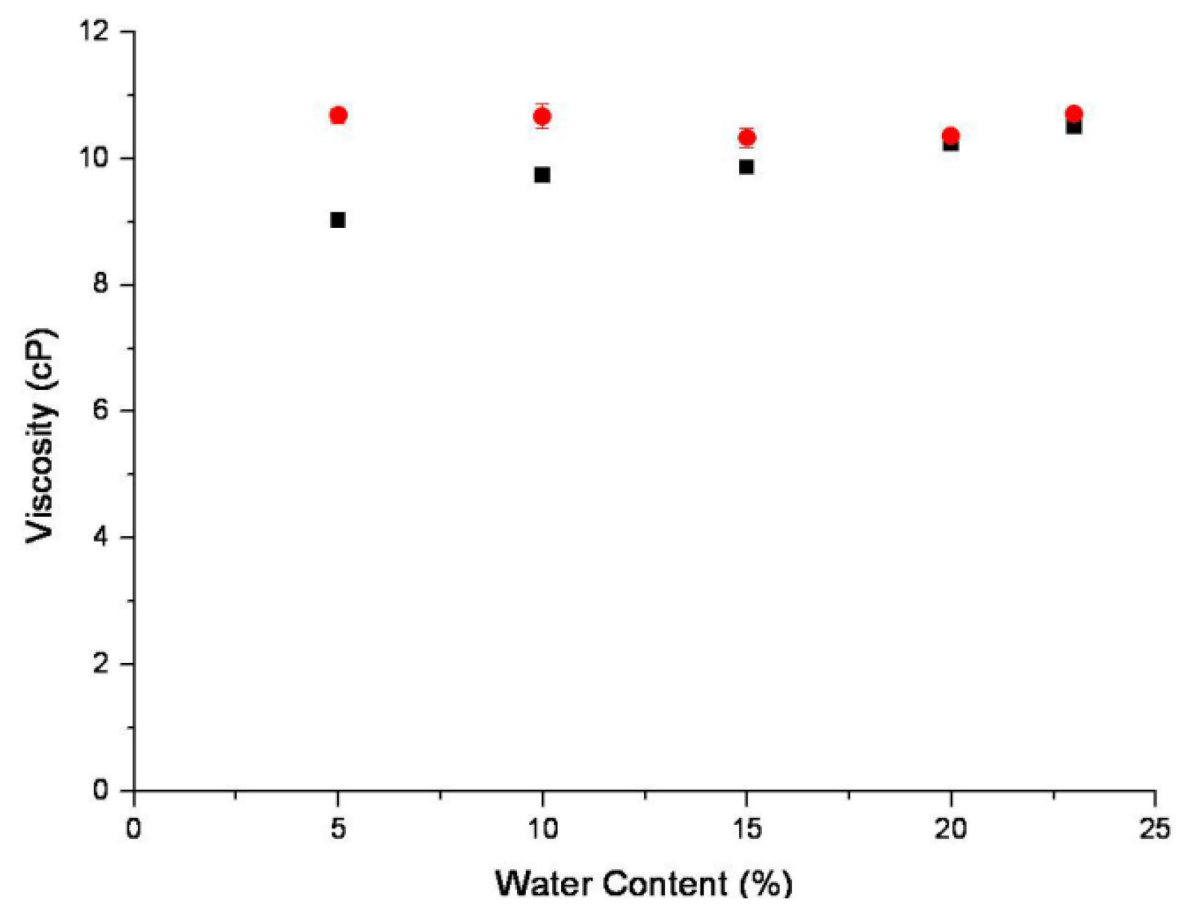

Figure 5-4. The change of dynamic viscosity as a function of water.

Water is the aqueous phase of microemulsions along the dilution line L20.

Microemulsions without KI loading are labeled as $\mathbf{-}$; microemulsions with KI loading are labeled as $\bullet$. 
formulations without $\mathrm{KI}$ were relatively low and ranged between 9 to $11 \mathrm{cPoise}$. It was observed for the aforementioned formulations, viscosity increased as the water content increased from 5 to $23 \%$. The observed increase in the viscosity with the water content is dependent upon the increase of the dispersant phase droplet's volume and the increase in the frequency of collisions between the water droplets in W/O microemulsion system. ${ }^{136}$

It was noted that after the addition of KI the viscosity of the formulations (Figure 5-4) increased slightly (10 to 11 cPoise). The increase of viscosity is not only due to more densed internal aqueous phase, but also due to the increase in the formation of aqueous transition clusters where iodide ions cause aqueous phase to become more hydrophobic and free to move. ${ }^{137}$

\subsubsection{Conductivity measurements}

The influence of water content on conductivities of selected formulations is presented in Figure 5-5. The main graph relates overall ions conductivity of water content from 0 to $24.5 \%$.

In general, when water molecules are dispersed in an oil phase at a small volume fraction, droplets are separated from each other and exhibits minimum interactions and liquid conductivity is low. Further addition of water increases the total number of aqueous droplets which can increase the formation and deformation dynamics of the transient clusters (aggregation of water molecules) to increase liquid conductivity. The cluster formation and deformation process is described in three steps: fusion, mass transfer and fission. ${ }^{138}$ Transient clusters have significant influence on increasing the conductivity. The transient collisions of water droplets provide water channels where ions hop from one droplet to the other. ${ }^{139}$ Therefore, a rapid increase in conductivity up to $23 \%$ water content was also observed. This observation is consistent with other researchers who described similar conductivity behavior in W/O microemulsion systems. ${ }^{140}$ Remarkably, the addition of $\mathrm{KI}$ into the selected formulations enhanced conductivities more than 50 -fold as compared to microemulsions without KI. This observation is consistent with the quantitative charge fluctuation model where the aqueous channels created by the transient clusters contain more dissociated ions which are able to facilitate the overall conductivity tremendously. ${ }^{141}$

\subsubsection{In vitro microemulsion skin permeation studies}

Cumulative amounts of iodide that permeated through human skin over 24 hours for selected formulations are depicted in Figure 5-6. Results indicated that at the end of 24 hours all KI formulations with different water contents $(5 \%, 10 \%, 15 \%, 20 \%$, and $23 \%$ ) had significantly better iodide permeation through skin as compared to the control sample (KI solution; student paired t-test, $\mathrm{P}<0.05$ ).

Formulation F exhibited the lowest cumulative amount of iodide that permeated 


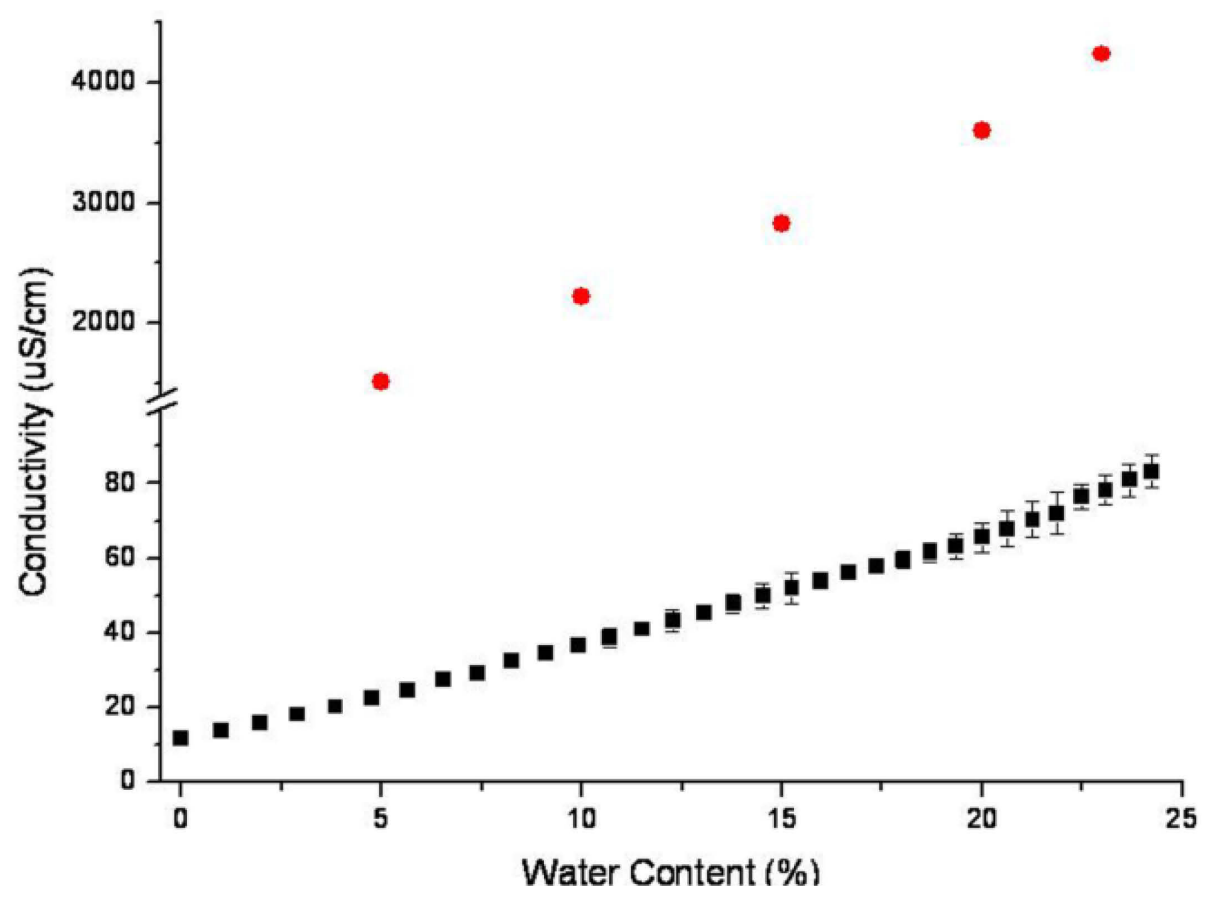

Figure 5-5. Conductivity of microemulsion formulations along dilution line L20 versus water content.

Microemulsions without KI loading are labeled as $\mathbf{\square}$; microemulsions with KI loading are labeled as $\bullet$. 


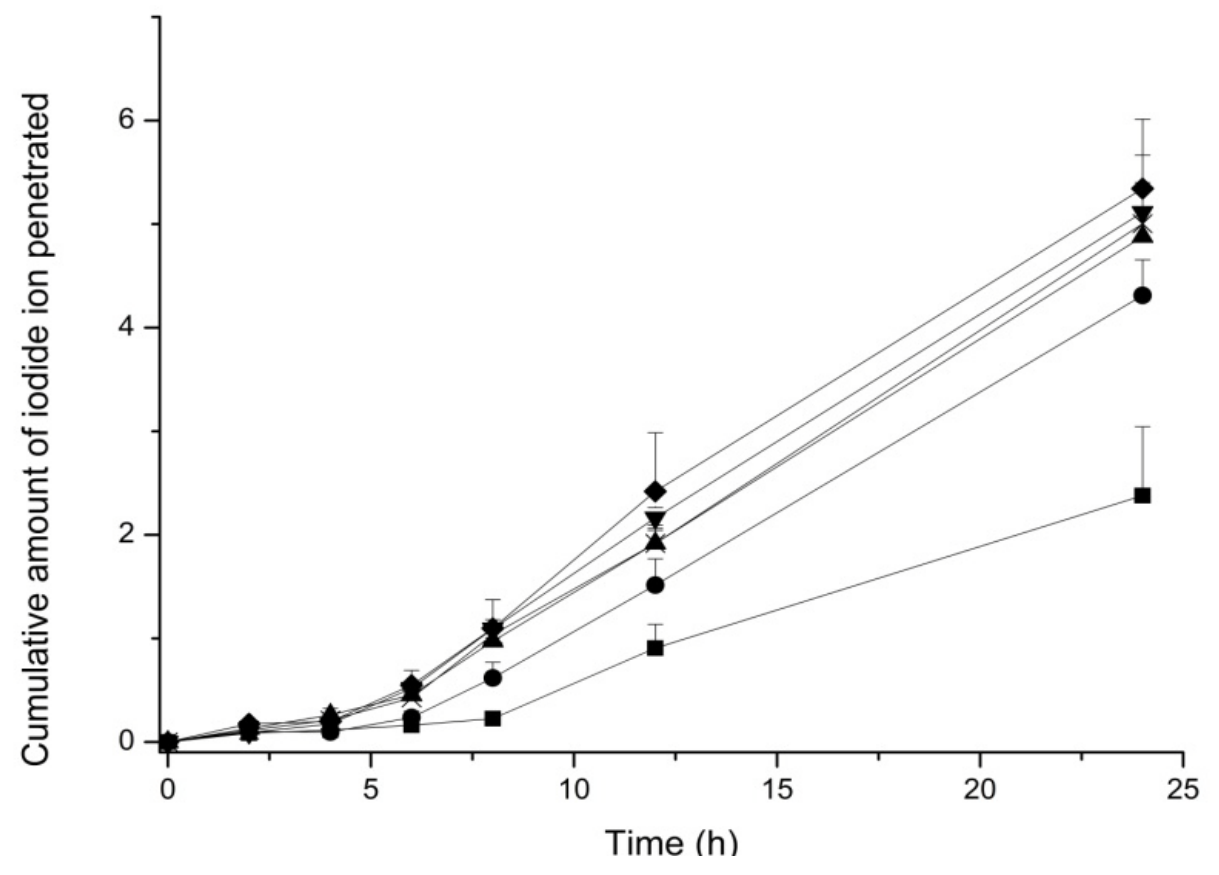

Figure 5-6. Permeation profiles of KI formulations.

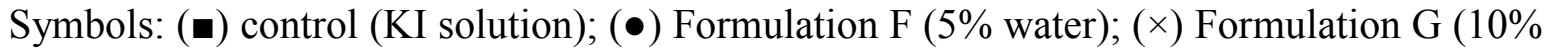
water); ( $\boldsymbol{\nabla})$ Formulation H (15\% water); ( $\boldsymbol{\Delta}$ ) Formulation I (20\% water); ( $)$ Formulation $\mathrm{J}$ (23\% water). 
the skin at the end of 24 hours out of the five selected formulations ( $\mathrm{F}$ to $\mathrm{J}$ ). It permeated about 2 times the amount of KI as compared to the solution after 24 hours. On the other hand, Formulation $\mathrm{J}$ was the most effective formulation for iodide permeation study (about 2.5 times of KI solutions) after 24 hours. Statistically, iodide permeation at the end of 24 hour for Formulation J (23\% water content) was significantly higher (student paired $\mathrm{t}$ test, $\mathrm{P}<0.05)$ as compared to Formulation $\mathrm{F}(5 \%$ water content), but had no significant difference compared to Formulation $\mathrm{G}$ ( $10 \%$ water content), $\mathrm{H}$ (15\% water content), and I ( $20 \%$ water content) (student paired t test, $\mathrm{P}>0.05)$.

Values of steady-state flux and cumulative amounts of iodide that permeated are listed in Table 5-2. Steady-state flux values $\left(\mathrm{J}_{\mathrm{ss}}\right)$ indicates that selected formulations ( $\mathrm{F}$ to J) had a significant better permeation of iodide compared to KI solution. These results indicate that organic components in microemulsion formulations act as penetration enhancers. They potentially modify the lipid structure within the stratum corneum and make it looser and more porous for iodide permeation. Likewise, larger amount of water in w/o microemulsion could influence iodide permeability to a higher extent since Formulation $\mathrm{J}$ ( $23 \%$ water content) had the highest flux rate $0.266 \pm 0.037 \mathrm{mg} / \mathrm{cm}^{2} / \mathrm{h}$. In the presence of water, skin is hydrated and exists in a swollen state, thus more void spaces within the skin create wider diffusion channels. ${ }^{142}$ In summary, the permeation profile of iodide within microemulsion formulations is affected by a combination of factors including permeation enhancement and skin hydration.

\subsubsection{Accelerated microstructure stability testing}

Centrifugational forces accelerate physical instability of microemulsions and lead to turbidity and phase separation. ${ }^{143}$ Brownian motion maintains droplets' kinetic energy which causes irregular movements of small droplets, so it prevents droplet settling. Additionally low interfacial tension and droplets kinetic energy lead to inhibition of creaming, sedimentation, flocculation, and coalescence. ${ }^{144}$

All selected formulations (A-J) in Table 5-1 in the presence and the absence of KI had no phase separation and clarity change by the end of 30 minutes under high centrifugational forces $(13,000 \mathrm{rpm}, 13,793 \mathrm{~g})$ which is a sign of the strong physical stability of formulations.

Thermal stability testing under $40{ }^{\circ} \mathrm{C}$ showed no turbidity by the end of three weeks, thus lending further support to the physical stability of the microemulsion under thermal stress.

Chemical stability was performed by exploring the presence of degradation product iodine. Iodine reacts with starch in the presence of iodide and expresses blueblack color. So we utilized iodide-starch test paper as an indicator to detect the existence of iodine. A series of iodine solution standards with different concentrations (Figure 5-7) were prepared at $0.05 \%(2.5 \mathrm{E}-2 \mathrm{mg} / \mathrm{mL}$ iodine $), 0.5 \%(2.5 \mathrm{E}-1 \mathrm{mg} / \mathrm{mL}$ iodine $), 1 \%(5 \mathrm{E}-1$ $\mathrm{mg} / \mathrm{mL}$ iodine $)$, and $2 \%(1 \mathrm{mg} / \mathrm{mL}$ iodine $)$. The rational for preparing these four iodine 
Table 5-2. Cumulative permeated iodide $\left(Q_{24}\right)$ and flux at steady-state (Jss) $\left(\mathrm{mg} / \mathrm{cm}^{2} / \mathrm{h}\right)$ of selected formulations.

\begin{tabular}{|c|c|c|}
\hline $\begin{array}{c}\text { Formulation } \\
(0.05 \mathrm{~g} / \mathrm{mL} \text { KI })\end{array}$ & $\begin{array}{c}\text { Cumulative Permeated Iodide } \\
\left(\mathrm{Q}_{24}\right)\left(\mathrm{mg} / \mathrm{cm}^{2}\right)\end{array}$ & $\begin{array}{c}\text { Flux at Steady-state } \\
\left(J_{\text {ss }}\right)\left(\mathrm{mg} / \mathrm{cm}^{2} / \mathrm{h}\right)\end{array}$ \\
\hline Solution & $2.38 \pm 0.66$ & $0.127 \pm 0.036$ \\
\hline $\mathrm{F}$ & $4.31 \pm 0.34$ & $0.228 \pm 0.014$ \\
\hline $\mathrm{G}$ & $5.00 \pm 0.66$ & $0.252 \pm 0.038$ \\
\hline $\mathrm{H}$ & $5.11 \pm 0.29$ & $0.254 \pm 0.014$ \\
\hline $\mathrm{I}$ & $4.88 \pm 0.50$ & $0.245 \pm 0.029$ \\
\hline $\mathrm{J}$ & $5.35 \pm 0.53$ & $0.266 \pm 0.037$ \\
\hline
\end{tabular}
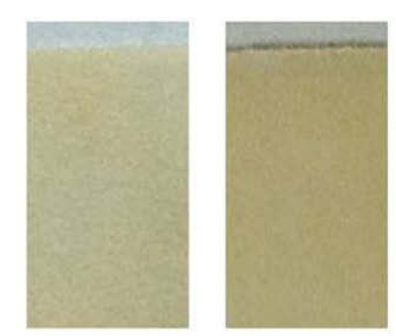

0 Week 2 Week

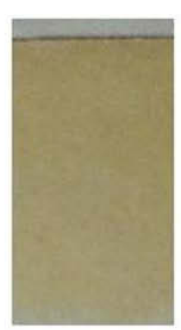

4 Week

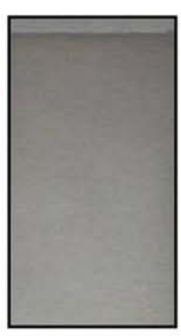

$0.05 \%$

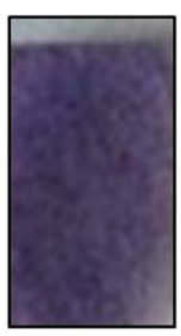

$0.5 \%$

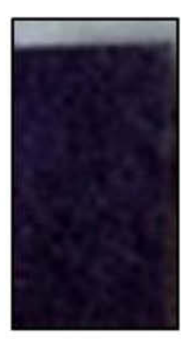

$1 \%$

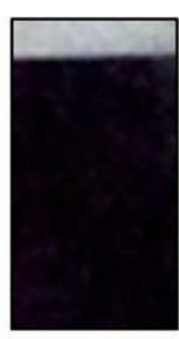

$2 \%$

Figure 5-7. Test papers for sample stability test.

From left to right: sample at 0 week, sample at 2 weeks, sample at 4 weeks, sample with $2.5 \mathrm{E}-5 \mathrm{~g} / \mathrm{mL}$ iodine, sample with $2.5 \mathrm{E}-4 \mathrm{~g} / \mathrm{mL}$ iodine, sample with $5 \mathrm{E}-4 \mathrm{~g} / \mathrm{mL}$ iodine, and sample with $1 \mathrm{E}-3 \mathrm{~g} / \mathrm{mL}$ iodine. 
standards was related to the assumption that if the percentages of degradation of the initial concentration of potassium iodide in microemulsion $(50 \mathrm{mg} / \mathrm{mL})$ were $0.05 \%$, $0.5 \%, 1 \%$ and $2 \%$ then the concentrations of iodine that should be formed will be $2.5 \mathrm{E}-2$, $2.5 \mathrm{E}-1,5 \mathrm{E}-1$ and $1 \mathrm{mg} / \mathrm{mL}$ respectively.

We observed that with increasing iodine concentration the blue-black color became more intense. Samples were collected over 1 month period (Figure 5-7). At the end of one month, the absence of blue-dark color in iodide-starch test paper indicates the percentage of degradation product iodine is much less than $0.5 \%$. Thus, we report with confidence that KI microemulsion with $23 \%$ water is chemically stable for at least one month.

\subsection{Discussion}

Since, the dose requirement for iodide is 150 micrograms per day, a transdermal delivery of microemulsion iodide is feasible. In this study, we explored a W/O microemulsion as a vehicle which could potentially improve the permeation of iodide ion through skin. In this system, Capryol 90®, Span 20, ethanol, and water were combined at appropriate ratios. Despite the fact that other surfactants with poly-oxyethylene groups have better solubilization capacity than Span 20, these types of surfactants are not selected since the residues of peroxide originated from poly-oxyethylene groups may lead to the oxidation of iodide. Thus to stabilize the microemulsion, a co-surfactant is necessary to further decrease the interfacial tension between the oil phase and the aqueous phase. We have selected ethanol as the cosurfactant for this system since it is relatively polar and miscible with water and considered safe at a major content of less than $40 \% \mathrm{w} / \mathrm{w}$. The addition of potassium iodide did not show any evidence of microemulsion destabilization. Iodide ion disrupts water-water interactions and makes water more hydrophobic thus forcing water dissolve more organic components including ethanol, Span 20, and Capryol $90 \AA$ into the aqueous phase. As a result, the internal droplet size of water decreases. This phenomenon was also explained by other authors who illustrated that iodide ions adsorb and interact to the inter-phase. ${ }^{133}$ On the other hand, cationic ions such as potassium have subtle effect on microemulsion system.

A perspective application for this iodide microemulsion is envisioned to be in the therapy for specialized patients who suffer from iodide deficiency or who suffer from short bowel syndrome where oral absorption is limited. Currently patients with this illness can only rely on parental routes since the majority of nutritional elements including iodide may be difficult to get absorbed by GI tract. Therefore, a satisfactory transdermal delivery system may provide a new advent for the therapy of this disease. Transdermal delivery of iodide could serve as the first front for the transdermal delivery of nutrients. Although microemulsion is a non-toxic and non-invasive colloidal formulation, there are still limitations for topical use because of the poor adherence on the skin. A patch system of microemulsion will provide the solution for this problem. Such a patch will maintain the microemulsion entrapped behind the adhesive layer and the membrane and will control the release of the ions. 


\subsection{Conclusion}

In summary, several w/o microemulsion formulations were prepared and fully characterized and evaluated. The physicochemical characterizations included: $\mathrm{pH}$, droplet size, viscosity, conductivity, and stability. These studies indicated that the selected formulations were stable and compatible with iodide ions. The permeation studies in human skin showed that microemulsion enables the iodide ions to diffuse through the skin. Further development of this work will be to incorporate the microemulsions into a patch which can be applied more conveniently in clinical treatment. 


\section{LIST OF REFERENCES}

1. Strickley RG, Iwata Q, Wu S, Dahl TC. Pediatric drugs-a review of commercially available oral formulations. J Pharm Sci 2008;97:1731-74.

2. Brown D. Orally disintegrating tablets: taste over speed. Drug Delivery Tech 2001;3:58-61.

3. Sandri G, Maria Cristina B, Ferrari F, Rossi S, Caramella C. Differentiating factors between oral fast-dissolving technologies. Am J Drug Delivery 2006;4:249-62.

4. Sastry SV, Nyshadham JR, Fix JA. Recent technological advances in oral drug delivery - a review. Pharm Sci Tech Today 2000;3:138-45.

5. $\mathrm{Xu} \mathrm{J}$, Bovet $\mathrm{LL}$, Zhao K. Taste masking microspheres for orally disintegrating tablets. Int J Pharm 2008;359:63-9.

6. Khan S, Kataria P, Nakhat P, Yeole P. Taste masking of ondansetron hydrochloride by polymer carrier system and formulation of rapid-disintegrating tablets. AAPS PharmSciTech 2007;8:127-33.

7. Dixit RP, Puthli SP. Oral strip technology: overview and future potential. J Control Release 2009;139:94-107.

8. Cilurzo F, Cupone I, Minghetti P, Buratti S, Selmin F, Gennari C, et al. Nicotine fast dissolving films made of maltodextrins: a feasibility study. AAPS PharmSciTech 2010;11:1511-7.

9. Cilurzo F, Cupone IE, Minghetti P, Selmin F, Montanari L. Fast dissolving films made of maltodextrins. Eur J Pharm Biopharm 2008;70:895-900.

10. Dinge A, Nagarsenker M. Formulation and evaluation of fast dissolving films for delivery of triclosan to the oral cavity. AAPS PharmSciTech 2008;9:349-56.

11. Gaisford S, Verma A, Saunders M, Royall PG. Monitoring crystallisation of drugs from fast-dissolving oral films with isothermal calorimetry. Int J Pharm 2009;380:105-11.

12. Zhou B, Carvajal T, Cheng J, Nivens D, Pinal R. Characterization of strip films as carriers of micro- and submicron drug particles. AAPS Annual Meeting Exposition, Los Angeles, CA 2009.

13. Lennartz P, Mielck JB. Minitabletting: improving the compactability of paracetamol powder mixtures. Int J Pharm 1998;173:75-85. 
14. Thomson SA, Tuleu C, Wong ICK, Keady S, Pitt KG, Sutcliffe AG. Minitablets: new modality to deliver medicines to preschool-aged children. Pediatrics 2009;123:235-8.

15. Tissen C, Woertz K, Breitkreutz J, Kleinebudde P. Development of mini-tablets with $1 \mathrm{~mm}$ and 2mm diameter. Int J Pharm 2011;416:164-70.

16. Saettone MF, Chetoni P, Bianchi LM, Giannaccini B, Conte U, Sangalli ME. Controlled release of timolol maleate from coated ophthalmic mini-tablets prepared by compression. Int J Pharm 1995;126:79-82.

17. Kayser O, Olbrich C, Yardley V, Kiderlen AF, Croft SL. Formulation of amphotericin B as nanosuspension for oral administration. Int J Pharm 2003;254:73-5.

18. Keck CM, Müller RH. Drug nanocrystals of poorly soluble drugs produced by high pressure homogenisation. Eur J Pharm Biopharm 2006;62:3-16.

19. Shegokar R, Müller RH. Nanocrystals. Industrially feasible multifunctional formulation technology for poorly soluble actives. Int J Pharm 2010;399:129-39.

20. Cevc G, Vierl U. Nanotechnology and the transdermal route: a state of the art review and critical appraisal. J Control Release 2010;141:277-99.

21. Danielsson I, Lindman B. The definition of microemulsion. Colloids Surf 1981;3:391-2.

22. Kogan A, Garti N. Microemulsions as transdermal drug delivery vehicles. Adv Colloid Interface Sci 2006;123:369-85.

23. Williams AC, Barry BW. Penetration enhancers. Adv Drug Deliv Rev 2004;56:603-18.

24. Sohi H, Sultana Y, Khar RK. Taste masking technologies in oral pharmaceuticals: recent developments and approaches. Drug Dev Ind Pharm 2004;30:429-48.

25. Patel A, Vavia P. Preparation and evaluation of taste masked famotidine formulation using drug/ $\beta$-cyclodextrin/polymer ternary complexation approach. AAPS PharmSciTech 2008;9:544-50.

26. Funasaki N, Kawaguchi R, Hada S, Neya S. Ultraviolet spectroscopic estimation of microenvironments and bitter tastes of oxyphenonium bromide in cyclodextrin solutions. J Pharm Sci 1999;88:759-62. 
27. Redenti E, Szente L, Szejtli J. Cyclodextrin complexes of salts of acidic drugs: Thermodynamic properties, structural features, and pharmaceutical applications. J Pharm Sci 2001;90:979-86.

28. Agarwal R, Mittal R, Singh A. Studies of ion-exchange resin complex of chloroquine phosphate. Drug Dev Ind Pharm 2000;26:773-6.

29. Cerea M, Zheng W, Young CR, McGinity JW. A novel powder coating process for attaining taste masking and moisture protective films applied to tablets. Int $\mathrm{J}$ Pharm 2004;279:127-39.

30. Athanikar NK, Chiou WL. Chlorpheniramine. II. effect of the first-pass metabolism on the oral bioavailability in dogs. J Pharmacokinet Pharmacodyn 1979; 7:383-96.

31. Mohanan D, Gander B, Kündig TM, Johansen P. Encapsulation of antigen in poly(d,1-lactide-co-glycolide) microspheres protects from harmful effects of $\gamma$ irradiation as assessed in mice. Eur J Pharm Biopharm 2012;80:274-81.

32. O'Donnell PB, McGinity JW. Preparation of microspheres by the solvent evaporation technique. Adv Drug Deliver Rev 1997;28:25-42.

33. Bozkir A, Saka OM. Formulation and investigation of 5-FU nanoparticles with factorial design-based studies. II Farmaco 2005;60:840-6.

34. Freiberg S, Zhu XX. Polymer microspheres for controlled drug release. Int J Pharm 2004;282:1-18.

35. Kim KS, Park SJ. Characterization and release behaviors of porous PCL/Eudragit RS microcapsules containing tulobuterol. Colloids Surf B 2010;76:404-9.

36. Dillen K, Vandervoort J, Van den Mooter G, Verheyden L, Ludwig A. Factorial design, physicochemical characterisation and activity of ciprofloxacin-PLGA nanoparticles. Int J Pharm 2004;275:171-87.

37. Sugimoto M, Narisawa S, Matsubara K, Yoshino H, Nakano M, Handa T. Effect of formulated ingredients on rapidly disintegrating oral tablets prepared by the crystalline transition method. Chem Pharm Bull 2006;54:175-80.

38. Albertini B, Cavallari C, Passerini N, Voinovich D, González-Rodrí guez ML, Magarotto L, et al. Characterization and taste-masking evaluation of acetaminophen granules: comparison between different preparation methods in a high-shear mixer. Eur J Pharm Sci 2004;21:295-303. 
39. Sugao H, Yamazaki S, Shiozawa H, Yano K. Taste masking of bitter drug powder without loss of bioavailability by heat treatment of wax-coated microparticles. J Pharm Sci 1998;87:96-100.

40. Yu L. Pharmaceutical quality by design: product and process development, understanding, and control. Pharm Res 2008;25:781-91.

41. Wang YM, Sato H, Adachi I, Horikoshi I. Optimization of the formulation design of chitosan microspheres containing cisplatin. J Pharm Sci 1996;85:1204-10.

42. Fonner DE, Buck JR, Banker GS. Mathematical optimization techniques in drug product design and process analysis. J Pharm Sci 1970;59:1587-96.

43. Youm I, Murowchick JB, Youan B-BC. Entrapment and release kinetics of furosemide from pegylated nanocarriers. Colloids Surf B 2012;94:133-42.

44. Zhu W, Yu A, Wang W, Dong R, Wu J, Zhai G. Formulation design of microemulsion for dermal delivery of penciclovir. Int J Pharm 2008;360:184-90.

45. Sun Y, Peng Y, Chen Y, Shukla AJ. Application of artificial neural networks in the design of controlled release drug delivery systems. Adv Drug Deliver Rev 2003;55:1201-15.

46. Takayama K, Fujikawa M, Obata Y, Morishita M. Neural network based optimization of drug formulations. Adv Drug Deliver Rev 2003;55:1217-31.

47. Lipinski CA. Poor solubility - an industry wide problem in drug discovery. Am Pharm Rev 2002;5:82-85.

48. Shah V, Konecny J, Everett R, McCullough B, Noorizadeh AC, Skelly J. In vitro dissolution profile of water-insoluble drug dosage forms in the presence of surfactants. Pharm Res 1989;6:612-8.

49. Leuner C, Dressman J. Improving drug solubility for oral delivery using solid dispersions. Eur J Pharm Biopharm 2000;50:47-60.

50. Kobayashi Y, Ito S, Itai S, Yamamoto K. Physicochemical properties and bioavailability of carbamazepine polymorphs and dihydrate. Int J Pharm 2000;193:137-46.

51. Challa R, Ahuja A, Ali J, Khar R. Cyclodextrins in drug delivery: an updated review. AAPS PharmSciTech 2005;6:329-57.

52. Berge S, Bighley L, Monkhouse D. Pharmaceutical salts. J Pharm Sci 1977;66:119. 
53. Neslihan GR, Benita S. Self-emulsifying drug delivery systems (SEDDS) for improved oral delivery of lipophilic drugs. Biomed Pharmacother 2004;58:173-82.

54. Keck CM, Müller RH. Drug nanocrystals of poorly soluble drugs produced by high pressure homogenisation. Eur J Pharm Biopharma 2006;62:3-16.

55. Hecq J, Deleers M, Fanara D, Vranckx H, Amighi K. Preparation and characterization of nanocrystals for solubility and dissolution rate enhancement of nifedipine. Int J Pharm 2005;299:167-77.

56. Shegokar R, Müller RH. Nanocrystals: industrially feasible multifunctional formulation technology for poorly soluble actives. Int J Pharm 2010;399:129-39.

57. Hu J, Johnston K, Williams R. Nanoparticle engineering processes for enhancing the dissolution rates of poorly water soluble drugs. Drug Dev Ind Pharm 2004;30:233-45.

58. Zhang D, Tan T, Gao L, Zhao W, Wang P. Preparation of azithromycin nanosuspensions by high pressure homogenization and its physicochemical characteristics studies. Drug Dev Ind Pharm 2007;33:569-75.

59. Gao L, Zhang D, Chen M, Zheng T, Wang S. Preparation and characterization of an oridonin nanosuspension for solubility and dissolution velocity enhancement. Drug Dev Ind Pharm 2007;33:1332-9.

60. Jiang T, Han N, Zhao B, Xie Y, Wang S. Enhanced dissolution rate and oral bioavailability of simvastatin nanocrystal prepared by sonoprecipitation. Drug Dev Ind Pharm 2012;38:1230-9.

61. Peltonen L, Hirvonen J. Pharmaceutical nanocrystals by nanomilling: critical process parameters, particle fracturing and stabilization methods. J Pharm Pharmacol 2010;62:1569-79.

62. Van Eerdenbrugh B, Vermant J, Martens JA, Froyen L, Van Humbeeck J, Augustijns $\mathrm{P}$, et al. A screening study of surface stabilization during the production of drug nanocrystals. J Pharm Sci 2009;98:2091-103.

63. Sepassi S, Goodwin DJ, Drake AF, Holland S, Leonard G, Martini L, et al. Effect of polymer molecular weight on the production of drug nanoparticles. J Pharm Sci 2007;96:2655-66.

64. Choi JY, Yoo JY, Kwak HS, Uk Nam B, Lee J. Role of polymeric stabilizers for drug nanocrystal dispersions. Curr Appl Phys 2005;5:472-4.

65. Na G, Stevens J, Yuan B, Rajagopalan N. Physical stability of ethyl diatrizoate nanocrystalline suspension in steam sterilization. Pharm Res 1999;16:569-74. 
66. Annapragada A, Adjei A. Numerical simulation of milling processes as an aid to process design. Int J Pharm 1996;136:1-11.

67. Deng Z, Xu S, Li S. Understanding a relaxation behavior in a nanoparticle suspension for drug delivery applications. Int J Pharm 2008;351:236-43.

68. Zhang Y, Furyk S, Bergbreiter DE, Cremer PS. Specific ion effects on the water solubility of macromolecules: PNIPAM and the Hofmeister series. J Am Chem Soc 2005; 127:14505-10.

69. Nakano T, Yuasa H. Suppression of agglomeration in fluidized bed coating. IV. effects of sodium citrate concentration on the suppression of particle agglomeration and the physical properties of HPMC film. Int J Pharm 2001;215:3-12.

70. Williams HD, Ward R, Hardy IJ, Melia CD. The effect of sucrose and salts in combination on the drug release behaviour of an HPMC matrix. Eur J Pharm Biopharm 2010;76:433-6.

71. Ploehn HJ, Russel WB. Interactions between colloidal particles and soluble polymers. Adv Chem Eng1990;15:137-228.

72. Lee J, Lee SJ, Choi JY, Yoo JY, Ahn CH. Amphiphilic amino acid copolymers as stabilizers for the preparation of nanocrystal dispersion. Eur J Pharm Sci 2005;24:441-9.

73. Zhang Y, Cremer PS. Interactions between macromolecules and ions: the Hofmeister series. Current Opin Chem Biol 2006;10:658-63.

74. Van Eerdenbrugh B, Froyen L, Van Humbeeck J, Martens JA, Augustijns P, Van den Mooter G. Drying of crystalline drug nanosuspensions-the importance of surface hydrophobicity on dissolution behavior upon redispersion. Eur J Pharm Sci 2008;35:127-35.

75. Wang B, Zhang W, Zhang W, Mujumdar AS, Huang L. Progress in drying technology for nanomaterials. Dry Technol 2005;23:7-32.

76. Zhang GGZ, Gu C, Zell MT, Burkhardt RT, Munson EJ, Grant DJW. Crystallization and transitions of sulfamerazine polymorphs. J Pharm Sci 2002;91:1089-100.

77. Cocchetto DM, Levy G. Absorption of orally administered sodium sulfate in humans. J Pharm Sci 1981;70:331-3. 
78. Leonardi D, Barrera M, Lamas M, Salomón C. Development of Prednisone: Polyethylene glycol 6000 fast-release tablets from solid dispersions: solid-state characterization, dissolution behavior, and formulation parameters. AAPS PharmSciTech 2007;8:221-8.

79. Ning X, Sun J, Han X, Wu Y, Yan Z, Han J, He Z. Strategies to improve dissolution and oral absorption of glimerpiride tablets: solid dispersion versus micronization techniques. Drug Dev Ind Pharm 2011;37:727-36.

80. Palanisamy M, Khanam J. Solid dispersion of prednisolone: solid state characterization and improvement of dissolution profile. Drug Dev Ind Pharm 2011;37:373-86.

81. Lindfors L, Skantze P, Skantze U, Westergren J, Olsson U. Amorphous drug nanosuspensions 3. particle dissolution and crystal growth. Langmuir 2007;23:9866-74.

82. Frey FJ, Escher G, Frey BM. Pharmacology of 11ß-hydroxysteroid dehydrogenase. Steroids 1994;59:74-9.

83. Schuh S, Reisman J, Alshehri M, Dupuis A, Corey M, Arseneault R, et al. A comparison of inhaled fluticasone and oral prednisone for children with severe acute asthma. New Engl J Med 2000;343:689-94.

84. Kung FH, Nyhan WL, Cuttner J, Falkson G, Lanzkowsky P, Duca VD, et al. Vincristine, prednisone and L-asparaginase in the induction of remission in children with acute lymphoblastic leukemia following relapse. Cancer 1978;41:428-34.

85. Hecq J, Deleers M, Fanara D, Vranckx H, Amighi K. Preparation and characterization of nanocrystals for solubility and dissolution rate enhancement of nifedipine. Int J Pharm 2005;299:167-77.

86. Mauludin R, Müller RH, Keck CM. Development of an oral rutin nanocrystal formulation. Int J Pharm 2009;370:202-9.

87. Chiou WL, Riegelman S. Pharmaceutical applications of solid dispersion systems. J Pharm Sci 1971;60:1281-302.

88. Serajuddin ATM. Solid dispersion of poorly water-soluble drugs: early promises, subsequent problems, and recent breakthroughs. J Pharm Sci 1999;88:1058-66.

89. Vadher A, Parikh J, Parikh R, Solanki A. Preparation and characterization of coground mixtures of aceclofenac and neusilin us 2 for dissolution enhancement of aceclofenac. AAPS PharmSciTech 2009;10:606-14. 
90. Maclean J, Medina C, Daurio D, Alvarez-Nunez F, Jona J, Munson E, et al. Manufacture and performance evaluation of a stable amorphous complex of an acidic drug molecule and neusilin. J Pharm Sci 2011;100:3332-44.

91. Qian KK, Bogner RH. Application of mesoporous silicon dioxide and silicate in oral amorphous drug delivery systems. J Pharm Sci 2012;101:444-63.

92. Maciel GE. Probing hydrogen bonding and the local environment of silanols on silica surfaces via nuclear spin cross polarization dynamics. J Am Chem Soc 1996;118:401-6.

93. Chuang IS, Maciel GE. A detailed model of local structure and silanol hydrogen bonding of silica gel surfaces. J Phys Chem B 1997;101:3052-64.

94. Gupta MK, Vanwert A, Bogner RH. Formation of physically stable amorphous drugs by milling with neusilin. J Pharm Sci 2003;92:536-51.

95. Neusilin $®$ : the extraordinary excipient for oral solid dosage forms. Technical Newsletter. http://www.neusilin.com/multicms/neusilin/pdf/news/29/2 neusilin_n ewsletter nov07.pdf. Accessed 7 Nov 2007

96. Konno T, Kinuno K, Kataoka K. Physical and chemical changes of medicinals in mixtures with adsorbents in the solid state. I.: effect of vapor pressure of the medicinals on changes in crystalline properties. Chem Pharm Bull 1986;34:301-7.

97. Gupta P, Kakumanu VK, Bansal AK. Stability and solubility of celecoxib-PVP amorphous dispersions: a molecular perspective. Pharm Res 2004;21:1762-9.

98. Johansen H, Møller N. Solvent deposition method for enhancement of dissolution rate: Importance of drug-to-excipient ratio. J Pharm Sci 1978;67:134-6.

99. Law D, Schmitt EA, Marsh KC, Everitt EA, Wang W, Fort JJ, et al. RitonavirPEG 8000 amorphous solid dispersions: In vitro and in vivo evaluations. J Pharm Sci 2004;93:563-70.

100. Law D, Krill SL, Schmitt EA, Fort JJ, Qiu Y, Wang W, et al. Physicochemical considerations in the preparation of amorphous ritonavir-poly(ethylene glycol) 8000 solid dispersions. J Pharm Sci 2011;90:1015-25.

101. Middleton EJ, Davies JM, Morrison AB. Relationship between rate of dissolution, disintegration time, and physiological availability of riboflavin in sugar-coated tablets. J Pharm Sci 1964;53:1378-80. 
102. Albertini B, Cavallari C, Passerini N, Voinovich D, González-Rodrí guez ML, Magarotto L, et al. Characterization and taste-masking evaluation of acetaminophen granules: comparison between different preparation methods in a high-shear mixer. Eur J Pharm Sci 2004;21:295-303.

103. Kayumba PC, Huyghebaert N, Cordella C, Ntawukuliryayo JD, Vervaet C, Remon JP. Quinine sulphate pellets for flexible pediatric drug dosing: formulation development and evaluation of taste-masking efficiency using the electronic tongue. Eur J Pharm Biopharm 2007;66:460-5.

104. Andronis V, Yoshioka M, Zografi G. Effects of sorbed water on the crystallization of indomethacin from the amorphous state. J Pharm Sci 1997;86:346-51.

105. Morgan DJ, Morgan A. Studies on the retention and metabolism of inhaled methyl iodide-I: retention of inhaled methyl iodide. Health Physics 1967;13:105565.

106. Risher JF, Keith LS. Iodine and inorganic iodides: human health aspects, $1^{\text {st }}$ ed., Geneva: WHO Press; 2009;14-6.

107. Dai G, Levy O, Carrasco N. Cloning and characterization of the thyroid iodide transporter. Nature 1996;379:458-60.

108. Smanik PA, Ryu KY, Theil KS, Mazzaferri EL, Jhiang SM. Expression, exonintron organization, and chromosome mapping of the human sodium iodide symporter. Endocrinology 1997;138:3555-8.

109. Delange F. The disorders induced by iodine deficiency. Thyroid 1994;4:107-28.

110. Blum M, Eisenbud M. Reduction of thyroid irradiation from 131I by potassium iodide. J Am Med Assoc 1967;200:1036-40.

111. Zanzonico PB, Becker DV. Effects of time of administration and dietary iodine levels on potassium iodide (KI) blockade of thyroid irradiation by $131 \mathrm{I}$ from radioactive fallout. Health Physics 2000;78:660-7.

112. McNeill SC, Potts RO, Francoeur ML. Local enhanced topical delivery (LETD) of drugs: Does it truly exist? Pharm Res 1992;9:1422-7.

113. Wallace SM, Barnett G. Pharmacokinetic analysis of percutaneous absorption; evidence of parallel penetration pathways for methotrexate. J Pharmacokinet Pharm 1978;6:315-25.

114. Keister JC, Kasting GB. The use of transient diffusion to investigate transport pathways through skin. J Control Release 1986;4:111-7. 
115. Edwards DA, Langer R. A linear theory of transdermal transport phenomena. J Pharm Sci 1994;83:1315-34.

116. Pechtold LARM, Abraham W, Potts RO. The influence of an electric field on ion and water accessibility to stratum corneum lipid lamellae. Pharm Res 1996;13:1168-73.

117. Boddé HE, Kruithof MAM, Brussee J, Koerten HK. Visualisation of normal and enhanced $\mathrm{HgCl} 2$ transport through human skin in vitro. Int J Pharm 1989;53:1324.

118. Cornwell PA, Barry BW. The routes of penetration of ions and 5-fluorouracil across human skin and the mechanisms of action of terpene skin penetration enhancers. Int J Pharm 1993;94:189-94.

119. Costigan SC, Booth PJ, Templer RH. Estimations of lipid bilayer geometry in fluid lamellar phases. Biochimica et Biophysica Acta (BBA) - Biomembranes. 2000;1468:41-54.

120. Mitragotri S. Modeling skin permeability to hydrophilic and hydrophobic solutes based on four permeation pathways. J Control Release 2003;86:69-92.

121. Zhang K, Chen M, Scriba GKE, Abraham MH, Fahr A, Liu X. Human skin permeation of neutral species and ionic species: Extended linear free-energy relationship analyses. J Pharm Sci 2012;101:2034-44.

122. Lawrence MJ, Rees GD. Microemulsion-based media as novel drug delivery systems. Adv Drug Deliv Rev 2000;45:89-121.

123. Hathout RM, Woodman TJ, Mansour S, Mortada ND, Geneidi AS, Guy RH. Microemulsion formulations for the transdermal delivery of testosterone. Eur J Pharm Sci 2010;40:188-96.

124. Monzer F. Phase behavior, transport, diffusion and structural parameters of nonionic surfactants microemulsions. J Mol Liq 2008;139:14-22.

125. Lee PJ, Ahmad N, Langer R, Mitragotri S, Prasad Shastri V. Evaluation of chemical enhancers in the transdermal delivery of lidocaine. Int J Pharm 2006;308:33-9.

126. Jaeger J, Sorensen K, Wolff SP. Peroxide accumulation in detergents. J Biochem Biophys Methods 1994;29:77-81.

127. Lever M. Peroxides in detergents as interfering factors in biochemical analysis. Anal Biochem 1997;83:274-84. 
128. Mancuso JR, McClements DJ, Decker EA. Ability of iron to promote surfactant peroxide decomposition and oxidize $\alpha$-tocopherol. J Agric Food Chem 1999;47:4146-9.

129. López A, Llinares F, Cortell C, Herráez M. Comparative enhancer effects of Span ${ }^{\circledR} 20$ with Tween $₫ 20$ and Azone ${ }^{\circledR}$ on the in vitro percutaneous penetration of compounds with different lipophilicities. Int J Pharm 2000;202:133-40.

130. Azeem A, Ahmad F, Khar R, Talegaonkar S. Nanocarrier for the transdermal delivery of an antiparkinsonian drug. AAPS PharmSciTech 2009;10:1093-103.

131. Singh A, Chaurasiya A, Singh M, Upadhyay S, Mukherjee R, Khar R. Exemestane loaded self-microemulsifying drug delivery system (SMEDDS): development and optimization. AAPS PharmSciTech 2008;9:628-34.

132. Gwak HS, Chun IK. Effect of vehicles and penetration enhancers on the in vitro percutaneous absorption of tenoxicam through hairless mouse skin. Int J Pharm 2002;236:57-64.

133. Kabalnov A, Olsson U, Wennerstroem H. Salt effects on nonionic microemulsions are driven by adsorption/depletion at the surfactant monolayer. $\mathrm{J}$ Phys Chem 1995;99:6220-30.

134. Zhang Y, Cremer PS. Interactions between macromolecules and ions: the Hofmeister series. Curr Opin Chem Bio 2006;10:658-63.

135. Yuan JS, Ansari M, Samaan M, Acosta EJ. Linker-based lecithin microemulsions for transdermal delivery of lidocaine. Int J Pharm 2008;349:130-43.

136. Djordjevic L, Primorac M, Stupar M, Krajisnik D. Characterization of caprylocaproyl macrogolglycerides based microemulsion drug delivery vehicles for an amphiphilic drug. Int J Pharm 2004;271:11-9.

137. Gradzielskin M, Hoffmann H. Rheological properties of microemulsions. In: Kumar P, and Mittal KL (ed.) Handbook of Micromulsion Science and Technology, $1^{\text {st }}$ ed., New York: Marcel Dekker Inc. 1999;357-86.

138. Li X, He G, Zheng W, Xiao G. Study on conductivity property and microstructure of TritonX-100/alkanol/n-heptane/water microemulsion. Colloid Surface A 2010;360:150-8.

139. Mathew C, Patanjali PK, Nabi A, Maitra A. On the concept of percolative conduction in water-in-oil microemulsions. Colloid Surface 1998;30:253-63. 
140. Jian X, Ganzuo L, Zhiqiang Z, Guowei Z, Kejian J. A study of the microstructure of CTAB/1-butanol/octane/water system by PGSE-NMR, conductivity and cryoTEM. Colloid Surface A 2001;191:269-78.

141. Eicke HF, Borkovec M, Das-Gupta B. Conductivity of water-in-oil microemulsions: a quantitative charge fluctuation model. J Phys Chem 1989;93:314-7.

142. Yuan Y, Li SM, Mo FK, Zhong DF. Investigation of microemulsion system for transdermal delivery of meloxicam. Int J Pharm 2006;321:117-23.

143. Jain J, Fernandes C, Patravale V. Formulation development of parenteral phospholipid-based microemulsion of Etoposide. AAPS PharmSciTech 2010;11:826-31.

144. De Gennes PG, Taupin C. Microemulsions and the flexibility of oil/water interfaces. J Phys Chem 1982;86:2294-304 


\section{VITA}

Hao Lou was born in Jinhua, Zhejiang, China, in 1987. He started his undergraduate education at Zhejiang University, Hangzhou, China. Afterwards, Hao transferred to Purdue University, West Lafayette, IN, where he graduated with a Bachelor degree in Pharmaceutical Sciences (Minor in Statistics) in May, 2010. In the Fall of 2010, he joined the graduate program in the department of Pharmaceutical Sciences, the University of Tennessee Health Science Center, Memphis, from where he will obtain his MS in Pharmaceutical Sciences in May 2013. 